\title{
Comparing variants of strategic ability: how uncertainty and memory influence general properties of games
}

\author{
Nils Bulling · Wojciech Jamroga
}

Published online: 17 July 2013

(C) The Author(s) 2013

\begin{abstract}
Alternating-time temporal logic (ATL) is a modal logic that allows to reason about agents' abilities in game-like scenarios. Semantic variants of ATL are usually built upon different assumptions about the kind of game that is played, including capabilities of agents (perfect vs. imperfect information, perfect vs. imperfect memory, etc.). ATL has been studied extensively in previous years; however, most of the research focused on model checking. Studies of other decision problems (e.g., satisfiability) and formal meta-properties of the logic (like axiomatization or expressivity) have been relatively scarce, and mostly limited to the basic variant of ATL where agents possess perfect information and perfect memory. In particular, a comparison between different semantic variants of the logic is largely left untouched. In this paper, we show that different semantics of ability in ATL give rise to different validity sets. The issue is important for several reasons. First, many logicians identify a logic with its set of true sentences. As a consequence, we prove that different notions of ability induce different strategic logics. Secondly, we show that different concepts of ability induce different general properties of games. Thirdly, the study can be seen as the first systematic step towards satisfiability-checking algorithms for ATL with imperfect information. We introduce sophisticated unfoldings of models and prove invariance results that are an important technical contribution to formal analysis of strategic logics.
\end{abstract}

Keywords Alternating-time temporal logic · Validity and satisfiability · Properties of games · Games with imperfect information

\footnotetext{
N. Bulling $(\varangle)$

Department of Informatics, Clausthal University of Technology, Clausthal-Zellerfeld, Germany e-mail: bulling@in.tu-clausthal.de

\section{W. Jamroga}

Computer Science and Communication Research Unit, and Interdisciplinary Centre on Security, Reliability and Trust, University of Luxembourg, Luxembourg Luxembourg

e-mail: wojtek.jamroga@uni.lu
} 


\section{Introduction}

Alternating-time temporal logic (ATL) [7,9] is a temporal logic that incorporates some basic game theoretic notions. In ATL we can for instance express that a group of agents is able to bring about $\varphi$, i.e., the agents in the group are able to enforce that property $\varphi$ holds whatever the other agents might do. Semantic variants of ATL are usually derived from different assumptions about agents' capabilities. Can the agents "see" the current state of the system, or only a part of it? Can they memorize the whole history of observations in the game? Is it enough that they have a way of enforcing the required temporal property "objectively", or should they be able to come up with the right strategy on their own? Different answers to these questions induce different semantics of strategic ability, and they clearly give rise to different analyses of a given game model. However, it is not entirely clear to what extent they give rise to different logics. One natural question that arises in this respect is whether these semantic variants generate different sets of valid (and, dually, satisfiable) sentences. In this paper, we settle the issue and show that most "classical" semantic variants of ATL are indeed different, and we characterize the relationship between their sets of validities.

The question is important for several reasons. First, many logicians identify a logic with the set of sentences that are true in the logic; a semantics is just a possible way of defining the set, alternative to an axiomatic inference system. Thus, by comparing validity sets we compare the respective logics in the traditional sense. Secondly, validities of ATL capture general properties of games under consideration: if, e.g., two variants of ATL generate the same valid sentences then the underlying notions of ability induce the same kind of games. All the variants studied here are defined over the same class of models (imperfect information concurrent game structures) that generalizes extensive form games. The difference between games "induced" by different semantics lies in the available strategies and the winning conditions for them.

Thirdly, the satisfiability problem for ATL, though far less studied than model checking, is not necessarily less important. While model checking ATL can be seen as the logical analogue of game solving, satisfiability corresponds naturally to mechanism design. A systematic study on the abstract level is the first step towards algorithms that solve the problem.

Our results are relevant also outside the logical context. As already mentioned, by looking at validity sets we study general properties of strategic ability under various semantic assumptions. Ultimately, we show that what agents can achieve is more sensitive to the strategic model of an agent (and a precise notion of achievement) than it was generally realized. No less importantly, our study reveals that some natural properties-usually taken for granted when reasoning about temporal evolution of systems-may cease to be universally true if we change the strategic setting. Examples include fixpoint characterizations of temporal/strategic operators (that enable incremental synthesis and iterative execution of strategies) and the duality between necessary and obtainable outcomes in a game. The former kind of properties is especially important for practical purposes, since fixpoint equivalences provide the basis for most model checking and satisfiability checking algorithms. Finally, we introduce sophisticated unfoldings of models to show invariance results with respect to memoryless and perfect recall strategies. The unfoldings form an important technical contribution of this article. We believe that their impact goes beyond ATL, as they can probably be applied to other strategy logics. For example, it would be interesting to see which unfoldings preserve the truth values of formulae when imperfect information is combined with strategic commitment [66], or when explicit quantification over strategies is allowed [20,50,51].

The paper is structured as follows. We begin by presenting the relevant variants of ATL in Sects. 2 and 3. Then we define several unfoldings of ATL models, and show that they 
preserve truth of ATL formulae under appropriate assumptions in Sect. 4. This is the most technical part of the paper, and readers interested only in the main conceptual contribution are advised to skip it and proceed to the next section. In Sect. 5, we show the formal relationships between validity sets for different variants of ATL. Summary of the main results and some conclusions are presented in Sect. 6.

About this article Preliminary versions of this paper appeared in $[40,41]$. The journal version adds proofs, new results, examples, and more extensive discussions. This applies in particular to Sect. 4 where we stress the importance of tree-like unfoldings and provide a sophisticated construction as well as full proofs. We have also extended the results from [40,41] (formulated mainly for the restricted language of ATL) to the more general language of ATL*.

\subsection{Related work}

ATL has been studied extensively in the last 15 years. The research can be roughly divided into the computational and conceptual strands. The former has been focused on the way in which ATL and its extensions can be used for verification of multi-agent systems, in particular what is the complexity of model checking, and how one can overcome the inherent difficulties. An interested reader is referred to [13] for an overview, and to [9,17,20,24,42, $48,57,59]$ for more specific results; some attempts at taming the complexity were proposed e.g. in $[18,23,38,46,47]$. Studies on other decision problems than model checking were much less frequent, though satisfiability of the basic variant of ATL has been investigated in $[30,31,54,64]$.

The conceptual strand originally emerged in quest of the "right" semantics for strategies under uncertainty. ATL was combined with epistemic logic [1,2,39,60-62], and several semantic variants were defined that match various possible interpretations of ability [35,39,43,45,57]. Moreover, many conceptual extensions have been considered, e.g., with explicit reasoning about strategies, rationality assumptions and solution concepts $[19,20,58,63,65]$, agents with bounded resources $[5,6,14,15]$, coalition formation and negotiation [12], opponent modeling and action in stochastic environments $[16,37,55,56]$ and reasoning about irrevocable plans and interplay between strategies of different agents $[3,11]$.

In the rich literature on the conceptual virtues of alternating-time temporal logic, formal analysis is relatively scarce. Axiomatization of the basic variant of ATL was proposed in [31], and its expressivity was addressed in [9,48]. Axiomatization of a particular variant of imperfect information was proposed in [32]. For comparative studies, invariance of the basic semantics with respect to a couple of classes of models was proven in [27], and the correspondence between abstract and concrete models of strategic logics was the object of study in $[29,28,52]$. Surprisingly, relationships between the "classical" semantic variants of ATL (as defined e.g. in [57]) have not yet been studied, though analogous results exist that compare more sophisticated variations to a more standard variant (cf. [36] for undominated play, [3] for irrevocable strategies, [4] for agents with bounded memory, and [18] for recomputable strategies under uncertainty). That means in particular that formal properties of strategic ability under imperfect information are largely left untouched. We are trying to fill in the gap, and start a more systematic charting of the landscape.

\section{Reasoning about strategic abilities}

Alternating-time temporal logic ATL [7,9] is a temporal logic that incorporates some basic game-theoretical notions. Essentially, ATL generalizes the branching time logic CTL [21] by 
replacing path quantifiers $\mathrm{E}, \mathrm{A}$ with strategic modalities $\langle\langle A\rangle\rangle$. Informally, $\langle\langle A\rangle\rangle \gamma$ expresses that the group of agents $A$ has a collective strategy to enforce temporal property $\gamma$. ATL formulae include temporal operators: " $\bigcirc$ " ("in the next state"), " $\square$ " ("always from now on"), " $\vee$ " ("now or sometime in the future"), and $\mathcal{U}$ ("until"). Since ATL offers no way of representing agents' uncertainty in its models, and no operators to refer to agents' (lack of) knowledge in the object language, it allows to reason only about abilities of agents with perfect information about the current global state of the system.

\subsection{Syntax of ATL}

In the rest of the paper we assume that $\Pi$ is a nonempty set of proposition symbols and $\mathbb{A g t}$ a nonempty and finite set of agents. Alternating-time temporal logic comes in several syntactic variants, of which $\mathbf{A T L}^{*}$ is the broadest.

Definition 1 (Language of $\boldsymbol{A T L}^{*}$ ) The language of $\mathbf{A T L}^{*}$ is given by formulae $\varphi$ generated by the grammar below, where $A \subseteq \mathbb{A g t}$ is a set of agents, and $p \in \Pi$ is an atomic proposition:

$$
\begin{aligned}
& \varphi::=\mathrm{p}|\neg \varphi| \varphi \wedge \varphi \mid\langle\langle A\rangle \gamma, \\
& \gamma::=\varphi|\neg \gamma| \gamma \wedge \gamma|\bigcirc \gamma| \gamma \mathcal{U} \gamma .
\end{aligned}
$$

The "sometime" and "always" operators can be defined as $\diamond \gamma \equiv \top \mathcal{U} \gamma$ and $\square \gamma \equiv \neg \diamond \neg \gamma$.

Formulae $\varphi$ are called state formulae, and $\gamma$ path formulae of ATL*. A path formula is simple if it consists of a temporal operator followed immediately by a state subformula and in the case of "until" the operator is also immediately preceded by a state subformula. In other words, temporal operators have to be applied to state subformulae.

The best known syntactic variant of alternating time temporal logic is ATL in which every occurrence of a strategic modality is immediately coupled with a temporal operator, i.e., we have coupled operators of the form $\langle\langle A\rangle\rangle,\langle\langle A\rangle\rangle$, , and $\langle\langle A\rangle\rangle \mathcal{U}$. The language of $\mathbf{A T L}^{+}$ sits between ATL* and ATL: it allows strategic modalities to be followed by a Boolean combination of simple temporal subformulae.

Formally, formulae of ATL are defined be the following grammar: ${ }^{1}$

$$
\varphi::=\mathrm{p}|\neg \varphi| \varphi \wedge \varphi|\langle A\rangle\rangle \bigcirc \mid\langle\langle A\rangle \square \varphi|\langle\langle A\rangle\rangle \mathcal{U} \varphi
$$

and $\mathbf{A} \mathbf{T L}{ }^{+}$formulae by:

$$
\varphi::=\mathrm{p}|\neg \varphi| \varphi \wedge \varphi|\langle A\rangle\rangle \gamma, \quad \gamma::=\neg \gamma|\gamma \wedge \gamma| \bigcirc \varphi \mid \varphi \mathcal{U} \varphi .
$$

Example 1 The ATL formula 《jamesbond, octopussy》〉 $\diamond$ iss says that James Bond and Octopussy can eventually kiss, no matter how the other agents act. On the other hand, $\langle\langle$ jamesbond, jaws》 $(\square \neg$ crash $\wedge \diamond$ land) (James Bond and Jaws can prevent the space ship from crashing and make it eventually land) is a formula of $\mathbf{A T L} \mathbf{L}^{+}$but not ATL. Finally, $\langle\langle$ jamesbond $\rangle\rangle \square \diamond$ deadBlofeld $\wedge \neg\langle\langle$ jamesbond $\rangle\rangle \square$ deadBlofeld is an ATL Afor- $^{*}$ mula (which clearly belongs to neither ATL nor $\mathbf{A T L}^{+}$) which states that agent 007 can kill Ernst Stavro Blofeld infinitely many times, but he cannot kill Blofeld once and forever.

\subsection{Basic models of ATL}

In [9], the semantics of alternating-time temporal logic is defined over a variant of transition systems where transitions are labeled with combinations of actions, one per agent.

1 Note that "always" is not definable from "until" in ATL [48], and has to be added explicitly to the language. 
Fig. 1 Two robots and a carriage: concurrent game structure $M_{0}$

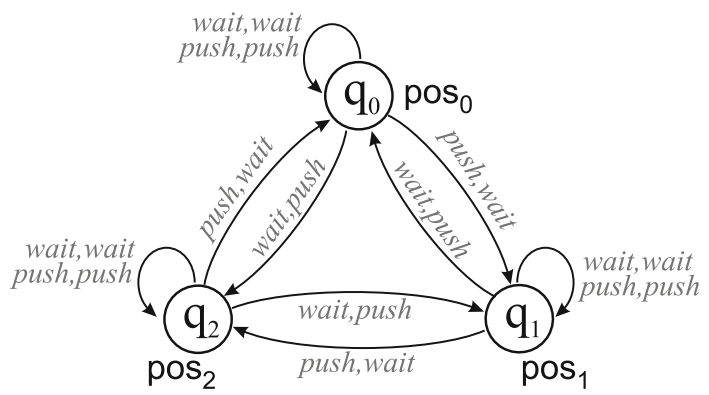

Definition 2 (Concurrent game structure) A concurrent game structure ${ }^{2}$ (CGS) is a tuple $\mathfrak{M}=\langle\mathbb{A g t}, S t, \Pi, \pi, A c t, d, o\rangle$ which includes a nonempty finite set of all agents $\mathbb{A g t}=$ $\{1, \ldots, k\}$, a nonempty (possibly infinite) set of states $S t$, a set of atomic propositions $\Pi$ and their valuation $\pi: \Pi \rightarrow 2^{S t}$, and a nonempty set of (atomic) actions Act. Function $d: \mathbb{A g t} \times S t \rightarrow 2^{A c t}$ defines nonempty sets of actions available to agents at each state, and $o$ is a (deterministic) transition function that assigns the outcome state $q^{\prime}=o\left(q, \alpha_{1}, \ldots, \alpha_{k}\right)$ to state $q$ and a tuple of actions $\alpha_{i} \in d(i, q)$ that can be executed by $\mathbb{A g t}$ in $q$.

Thus, we assume that all the agents execute their actions synchronously; the combination of the actions, together with the current state, determines the next transition of the system.

In the rest of the paper, we will write $d_{i}(q)$ instead of $d(i, q)$, and we will denote the set of collective choice of group $A$ at state $q$ by $d_{A}(q)=\prod_{i \in A} d_{i}(q)$.

We will sometimes use the term pointed CGS for a pair $(\mathfrak{M}, q)$ of a concurrent game structure and a state in it.

Definition 3 (Path) A path $\lambda=q_{0} q_{1} q_{2} \ldots$ is an infinite sequence of states such that there is a transition between each $q_{i}, q_{i+1}$. We use $\lambda[i]$ to denote the $i$ th position on path $\lambda$ (starting from $i=0)$ and $\lambda[i, \infty]$ to denote the subpath of $\lambda$ starting from $i$. The set of paths starting in $q$ is denoted by $\Lambda_{\mathfrak{M}}(q)$.

Example 2 (Robots and Carriage) Consider the scenario depicted in Fig. 1. Two robots push a carriage from opposite sides. As a result, the carriage can move clockwise or anticlockwise, or it can remain in the same place. We assume that each robot can either push (action push) or refrain from pushing (action wait). Moreover, they both use the same force when pushing. Thus, if the robots push simultaneously or wait simultaneously, the carriage does not move. When only one of the robots is pushing, the carriage moves accordingly.

To make our model of the domain discrete, we identify three different positions of the carriage, and associate them with states $q_{0}, q_{1}$, and $q_{2}$. We label the states with propositions $\operatorname{pos}_{0}, \operatorname{pos}_{1}, \operatorname{pos}_{2}$, respectively, to allow for referring to the current position of the carriage in the object language.

\subsection{Finite versus infinite CGS}

In our definition of CGS (Def. 2.2) we have not put up any requirement of finiteness with respect to the set of states and actions. The only requirement is that the set of agents must be

2 We would like to note that it is essential for this work that we do not require a finite set of states or actions. We give a more detailed discussion in Sect. 2.3. 
finite. In particular, we allow for infinitely many states in a model; we also allow for infinitely branching models. In this section we shall discuss this choice in more detail.

We begin by reviewing the literature and showing that both types of CGS-finite and infinite ones-have been considered by other authors. The semantics of ATL in concurrent game models was originally proposed for finite structures only [9]. ${ }^{3}$ Many follow-up papers also adopted the assumption of finite models, for example [43,57] that studied variants of ATL with imperfect information [11], which extended ATL with persistent strategies, in [48] the expressive power of ATL is investigated, etc.

On the other hand, other authors did not restrict their analysis to the finite case, beginning with the work on coalition logic [52,53], through comparative studies of different semantics of ATL [26,27], the interplay between knowledge and strategies [2,39], strategic play in the presence of intentions and commitment $[3,44]$, and so on. Also, different formalisms extending ATL* with explicit quantification over strategies follow different assumptions: on one hand, the strategy logic by Chatterjee et al. [20] assumes models to be finite; on the other, the strategy logic recently proposed by Mogavero et al. [51] only requires states and actions to be countable.

As we have already stated, we assume neither St nor Act to be finite (or even enumerable). How does that affect our work? First of all, for the new results in this article, it is especially important that some existing technical results can be applied to infinite models. This concerns in particular the axiomatization of ATL from [31] which was shown sound and complete for finite as well as infinite concurrent game models. To be more precise, the authors of [31] allow for infinitely many states, but assume that, at any state, there are only finally many outgoing transitions. However, their results extend to the case of infinite branching in a straightforward way. We use the axiomatization as a source of "standard" validities (like the fixpoint characterization for $\langle\langle A\rangle\rangle \diamond)$, and to show that the semantics of "perfect information memoryless ATL" and "perfect information perfect recall ATL" coincide also for infinite models (Proposition 1). Moreover, the notion of model equivalence for ATL (alternating bisimulation alias strategic bisimulation), while originally proposed for finite models only [10], was extended to the unrestricted case and proved correct in [3]. We use and extend the concept to prove invariance results for tree-like unfoldings in Sect. 4.

Secondly, all the results proposed in this paper are proved to hold if the semantics of $\boldsymbol{A T L}$ and $\boldsymbol{A T L}^{*}$ does not restrict the class of models to finite ones. More precisely, it may be possible that one of our inclusion results between the validity sets of two logics, $\operatorname{Val}\left(\mathrm{L}_{1}\right) \subseteq \operatorname{Val}\left(\mathrm{L}_{2}\right)$, requires the existence of an infinite model. This does not mean that the theorems that we present do not hold in the class of finite models. The latter issue, albeit interesting, is outside of the scope of the paper. Essentially, showing that our results hold in the finite semantics would require establishing finite model properties for the logics that we consider. To the best of our knowledge, such properties have only been proven for the "perfect information/perfect recall" variant of ATL [31] and ATL* [54]. Proving (or disproving) the finite model property for the other variants of ATL/ATL* is undoubtedly important, and we would like to study it further in the future. ${ }^{4}$

In summary:

1. our inclusion results rely on the fact that we define the semantics of ATL and ATL* in both finite and infinite models; and

\footnotetext{
${ }^{3}$ An interested reader may observe that the preliminary versions of the semantics (in alternating transition systems) did not assume models explicitly to be finite $[7,8]$. However, the authors de facto considered only finite models since they were solely interested in the model checking problem, where the input must be finite.

${ }^{4}$ We thank an anonymous JAAMAS reviewer for suggesting this.
} 
2. whenever a finite model property holds for two logics under consideration, our results comparing the two logics apply also when the semantics is restricted to finite models.

\subsection{Strategies and abilities in basic semantics of ATL}

ATL modalities refer to the outcome of strategic play for a given coalition. Following the tradition of extensive form games in game theory, a strategy of agent $a$ is understood as a plan that specifies what $a$ is going to do in each situation. In the standard version of ATL [7,9], strategies are represented by functions $s_{a}: S t^{+} \rightarrow A c t$. Thus, it is implicitly assumed that agents have perfect information (at each moment, they can precisely recognize the current global state of the system) and perfect recall (they can base their choices on the whole history of the game so far, not just the last state). Alternatively, one can assume that agents have no memory beyond what is already contained in the current state, which gives rise to the notion of memoryless (or positional) strategy. As we explain more systematically in Sect. 3, we will use the notation from [57] where $i$ (resp. I) stands for imperfect (resp. perfect) information, and $r$ (resp. $R$ ) for imperfect (resp. perfect) recall.

Definition 4 (IR- and Ir-strategies) Let $\mathfrak{M}$ be a CGS over states St and actions Act. A perfect information perfect recall strategy for agent $a$ in $\mathfrak{M}$ (IR-strategy in short) is a function $s_{a}: S t^{+} \rightarrow$ Act such that $s_{a}\left(q_{0} q_{1} \ldots q_{n}\right) \in d_{a}\left(q_{n}\right)$. The set of such strategies is denoted by $\Sigma_{a}^{I R}$.

A perfect information memoryless strategy for agent $a$ in $\mathfrak{M}$ (Ir -strategy in short) is a function $s_{a}: S t \rightarrow$ Act where $s_{a}(q) \in d_{a}(q)$. The set of such strategies is denoted by $\Sigma_{a}^{I r}$.

A collective strategy for a group of agents $A=\left\{a_{1}, \ldots, a_{r}\right\}$ is simply a tuple of individual strategies $s_{A}=\left\langle s_{a_{1}}, \ldots, s_{a_{r}}\right\rangle$. The set of such strategies is denoted by $\Sigma_{A}^{I R}$ (for $I R$ strategies) and $\Sigma_{A}^{I r}$ (for $I r$ strategies, respectively). ${ }^{5}$

The "outcome" function out $\left(q, s_{A}\right)$ returns the set of all paths that may occur when agents $A$ execute strategy $s_{A}$ from state $q$ onward. Let $a \in A$; by $\left.s_{A}\right|_{a}$, we denote agent $a$ 's part $s_{a}$ of the collective strategy $s_{A}$.

Definition 5 (Outcome) The outcome out $t_{\mathfrak{M}}\left(q, s_{A}\right)$ of an IR -strategy $s_{A}$ from state $q$ in model $\mathfrak{M}$ is the set of all paths $\lambda=q_{0} q_{1} q_{2} \ldots$ such that $q_{0}=q$ and for each $i=1,2, \ldots$ there exists a tuple of agents' decisions $\left\langle\alpha_{a_{1}}^{i-1}, \ldots, \alpha_{a_{k}}^{i-1}\right\rangle$ such that: $(i) \alpha_{a}^{i-1} \in d_{a}\left(q_{i-1}\right)$ for every $a \in \mathbb{A g t},(i i) \alpha_{a}^{i-1}=\left.s_{A}\right|_{a}\left(q_{0} q_{1} \ldots q_{i-1}\right)$ for every $a \in A$, and (iii) $o\left(q_{i-1}, \alpha_{a_{1}}^{i-1}, \ldots, \alpha_{a_{k}}^{i-1}\right)$ $=q_{i}$.

The outcome out $\mathfrak{M}\left(q, s_{A}\right)$ of an $\boldsymbol{I r}$-strategy $s_{A}$ is defined analogously but $\left.s_{A}\right|_{a}\left(q_{0} q_{1} \ldots\right.$ $\left.q_{i-1}\right)$ is replaced by $\left.s_{A}\right|_{a}\left(q_{i-1}\right)$.

Often, we will omit the subscript " $\mathfrak{M}$ " if it is clear from the context.

Let $\mathfrak{M}$ be a CGS, $q$ a state, and $\lambda$ a path in $\mathfrak{M}$. Now, the semantics of ATL* and its sublanguages can be defined by the following clauses [9]:

$$
\begin{aligned}
& \mathfrak{M}, q \models p \text { iff } q \in \pi(\mathrm{p}), \text { for } \mathrm{p} \in \Pi ; \\
& \mathfrak{M}, q \models \neg \varphi \text { iff } \mathfrak{M}, q \not \models ; \\
& \mathfrak{M}, q \models \varphi_{1} \wedge \varphi_{2} \text { iff } \mathfrak{M}, q \models \varphi_{1} \text { and } \mathfrak{M}, q \models \varphi_{2} ;
\end{aligned}
$$

\footnotetext{
5 As commonly done we assume an implicit order on the elements in $\mathbb{A g t}$ allowing to conveniently represent collective strategies as tuples. In our setting where agents are represented by natural numbers the order is apparent. In the general case, a collective strategy for $A$ is a function that associates individual strategies to the agents in $A$.
} 
$\mathfrak{M}, q \models\left\langle\langle A\rangle \gamma \quad\right.$ iff there is an $I R$-strategy $s_{A}$ for agents $A$ such that for each path $\lambda \in \operatorname{out}\left(q, s_{A}\right)$, we have $\mathfrak{M}, \lambda \models \gamma$.

$\mathfrak{M}, \lambda \models \varphi$ iff $\mathfrak{M}, \lambda[0] \models \varphi$;

$\mathfrak{M}, \lambda \models \neg \gamma$ iff $\mathfrak{M}, \lambda \not \models \gamma$

$\mathfrak{M}, \lambda \models \gamma_{1} \wedge \gamma_{2}$ iff $\mathfrak{M}, \lambda \models \gamma_{1}$ and $\mathfrak{M}, \lambda \models \gamma_{2}$;

$\mathfrak{M}, \lambda \models \bigcirc \gamma$ iff $\mathfrak{M}, \lambda[1, \infty] \models \gamma$; and

$\mathfrak{M}, \lambda \models \gamma_{1} \mathcal{U} \gamma_{2}$ iff there is an $i \in \mathbb{N}_{0}$ such that $\mathfrak{M}, \lambda[i, \infty] \models \gamma_{2}$ and $\mathfrak{M}, \lambda[j, \infty] \models \gamma_{1}$ for all $0 \leq j<i$.

Example 3 (Robots and Carriage, ctd.) Since the outcome of each robot's action depends on the current action of the other robot, no robot can make sure that the carriage moves to any particular position. So, we have for example that $M_{0}, q_{0} \models \neg\left\langle\langle 1\rangle \diamond\right.$ pos $_{1}$. On the other hand, the robots can cooperate to move the carriage. For instance, it holds that $M_{0}, q_{0} \models\langle\langle 1,2\rangle\rangle$ pos $_{1}$ (example strategy: robot 1 always pushes and robot 2 always waits). Moreover, single robots can play strategically to avoid the carriage entering a particular position: $M_{0}, q_{0} \models\left\langle\langle 1\rangle \square \neg \operatorname{pos}_{1}\right.$ (the strategy: wait in $q_{0}$ and push in $q_{2}$ ).

Note that the semantics does not address the issue of coordination between members of the coalition [25,34]: if there exist several successful joint strategies for $A$, the agents in $A$ are assumed to somehow choose between them.

Finally, validity and satisfiability in ATL are defined in the standard way.

Definition 6 (Validity and satisfiability) Formula $\varphi$ is valid in model $\mathfrak{M}$ iff it holds in every state of $\mathfrak{M}$, i.e., $\mathfrak{M}, q \models \varphi$ for every $q \in S t_{\mathfrak{M}}$. The formula is valid in a class of models $\mathcal{C}$ iff it is valid in every model from $\mathcal{C}$.

Dually, $\varphi$ is satisfiable in a class of models $\mathcal{C}$ iff there exists $\mathfrak{M} \in \mathcal{C}$ and a state $q$ in $\mathfrak{M}$ such that $\mathfrak{M}, q \models \varphi$.

\subsection{Some important validites}

We recall that the following fixpoint properties are valid in the basic semantics of ATL presented in Sect. 2.4:

$$
\begin{aligned}
\langle\langle A\rangle\rangle \square \varphi & \leftrightarrow \varphi \wedge\langle\langle A\rangle\rangle \bigcirc\langle\langle A\rangle \square \varphi \\
\langle\langle A\rangle\rangle \vee \varphi & \leftrightarrow \varphi \vee\langle\langle A\rangle\rangle\langle\langle A\rangle\rangle \nabla \varphi \\
\langle\langle A\rangle\rangle \varphi_{1} \mathcal{U} \varphi_{2} & \leftrightarrow \varphi_{2} \vee \varphi_{1} \wedge\left\langle\langle A \rangle \bigcirc \left\langle\langle A\rangle \varphi_{1} \mathcal{U} \varphi_{2} .\right.\right.
\end{aligned}
$$

Validity of these formulae was demonstrated in [9] for finite models, and in [31] for finitely branching models (with possibly infinitely many states). It is easy to check that the argument extends to models with infinite branching.

The intuitive meaning of the fixpoint equivalences is that planning for a long-term goal (like achieving $\varphi$ eventually, or maintaining $\varphi$ persistently) can be decomposed into finding a good opening move and a suitable follow-up. Such properties are crucial for building model checking and satisfiability checking algorithms, and in particular they allow for incremental iterative synthesis of strategies.

Moreover, the path quantifiers A, E of CTL can be expressed in the standard semantics of ATL with $\langle\langle\emptyset\rangle\rangle,\langle\langle\mathbb{A g t}\rangle\rangle$ respectively. Again, checking this is routine, even for models with infinitely many states and infinite branching. As a consequence, the CTL duality axioms can 
be rewritten in ATL, and become validities in the basic semantics:

$$
\begin{aligned}
\neg\langle\langle\mathrm{Agt}\rangle\rangle \bigcirc \varphi & \leftrightarrow\langle\langle\emptyset\rangle \bigcirc \neg \neg \varphi \\
\neg\langle\langle\mathrm{Agt}\rangle\rangle \nabla \varphi & \leftrightarrow\langle\langle\emptyset\rangle\rangle \square \neg \varphi, \\
\neg\langle\langle\emptyset\rangle\rangle \vee \varphi & \leftrightarrow\langle\langle\mathrm{Agt}\rangle\rangle \square \neg \varphi .
\end{aligned}
$$

We observe that all the properties presented in this subsection are schemes, rather than single formulae, and allow for uniform substitution. More precisely, $\varphi$ can be replaced by any state formula of ATL, and the resulting formula is a validity of ATL. Moreover, $\varphi$ in the duality axioms can be replaced by any state or path formula of $\mathbf{A T L}^{*}$, and the resulting formula is a validity of $\mathbf{A T L}$.

\section{Semantic variants: uncertainty and recall}

As we already pointed out in Sect. 2.4, one can distinguish between two types of strategies: an agent may base its decision on the current state or on the whole history of states that have occurred. Also, the agent may have complete or incomplete knowledge about the current global state of the system throughout the game. A number of semantic variations have been proposed for ATL, e.g. [3,4,35,39,43,45,57]. In this paper, we study the "canonical" variants as proposed in [57]. There, a natural taxonomy of four strategy types was introduced and labeled as follows: $R$ (resp. $r$ ) stands for perfect (resp. imperfect) recall, and $I$ (resp. $i$ ) refers to perfect (resp. imperfect) information. The semantics of ATL, $\mathbf{A T L}{ }^{+}$and $\mathbf{A T L}{ }^{*}$ can be parameterized with the strategy type-yielding four different semantic variants of the logic, labeled accordingly $\left(\mathbf{A T L} \mathbf{L}_{I R}, \mathbf{A T L} \mathbf{L}_{I r}, \mathbf{A T L}_{i R}, \mathbf{A T L} \mathbf{L}_{i r}\right.$, etc.).

In this paper, we extend the taxonomy with a distinction between objective and subjective abilities under imperfect information, denoted by $i_{o}$ and $i_{s}$, respectively; the distinction can be traced back to $[35,39,43,45]$. Intuitively, subjective ability to bring about $\gamma$ means that the agents are able to both identify and execute the right strategy, i.e., they not only can play to achieve $\gamma$; they also know how to do it. Objective ability is a weaker property: the agents could execute the right strategy, but they do not necessarily know which one works out, and they might be even unaware that such a strategy exists. Examples of agents who have objective but not subjective ability to achieve their goals include: garbage collecting robots that execute a strategy (program) provided by the producer, a Master's student executing a strategy hinted by his/her supervisor, etc.

The distinction between perfect and imperfect recall ( $R$ vs. $r$ ) is reflected in the type of function used to represent strategies $\left(S t^{+} \rightarrow A c t\right.$ vs. $\left.S t \rightarrow A c t\right)$. The distinction between perfect and imperfect information ( $I$ vs. $i$ ) yields constraints on the set of functions that represent "feasible" strategies. The additional refinement of the imperfect information case $\left(i_{o}\right.$ vs. $\left.i_{s}\right)$ determines which outcome paths will be taken into account when evaluating the success of a strategy.

\subsection{Imperfect information models and strategies}

Models, imperfect information concurrent game structures (iCGS) [57,61], are concurrent game structures augmented with a family of indistinguishability relations $\sim_{a} \subseteq S t \times S t$, one per agent $a \in \mathbb{A g t}$. The relations describe agents' uncertainty: $q \sim_{a} q^{\prime}$ means that agent $a$ cannot distinguish between states $q$ and $q^{\prime}$ of the system. Each $\sim_{a}$ is assumed to be an equivalence relation. It is also required that agents have the same choices in indistinguishable 
states. Note that CGS's can be seen as the subclass of iCGS's where all $\sim_{a}$ are the minimal reflexive relations. Formally, we have:

Definition 7 (iCGS) An imperfect information concurrent game structure (iCGS) is given by

$$
\mathfrak{M}=\left\langle\mathbb{A g t}, \text { St }, \Pi, \pi, A c t, d, o,\left\{\sim_{a} \mid a \in \mathbb{A g t}\right\}\right\rangle
$$

where $\langle\mathbb{A g t}, S t, \Pi, \pi, A c t, d, o\rangle$ is a CGS, each $\sim_{a} \subseteq S t \times S t$ is an equivalence relation, and if $q \sim_{a} q^{\prime}$ then $d(a, q)=d\left(a, q^{\prime}\right)$.

Definition 8 (Histories, $h \approx h^{\prime}$, last $\left.(h), \circ,|h|\right)$ A history is a finite sequence of states that can be effected by subsequent transitions. Two histories $h=q_{0} q_{1} \ldots q_{n}$ and $h^{\prime}=q_{0}^{\prime} q_{1}^{\prime} \ldots q_{n^{\prime}}^{\prime}$ are indistinguishable for agent $a\left(h \approx_{a} h^{\prime}\right)$ iff $n=n^{\prime}$ and $q_{i} \sim_{a} q_{i}^{\prime}$ for $i=0, \ldots, n$. Concatenation of $h$ and $h^{\prime}$ is denoted by $h \circ h^{\prime}$ or simply $h h^{\prime}$. We also use last $(h)$ to refer to the last state on history $h$, and $|h|$ to denote the length of $h$ (i.e., the number of states in $h$ ). As for paths, we use $h[i, j], i \leq j, i<|h|$, to refer to the subhistory $h[i] \ldots h[\min (j,|h|-1)]$. We do also allow $j=\infty$. We define $\Lambda_{\mathfrak{M}}^{\text {fin }}(q)$ as the set of all histories in $\mathfrak{M}$ starting from $q$, i.e., all the finite prefixes of paths in $\Lambda_{\mathfrak{M}}(q)$. Moreover, $\Lambda_{\mathfrak{M}}^{\text {fin }}:=\bigcup_{q \in S t} \Lambda_{\mathfrak{M}}^{\text {fin }}(q)$ denotes the set of all histories in $\mathfrak{M}$.

Additionally, for any equivalence relation $\mathcal{R}$ over a set $X$ we use $[x]_{\mathcal{R}}$ to denote the equivalence class of $x$. Moreover, we use the abbreviations $\sim_{A}:=\bigcup_{a \in A} \sim_{a}$ and $\approx_{A}:=$ $\bigcup_{a \in A} \approx_{a}$. We also write $\sim_{A}^{\mathfrak{M}}$ and $\approx_{A}^{\mathfrak{M}}$ if the model is not clear from the context. Note that relations $\sim_{A}$ and $\approx_{A}$ implement the "everybody knows" type of collective knowledge (i.e., $q$ and $q^{\prime}$ are indistinguishable for group $A$ iff there is at least one agent in $A$ for whom $q$ and $q^{\prime}$ look the same).

Definition 9 (ir-, iR-strategies) An imperfect information memoryless strategy (ir-strategy in short) is an $I r$-strategy satisfying the following additional constraint: if $q \sim_{a} q^{\prime}$ then $s_{a}(q)=s_{a}\left(q^{\prime}\right)$.

An imperfect information perfect recall strategy ( $i R$-strategy in short) of agent $a$ is an $I R$-strategy such that, if $h \approx_{a} h^{\prime}$, then $s_{a}(h)=s_{a}\left(h^{\prime}\right)$.

That is, strategy $s_{a}$ is a conditional plan that specifies $a$ 's action in each state of the system (for memoryless agents) or for every possible history of the system evolution (for agents with perfect recall). Moreover, imperfect information strategies specify the same choices for indistinguishable states (resp. histories).

Example 4 (Robots and Carriage, ctd.) We refine the scenario from Example 2 by restricting perception of the robots. Namely, we assume that robot 1 is only able to observe the color of the surface on which it is standing, and robot 2 perceives only the texture (cf. Fig. 2a). As a consequence, the first robot can distinguish between position 0 and position 1 , but positions 0 and 2 look the same to it. Likewise, the second robot can distinguish between positions 0 and 2 , but not 0 and 1 (cf. Fig. 2b).

Note that the strategy from Example 3 cannot be used anymore because it is not uniform (indeed, the strategy tells robot 1 to wait in $q_{0}$ and push in $q_{2}$ but both states look the same to the robot). 
(A)

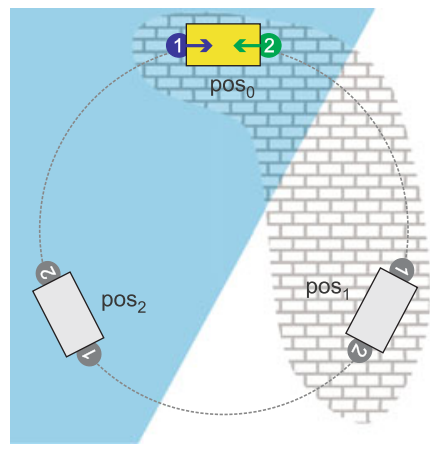

(B)

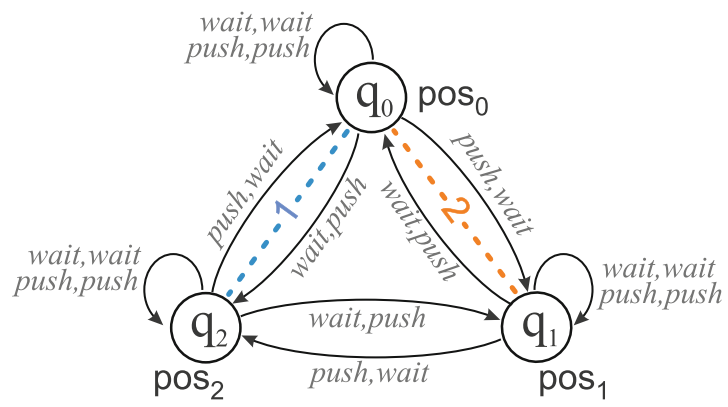

Fig. 2 Two robots and a carriage: a schematic view; b an imperfect information concurrent game structure $M_{0}^{\prime}$ that models the scenario. Dashed lines represent indistinguishability relations between states

\subsection{Subjective epistemic outcome}

Assumptions about agents' (un)certainty (i.e. the distinction between $I$ and $i$ ) and recall (i.e. the distinction between $R$ and $r$ ) are encoded in the mathematical structures that are used to represent strategies. However, if agent $a$ is to make sure that a strategy $s_{a}$ enforces property $\gamma$, it is not sufficient to consider only the paths from out $\left(q, s_{a}\right)$ because $a$ does not necessarily know that $q$ is the current state. To know that $s_{a}$ guarantees $\gamma$, agent $a$ should also check the outcome paths starting from states indistinguishable from $q$. From a conceptual point of view it makes sense to define two types of ability under imperfect information. Objective ability $\left(i_{o}\right)$ means that $a$ has an executable winning strategy, but the agent may be unaware of that, or be unable to identify the strategy on her own. Subjective ability $\left(i_{s}\right)$ requires that $a$ has a winning strategy and that $a$ can identify such a strategy, i.e., the agents knows how to play and not only that a good way of playing exists.

On the semantic side, this is reflected by the set of paths that are taken into account. Objective ability refers to the outcome paths that can objectively happen, while subjective ability builds on the outcome paths that are subjectively possible according to $a$ 's available information. ${ }^{6}$

Definition 10 (Subjective epistemic outcome, $x$-outcome) Let $\mathfrak{M}$ be an iCGS, $q$ a state in it and $s_{A}$ a collective strategy for group $A \subseteq \mathbb{A g t}$. Let $x \in\left\{i_{s}, i_{o}, I\right\}$. The $x$-outcome out $\mathfrak{M}_{\mathfrak{M}}^{x}\left(q, s_{A}\right)$ is defined as follows:

$$
\text { out }_{\mathfrak{M}}^{x}\left(q, s_{A}\right)= \begin{cases}\bigcup_{q \sim_{A} q^{\prime}} \text { out }_{\mathfrak{M}}\left(q^{\prime}, s_{A}\right) & \text { if } x=i_{s} \\ \operatorname{out}_{\mathfrak{M}}\left(q, s_{A}\right) & \text { else. }\end{cases}
$$

Again, we omit $\mathfrak{M}$ if it is clear from context.

Example 5 (Robots and Carriage) In the scenario from Example 4, a possible uniform strategy of robot 1 is to push in $q_{0}$ and $q_{2}$, and wait in $q_{1}$. If the starting state is $q_{0}$ then the strategy objectively makes sure that the system will never move to $q_{2}$. However, robot 1 does not know that the strategy is successful in avoiding $q_{2}$ since he must take into account also the outcome paths starting from $q_{2}$ which trivially violate the path property $\square \neg$ pos $_{2}$. Thus, 1 has the objective, but not the subjective, ability to enforce $\square \neg$ pos $_{2}$ in state $q_{0}$.

6 The issue is closely related to epistemic feasibility of plans, cf. e.g. [22,49]. 
In order to ensure a uniform notation, we introduce $x y$-strategies for $x \in\left\{i_{s}, i_{o}, I\right\}$ and $y \in\{r, R\}$ as follows:

IR: $s_{a}: S t^{+} \rightarrow$ Act such that $s_{a}\left(q_{0} \ldots q_{n}\right) \in d\left(a, q_{n}\right)$ for all $q_{0}, \ldots, q_{n}$;

Ir: $s_{a}: S t \rightarrow$ Act such that $s_{a}(q) \in d(a, q)$ for all $q$;

$i_{o} r, i_{s} r:$ like $I r$, with the additional constraint that $q \sim_{a} q^{\prime}$ implies $s_{a}(q)=s_{a}\left(q^{\prime}\right)$;

$i_{o} R, i_{s} R:$ like $I R$, with the additional constraint that $h \approx_{a} h^{\prime}$ implies $s_{a}(h)=s_{a}\left(h^{\prime}\right)$.

As before, collective xy-strategies $s_{A}$ are tuples of individual xy-strategies $s_{a}$, one per $a \in A$. We emphasize that $i_{s} y$-and $i_{o} y$-strategies are defined in the very same way, only the notion of outcome is different. Note also that the constraints in collective strategies refer to individual choices and individual relations $\sim_{a}\left(\right.$ resp. $\approx_{a}$ ), and not to collective choices and the derived relations $\sim_{A}\left(\right.$ resp. $\left.\approx_{A}\right)$.

\subsection{Unified setting: $x y$-semantics of ATL}

Finally, we put the pieces together and define the semantics of $\mathbf{A} \mathbf{T L} \mathbf{L}_{x y}, \mathbf{A} \mathbf{T} \mathbf{L}_{x y}^{+}$, and $\mathbf{A} \mathbf{T L} \mathbf{L}_{x y}^{*}$ for $x \in\left\{i_{s}, i_{o}, I\right\}$ and $y \in\{r, R\}$ by changing the clause for $\langle\langle A\rangle\rangle \gamma$ from Sect. 2.4 in the following way:

$\mathfrak{M}, q \models_{x y}\langle\langle A\rangle\rangle \quad$ iff there is an xy-strategy $s_{A}$ for agents $A$ such that for each path $\lambda \in \operatorname{out}^{x}\left(q, s_{A}\right)$, we have $\mathfrak{M}, \lambda \models \models_{x y} \gamma$.

Note, again, that the $I$ and $i_{o}$ semantics look only at outcome paths starting from the current global state of the system. In contrast, the $i_{S}$ semantics of $\langle\langle A\rangle\rangle \gamma$ refers to all outcome paths starting from states that look the same as the current state for coalition $A$. Hence, it formalizes the notion of $A$ knowing how to play in the sense that $A$ can identify a single strategy that succeeds from all the states they consider possible. We follow [57] by taking the "everybody knows" interpretation of collective uncertainty. More general settings were proposed in $[39,43]$. We believe that the results in this paper carry over to the other cases of "knowing how to play", too.

Example 6 (Robots and Carriage, ctd.) Consider the modified robots scenario from Example 4 (Fig. 2). With observational capabilities of the robots restricted in this way, no agent knows how to make the carriage reach or avoid any selected state singlehandedly from $q_{0}$, i.e., $M_{0}^{\prime}, q_{0} \models_{i_{s y}} \neg\langle\langle i\rangle\rangle \diamond$ pos $_{\mathrm{j}}$ and $M_{0}^{\prime}, q_{0} \models_{i_{s y}} \neg\langle\langle i\rangle\rangle \square \neg \operatorname{pos}_{\mathrm{j}}$ for all $y \in\{\mathrm{r}, \mathrm{R}\}, i \in$ $\{1,2\}, j \in\{1,2,3\}$. Note in particular that the strategy from Example 3 cannot be used here because it is not uniform, and the strategy from Example 5 does not succeed because of outcome paths from indistinguishable states. Still, the latter strategy can be used to demonstrate that robot 1 has the objective ability to avoid $q_{2}$ (though not $q_{1}$ anymore): $M_{0}^{\prime}, q_{0} \models_{i_{o y}}\langle\langle 1\rangle\rangle \square \neg \operatorname{pos}_{2} \wedge \neg\langle\langle i\rangle\rangle \square \neg$ pos $_{1}$.

The robots can keep the carriage away from pos $_{1}$ together, but only in the objective sense: $M_{0}^{\prime}, q_{0} \models_{i_{o y}}\langle\langle 1,2\rangle\rangle \square \neg$ pos $_{1}$. However, they cannot identify a strategy which guarantees that: $M_{0}^{\prime}, q_{0} \models_{i_{s y}} \neg\langle\langle 1,2\rangle\rangle \square \neg$ pos $_{1}$ (when in $q_{0}$, robot 2 considers it possible that the system is already in the "bad" state $q_{1}$ ). So, do the robots know how to play to achieve anything? Yes, for example they know how to make the carriage reach a given state eventually: $M_{0}^{\prime}, q_{0} \models_{i_{s y}}$ $\langle\langle 1,2\rangle\rangle \diamond$ pos $_{1}$ etc.-it suffices that one of the robots pushes all the time and the other waits all the time.

For the above properties the type of robots' recall does not matter (they hold in both memoryless and perfect recall strategies). $\langle\langle 1,2\rangle\rangle \triangleright \square$ pos $_{1}$ is an example formula that distinguishes between the two sets of strategies. Note that $M_{0}^{\prime}, q_{0} \models_{i_{o} r} \neg\langle\langle 1,2\rangle\rangle \diamond \square$ pos $_{1}$ : the robots have no memoryless strategy to bring the carriage to pos $_{1}$ and keep it there forever, even in the 
objective sense. Still, they have a successful perfect recall strategy for that, and are able to identify it: $M_{0}^{\prime}, q_{0} \models_{i_{S} R}\langle\langle 1,2\rangle\rangle \diamond \square$ pos $_{1}$. The right strategy is that one robot pushes and the other waits for the first 3 steps. After that, they know their current position exactly, and can go straight to position 1 and stay there.

\subsection{Folk result: memory does not matter for perfect information}

We observe that the basic semantics of $\mathbf{A T L}^{*}$ from [9] corresponds exactly to $\mathbf{A T L} \mathbf{L L}_{I R}^{*}$. A folk result states that, in the restricted language of ATL both semantics for perfect information coincide. That is, exactly the same formulae characterize models and their states in $\mathbf{A T L} \mathbf{L}_{I R}$ and $\mathbf{A T L}_{I r}$.

Proposition 1 For every $\boldsymbol{i C G S} \mathfrak{M}$, state $q$, and $\boldsymbol{A T L}$ formula $\varphi$, we have that $\mathfrak{M}, q \models_{I R} \varphi$ iff $\mathfrak{M}, q \models{ }_{I r} \varphi$.

Proof For finite models, equivalence of the semantics has been observed in [57], and follows from correctness of the model checking algorithm presented in [9]. It is not entirely obvious, however, that the result should extend to the infinite case. We present our own proof sketch below.

First, we observe that $\mathbf{A T L}_{I R}$ and $\mathbf{A} \mathbf{T L}_{I r}$ have the same validities. This follows from the results in [3] showing that: (1) perfect recall strategies in a CGS correspond to memoryless strategies in its tree unfolding, (2) every pointed CGS is strategically bisimilar to its tree unfolding, and (3) the same formulae of $\mathbf{A T L}_{I r}$ hold in strategically bisimilar models (cf. also a more detailed exposition in Sect. 4.1).

Now we can prove the equivalence of $\mathfrak{M}, q \models_{I R} \varphi$ and $\mathfrak{M}, q \models_{I r} \varphi$ by induction over the structure of $\varphi$. Cases $\varphi \equiv p, \neg \psi, \psi_{1} \wedge \psi_{2},\langle\langle A\rangle \bigcirc \psi$ are straightforward. For $\varphi \equiv\langle\langle A\rangle\rangle \square \psi$, we take the axiom schemes $\left(\mathbf{F P}_{\square}\right)$ and $\left(\mathbf{G F P}_{\square}\right)$ from [31]. It was proved in [31] that all their instances are validities of $\mathbf{A T L} \mathbf{L}_{I R}{ }^{7}$ By the previous observation, all the instances of schemes $(\mathbf{F P} \square)$ and $(\mathbf{G F P} \square)$ are validities of $\mathbf{A T L}_{I r}$ too. But that means that $\langle\langle A\rangle\rangle \square$ is the greatest fixpoint of the same monotone transformer of state sets in both semantics $\models_{I R}$ and $\models_{I r}$. Thus, the set of states satisfying $\langle\langle A\rangle\rangle$ in $\mathfrak{M}$ is the same in both semantics.

The proof for $\left\langle\langle A\rangle \psi_{1} \mathcal{U} \psi_{2}\right.$ is analogous, by showing that its extension in $\models_{I R}$ and $\models_{I r}$ is the least fixpoint of the same monotone transformer of state subsets from $\mathfrak{M}$.

Note that the $I R$ and $I r$ semantics coincide only for the restricted syntactic variant ATL. For $\mathbf{A T L}{ }^{*}$, and even $\mathbf{A T L}^{+}$, there are formulae that distinguish the two semantics, as we demonstrate in Sect. 5.1.

\section{Perfect recall and tree-like unfoldings}

Now we can turn to the original contribution of this paper. We begin by preparing the formal ground for our comparison of ATL validities under different semantics. In this section, we define several tree-like unfoldings of models, and show that they preserve truth of ATL formulae provided appropriate assumptions about agents' uncertainty and notion of success. This is the most technical part of the paper, needed mostly to prove the inclusion results in Sect. 5.1. However, its importance goes beyond technicalities. The unfoldings uncover some

7 The proof in [31] was for the class of finitely branching CGS (with possibly infinite state spaces) but it extends to the case of infinite branching in a straightforward way. 
of the conceptual structure that underlies ATL. In particular, they expose a "forgetting" phenomenon in the semantics of ATL: even agents with perfect recall are assumed to forget their past observations when proceeding to a subtask specified by a nested subformula (like in $\langle\langle a\rangle\rangle \diamond\langle\langle a\rangle\rangle \square \mathrm{p}$ ). In a way, one can talk about two variants of perfect recall: the "almost perfect recall" where agents use perfect recall strategies but abandon their previous observations when trying to enforce a nested strategic formula, and "truly perfect recall" where their hitherto observations carry over to the nested strategic task. On the other hand, our invariance theorems show that alternating-time temporal logic (even in its broadest syntactic variant $\mathbf{A} \mathbf{T L}{ }^{*}$ ) is too poor to distinguish between the two kinds of recall.

We believe that this section is of interest to readers who are intrigued by intricacies of game logics or search for tools that can be used to prove similar invariance results. On the other hand, readers interested only in the main conceptual contribution of this paper (i.e., the comparison of validities for variants of ATL) are advised to skip this part and proceed to Sect. 5 .

Plan of Sect. 4 A tree-like unfolding of an iCGS is an (infinite) model in which nodes correspond to finite sequences of states (i.e., histories) in the original iCGS. It is easy to see that the underlying transition structure of such an unfolding is a tree or a forest. The advantage of these structures is that perfect recall strategies and memoryless strategies coincide in treelike unfoldings. Moreover, each perfect recall strategy in the original model corresponds to a memoryless strategy in the unfolding yielding an equivalent outcome, and vice versa. Both properties are rather standard in the perfect information setting. For imperfect information, however, the constructions are more involved due to the specialities of the $i R$-semantics; more precisely, the knowledge of agents is "reset" whenever a nested strategic modality is evaluated.

For each of the three semantic settings of:

- perfect information,

- imperfect information with the objective semantics,

- imperfect information with the subjective semantics,

we proceed as follows:

1. We characterise appropriate tree-like structures and show that memoryless and perfect recall strategies coincide on them.

2. We define appropriate unfoldings and show that they result in tree-like structures.

3. We show that the unfoldings are truth-preserving (i.e. a formula which is true in the original model is also true in the tree-like unfolding and vice versa).

\subsection{Perfect information}

We begin with tree unfoldings of perfect information CGS's. We draw inspiration from the proof of [3, Theorem 8.3].

Definition 11 (Tree-like $\boldsymbol{C G S}, \rho_{\mathfrak{M}}\left(q_{1}, q_{2}\right)$ ) Let $\mathfrak{M}$ be a CGS. $\mathfrak{M}$ is called tree-like iff there is a state $q_{0}$ (the root) such that for every $q$ there is a unique history leading from $q_{0}$ to $q$.

Let $q_{1}$ and $q_{2}$ be states in $\mathfrak{M}$. If $q_{2}$ is reachable from $q_{1}$ then we use $\rho_{\mathfrak{M}}\left(q_{1}, q_{2}\right)$ to refer to the unique history from state $q_{1}$ to $q_{2}$; otherwise, if $q_{2}$ is not reachable from $q_{1}$ we set $\rho_{\mathfrak{M}}\left(q_{1}, q_{2}\right)=\epsilon$. Moreover, we use $\rho_{\mathfrak{M}}(q)$ as a shortcut for $\rho_{\mathfrak{M}}\left(q_{0}, q\right)$ (we will omit the subscript if clear from context). We note that $\rho_{\mathfrak{M}}\left(q_{0}\right)=q_{0}$. 
Every state $q$ in a tree-like CGS uniquely determines the path that leads from the root to $q$. Hence, perfect recall is already included in the states of the model. This is formally shown in the following proposition.

Proposition 2 (Recall invariance for tree-like CGS) For every tree-like CGS $\mathfrak{M}$, state $q$ in $\mathfrak{M}$, and $\boldsymbol{A T L}^{*}$-formula $\varphi$, we have: $\mathfrak{M}, q \models_{I r} \varphi$ iff $\mathfrak{M}, q \models_{I R} \varphi$.

Proof The proof is done by induction over the structure of $\varphi$.

Base cases:

Propositional case: Straightforward.

Case: $\varphi \equiv\langle\langle A\rangle\rangle \gamma$ where $\gamma$ contains no nested strategic modalities. The left-to-right direction is obvious. Now suppose that $\mathfrak{M}, q \models_{I R}\langle\langle A\rangle\rangle \gamma$ and let $s_{A}$ be a collective $I R$ strategy for $A$ such that for all $\lambda \in \operatorname{out}\left(q, s_{A}\right)$ it holds that $\mathfrak{M}, \lambda \models_{I R} \gamma$. We define $t_{A}\left(q^{\prime}\right)=s_{A}\left(\rho\left(q, q^{\prime}\right)\right)$ for each state $q^{\prime}$ in $\mathfrak{M}$ reachable from $q$. Then, $t_{A}$ is a well-defined $\operatorname{Ir}$-strategy with $\operatorname{out}\left(q, t_{A}\right)=\operatorname{out}\left(q, s_{A}\right)$. Hence, we have $\mathfrak{M}, q \models_{I r}\langle\langle A\rangle\rangle$.

Induction step:

Case: $\varphi \equiv \psi_{1} \wedge \psi_{2}$. Straightforward.

Case: $\varphi \equiv \neg \psi . \mathfrak{M}, q \models_{I r} \neg \psi$ iff not $\mathfrak{M}, q \models_{I r} \psi$ iff (by induction hypothesis) not $\mathfrak{M}, q \models_{I R} \psi$ iff $\mathfrak{M}, q \models_{I R} \neg \psi$.

Case: $\varphi \equiv\langle\langle A\rangle\rangle \gamma$. We observe that each state $q^{\prime}$ at which a state subformula $\psi$ of $\gamma$ is evaluated forms the root of a tree-like CGS. Then, by induction, $\psi$ has the same truth value in $q^{\prime}$ according to the $I R$ - and $I r$-semantics and can be replaced by a new atomic proposition with the appropriate valuation. This yields formula $\varphi^{\prime} \equiv\langle\langle A\rangle\rangle \gamma^{\prime}$ with no nested strategic modalities, to which we apply the same argument as above.

A natural question is whether every model has an equivalent tree-like CGS. By "equivalent" we mean that the sets of formulae which hold at corresponding states are always the same.

Definition 12 (Tree unfolding) Let $\mathfrak{M}=(\mathbb{A g t}, S t, \Pi, \pi, A c t, d, o)$ be a CGS and $q$ be a state in it. The (perfect information) tree unfolding of the pointed model (M, $q$ ) denoted $T(\mathfrak{M}, q)$ is defined as (Agt, $\left.S t^{\prime}, \Pi, \pi^{\prime}, A c t, d^{\prime}, o^{\prime}\right)$ where

- $S t^{\prime}:=\Lambda_{\mathfrak{M}}^{\text {fin }}(q)$,

$-d^{\prime}(a, h):=d(a, \operatorname{last}(h))$,

$-o^{\prime}(h, \boldsymbol{\alpha}):=h \circ o(\operatorname{last}(h), \boldsymbol{\alpha})$, and

$-\pi^{\prime}(h):=\pi($ last $(h))$.

The node $q$ in the unfolding is called the root of $T(\mathfrak{M}, q)$.

An example tree unfolding is shown in Fig. 3. It is important to note that histories in $\mathfrak{M}$ are states in $T(\mathfrak{M}, q)$ and that each tree unfolding is tree-like:

Proposition 3 The tree unfolding of a pointed CGS $(\mathfrak{M}, q)$ is tree-like.

We now show that satisfaction of ATL*-formulae is invariant under tree unfoldings and that memory is not needed in the tree unfolding. 


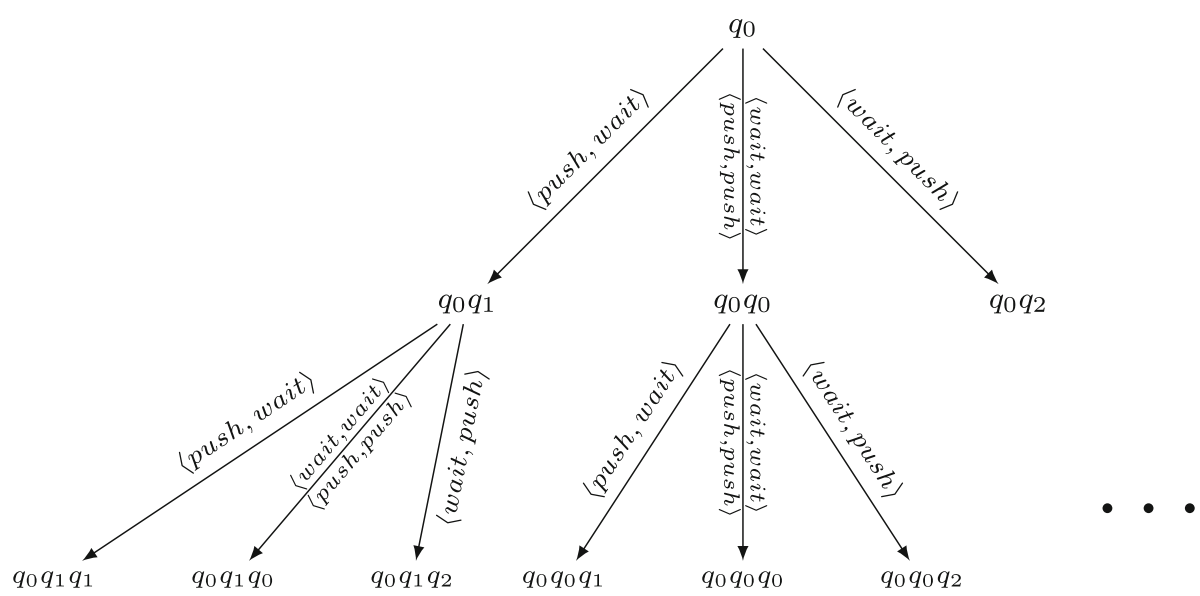

Fig. 3 Tree unfolding $T\left(M_{0}, q_{0}\right)$ for the robots and carriage CGS from Fig. 1

Theorem 1 For every CGS $\mathfrak{M}$, state $q$ in $\mathfrak{M}$, and $\boldsymbol{A T L}^{*}$-formula $\varphi$ we have: ${ }^{8}$

$$
\mathfrak{M}, q \models_{I R} \varphi \text { iff } T(\mathfrak{M}, q), q \models_{I R} \varphi \text { iff } T(\mathfrak{M}, q), q \models_{I r} \varphi .
$$

Proof The second equivalence follows from Propositions 2 and 3. To prove the first equivalence we show that, for all $h \in \Lambda_{\mathfrak{M}}^{f i n}(q)$, we have $\mathfrak{M}$, last $(h) \models_{I R} \varphi$ iff $T(\mathfrak{M}, q), h \models_{I R} \varphi$ (by induction over the structure of $\varphi$ ). Note that for each state $q^{\prime}$ reachable from $q$ in $\mathfrak{M}$ there is a history $h$ such that last $(h)=q^{\prime}$.

Base cases:

Propositional case: Straightforward.

Case: $\varphi \equiv\langle\langle A\rangle\rangle \gamma$ where $\gamma$ does not contain any strategic modalities.

“ $\Rightarrow$ ": Suppose that $\mathfrak{M}$, last $(h) \models_{I R} \varphi$. Then, there is an IR-strategy $s_{A}$ such that for all $\lambda \in$ out $\left(\right.$ last $\left.(h), s_{A}\right)$ we have that $\mathfrak{M}, \lambda \models \gamma$. Now let $t_{A}$ be an Ir-strategy defined as follows: $t_{A}\left(h h^{\prime}\right)=s_{A}\left(\right.$ last $\left.(h) h^{\prime}\right)$, and arbitrary otherwise. By definition of the tree unfolding and the construction of $t_{A}$ we have that

$$
\begin{aligned}
& \text { last }(h) q_{1} q_{2} \cdots \in \text { out }_{\mathfrak{M}}\left(\text { last }(h), s_{A}\right) \text { iff } \\
& (h)\left(h q_{1}\right)\left(h q_{1} q_{2}\right) \cdots \in \operatorname{out}_{T(\mathfrak{M}, q)}\left(h, t_{A}\right) .
\end{aligned}
$$

Since the valuation of propositions only depends on the final state of a history and since $\mathrm{Ir}$ strategies can be seen as special cases of $I R$-strategies, we have also that $T(\mathfrak{M}, q), h \models_{I R}$ $\varphi$.

"Æ”: Suppose that $T(\mathfrak{M}, q), h \models_{I R} \varphi$. Then, by Propositions 2 and 3 , there is an Irstrategy $s_{A}$ such that for all $\lambda \in \operatorname{out}\left(h, s_{A}\right)$ we have that $T(\mathfrak{M}, q), \lambda \models \gamma$. We define the following $I R$-strategy $t_{A}$ :

$$
t_{A}\left(h^{\prime}\right)= \begin{cases}s_{A}\left(h\left(h^{\prime}[1, \infty]\right)\right) & \text { if } h^{\prime}[0]=\text { last }(h) \\ \alpha & \text { else, for some arbitrary } \alpha \in d_{A}\left(\text { last }\left(h^{\prime}\right)\right)\end{cases}
$$

8 The equivalence of $\mathfrak{M}, q \models_{I R} \varphi$ and $T(\mathfrak{M}, q), q \models_{I R} \varphi$ follows also from the results on alternating bisimulation, cf. [10] for the bisimulation in finite models, and [3] for the general case. We present the construction nevertheless, as it will be adapted in the following sections to the case of imperfect information. 
The first case of the definition $t_{A}$ applies if $h^{\prime}[1, \infty]$, i.e. $h^{\prime}$ without the initial state, is a possible extension of history $h$. The history $h\left(h^{\prime}[1, \infty]\right)$ is the extension of $h$ with $h^{\prime}$ where the last state of $h$ or the initial state of $h^{\prime}$ has to be removed as it occurs twice. Again, we have

$$
\begin{array}{r}
\text { last }(h) q_{1} q_{2} \cdots \in \text { out }_{\mathfrak{M}}\left(\operatorname{last}(h), t_{A}\right) \text { iff } \\
(h)\left(h q_{1}\right)\left(h q_{1} q_{2}\right) \cdots \in \operatorname{out}_{T(\mathfrak{M}, q)}\left(h, s_{A}\right)
\end{array}
$$

and thus $\mathfrak{M}$, last $(h) \models_{I R} \varphi$.

Induction step:

Case: $\varphi \equiv \psi_{1} \wedge \psi_{2}$. Straightforward.

Case: $\varphi \equiv \neg \psi$. Straightforward.

Case: $\varphi \equiv\langle\langle A\rangle\rangle \gamma$. We observe that for each state $q^{\prime}$ in $\mathfrak{M}$ reachable from $q$ at which a state-subformula $\varphi^{\prime}$ of $\gamma$ is evaluated there is a history $h$ such that $T(\mathfrak{M}, q)$ contains a state $h q^{\prime}$ at which the very subformula holds (by induction hypothesis). Then we apply the same reasoning as for the case with no nested strategic modalities.

\subsection{Imperfect information: objective ability}

Unlike in the perfect information case, tree unfoldings for imperfect information must also take into account the indistinguishability relations. We construct our argument for the $i_{o}$ case similarly to Sect. 4.1. The notion of tree-like imperfect information CGS has to include suitable constraints on the epistemic relations-otherwise we would not get truth invariance with respect to recall. To handle the issue, we introduce objective epistemic tree unfoldings under perfect recall, or $i_{o} R$-tree unfoldings in short.

Definition 13 (Tree structure of $\boldsymbol{i C G S}$ ) Let $\mathfrak{M}$ be an iCGS. $\mathfrak{M}$ has tree structure iff the underlying CGS of $\mathfrak{M}$ (i.e., $\mathfrak{M}$ without epistemic relations) is tree-like. As in Definition 11 we use $\rho_{\mathfrak{M}}\left(q_{1}, q_{2}\right)$ to refer to the unique history between $q_{1}$ and $q_{2}$ in $\mathfrak{M}$ if it exists and set $\rho_{\mathfrak{M}}(q)=\rho_{\mathfrak{M}}\left(q_{0}, q\right)$ where $q_{0}$ is the root in $\mathfrak{M}$. Again, we omit $\mathfrak{M}$ from $\rho_{\mathfrak{M}}(\cdot)$ if clear from context.

Definition $14\left(i_{o} R\right.$-tree-like) Let $\mathfrak{M}$ be an $\mathbf{C G S}$ with tree structure. $\mathfrak{M}$ is called $i_{o} R$-tree-like iff for all $a \in \mathbb{A g t}$ and all $q_{1}, q_{2} \in S t$ we have $q_{1} \sim \underset{a}{\mathfrak{M}} q_{2}$ iff $\rho\left(q_{1}\right) \approx_{a}^{\mathfrak{M}} \rho\left(q_{2}\right)$. (We note that $\rho\left(q_{1}\right) \approx_{a}^{\mathfrak{M}} \rho\left(q_{2}\right)$ implies $q_{1} \underset{a}{\sim} \mathfrak{M} q_{2}$ by definition of $\approx_{a}^{\mathfrak{M}}$.)

In other words, in an $i_{o} R$-tree-like structure the information sets in a game can only be more precise when the game already follows some previous interaction. The next proposition is analogous to Proposition 2.

Proposition 4 (Recall invariance for $i_{o} R$-tree-like models) For every $i_{o} R$-tree-like $\mathbf{i C G S} \mathfrak{M}$, state $q$ in $\mathfrak{M}$, and $\boldsymbol{A T L}{ }^{*}$-formula $\varphi$, we have that $\mathfrak{M}, q \models_{i_{o} r} \varphi$ iff $\mathfrak{M}, q \models_{i_{o} R} \varphi$.

Proof Induction over the structure of $\varphi$.

Base cases:

Propositional case: Straightforward.

Case: $\varphi \equiv\langle\langle A\rangle\rangle \gamma$ where $\gamma$ contains no strategic modalities. The left-to-right direction is obvious. Now suppose that $\mathfrak{M}, q \models_{i_{o} R}\langle\langle A\rangle\rangle$ and let $s_{A}$ be a collective $i R$-strategy for $A$ such that for all $\lambda \in \operatorname{out}\left(q, s_{A}\right)$ it holds that $\mathfrak{M}, \lambda \models_{i_{o} R} \gamma$. We define $t_{a}\left(q^{\prime}\right)=s_{a}\left(\rho\left(q, q^{\prime}\right)\right)$ 


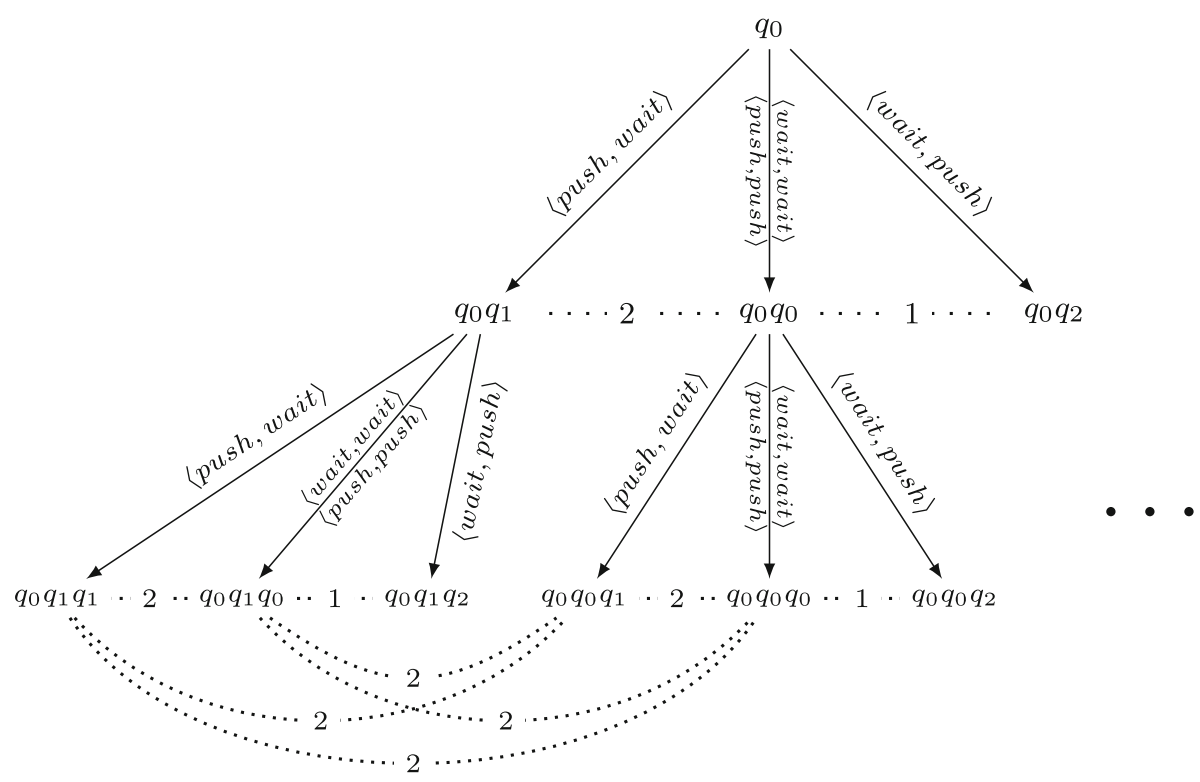

Fig. $4 i_{o} R$-tree unfolding $T_{o}\left(M_{0}^{\prime}, q_{0}\right)$ of the robots with limited information from Fig. 2 (we omitted reflexive epistemic links)

for each state $q^{\prime}$ in $\mathfrak{M}$ which is reachable from $q$. Then, we have out $\left(q, t_{A}\right)=\operatorname{out}\left(q, s_{A}\right)$. Moreover, we have for all states $q_{1}$ and $q_{2}$ and all agents $a \in \mathbb{A g t}$ that $q_{1} \sim_{a} q_{2}$ iff $\rho\left(q_{1}\right) \approx_{a} \rho\left(q_{2}\right)$ because: (a) the right-to-left direction is clear from the definition of $\approx$, and (b) the left-to-right direction follows because $\mathfrak{M}$ is $i_{o} R$-tree-like. Hence, $t_{A}$ is a well-defined $i_{o} r$-strategy with $\operatorname{out}\left(q, t_{A}\right)=\operatorname{out}\left(q, s_{A}\right)$ and thus: $\mathfrak{M}, q \models_{i_{o} r}\langle\langle A\rangle\rangle$.

Induction step:

Case: $\varphi \equiv \psi_{1} \wedge \psi_{2}$. Straightforward.

Case: $\varphi \equiv \neg \psi \cdot \mathfrak{M}, q \models_{i_{o} r} \neg \psi$ iff not $\mathfrak{M}, q \models_{i_{o} r} \psi$ iff (by induction hypothesis) not $\mathfrak{M}, q \models_{i_{o} R} \psi$ iff $\mathfrak{M}, q \models_{i_{o} R} \neg \psi$.

Case: $\varphi \equiv\langle\langle A\rangle\rangle$. We observe that each state $q^{\prime}$ at which a state subformula $\psi$ of $\gamma$ is evaluated forms the root of a $i_{o} R$-tree-like iCGS. Then, by induction, $\psi$ has the same truth value in $q^{\prime}$ according to the $i_{o} R$ - and $i_{o} r$-semantics and can be replaced by a new atomic proposition with the appropriate valuation. This yields formula $\varphi^{\prime} \equiv\left\langle\langle A\rangle \gamma^{\prime}\right.$ with no nested strategic modalities, to which we apply the same argument as above.

Now, the $i_{o} R$-tree unfolding is defined as standard tree unfolding for the perfect information case extended with indistinguishability relations between histories of the model (which are nodes of the unfolding).

Definition 15 ( $i_{o} R$-tree unfolding) Given an iCGS $\mathfrak{M}$ and a state $q$ in it, we define the $i_{o} R$-tree unfolding of $(\mathfrak{M}, q)$, denoted $T_{o}(\mathfrak{M}, q)$, as $T(\mathfrak{M}, q)$ from Definition 12 extended with epistemic relations $\sim_{a}^{T_{o}(\mathfrak{M}, q)}$ reflecting indistinguishability of histories in $\mathfrak{M}$; that is, $h \sim_{a}^{T_{o}(\mathfrak{M}, q)} h^{\prime}$ iff $h \approx_{a}^{\mathfrak{M}} h^{\prime}$ where $h$ and $h^{\prime}$ start in $q$.

As an example, the $i_{o} R$-tree unfolding of the robots and carriage iCGS is presented in Fig. 4. 


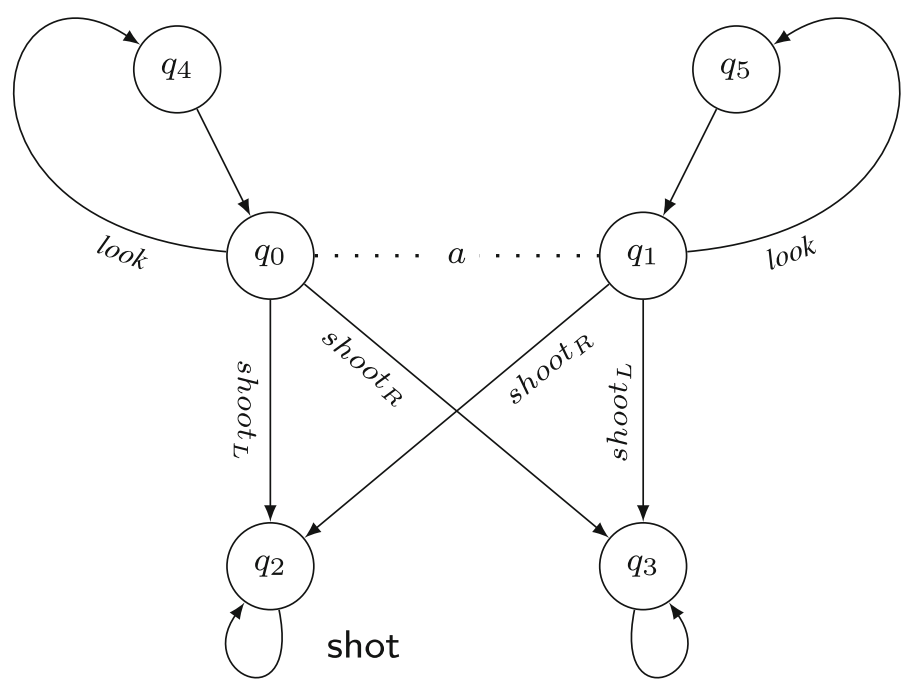

Fig. 5 "Poor duck model" $M_{1}$ with one player $(a)$ and transitions labeled with $a$ 's actions. The dotted line depicts $a$ 's indistinguishability relation. Automatic transitions (i.e., such that there is only one possible transition from the starting state) are left unlabeled

Proposition 5 Let $(\mathfrak{M}, q)$ be a pointed $\boldsymbol{i C G S}$. The $i_{o} R$-tree unfolding of $(\mathfrak{M}, q)$ is $i_{o} R$-treelike.

Proof Clearly, the unfolding has tree structure and is $i_{o} R$-tree-like by definition of the indistinguishability relations in the $i_{o} R$-tree-unfolding.

Analogously to Theorem 1 we have that $i_{o} R$-tree unfoldings are truth preserving and that memory does not matter in these unfolded models.

Theorem 2 For every iCGS $\mathfrak{M}$, state $q$ in $\mathfrak{M}$, and $\boldsymbol{A T L}^{*}$-formula $\varphi$ we have:

$$
\mathfrak{M}, q \models_{i_{o} R} \varphi \text { iff } T_{o}(\mathfrak{M}, q), q \models_{i_{o} R} \varphi \text { iff } T_{o}(\mathfrak{M}, q), q \models_{i_{o} r} \varphi .
$$

The proof is given in the Appendix.

\subsection{Imperfect information: subjective ability}

The case for the subjective semantics $\left(i_{s}\right)$ cannot be proven in the same way by using $i_{o} R$ tree unfoldings. Obviously, when constructing an unfolding of $(\mathfrak{M}, q)$ for the $i_{s} R$-semantics one has to take into account paths starting from states indistinguishable from $q$. A first naive approach could be to define the $i_{S} R$-unfolding as a structure consisting of $i_{o} R$-tree unfoldings, one for each epistemic alternative, and to connect the root nodes of all these unfoldings. Unfortunately, this simple idea is not sufficient as illustrated in Example 7.

Example 7 (First naive approach to $i_{S} R$-tree unfoldings) We consider the iCGS $M_{1}$ shown in Fig. 5. The story is as follows. A man wants to shoot down a yellow rubber duck in a shooting gallery. The man knows that the duck is in one of the two cells in front of him, but he does not know in which one. He can shoot to the left (action $s_{h o o t}$ ) or to the right $\left(\operatorname{shoot}_{R}\right)$. Alternatively, he can reach out and open one of the cells for a moment (action look), thus removing his uncertainty. 


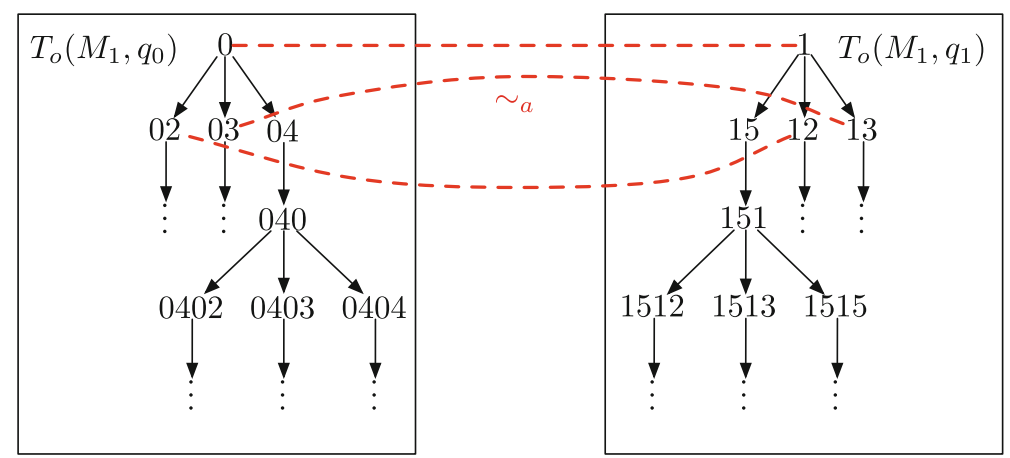

Fig. 6 Two $i_{o} R$-tree unfoldings connected by epistemic links. Each label $i_{1} i_{2} \ldots$ refers to the history $q_{i_{1}} q_{i_{2}} \ldots$

Let us take the $i_{o} R$-tree unfoldings $T_{o}\left(M_{1}, q_{0}\right)$ and $T_{o}\left(M_{1}, q_{1}\right)$, and interconnect their nodes by epistemic links whenever the corresponding histories are indistinguishable in the original model. The resulting model is shown in Fig. 6 (we will call the model $T_{1}$ ). Unfortunately, this construction is not truth-preserving. That is because if a state-subformula is evaluated in states 040 and 151 of $T_{1}$ the agent will know where the game is-which is not consistent with the $i_{s}$ semantics: only the last state of each history should be considered.

To be more precise, let us consider formula $\varphi=\langle\langle a\rangle\rangle \bigcirc\langle\langle a\rangle\rangle\langle\langle a\rangle\rangle \bigcirc$ shot. Clearly, we have $M_{1}, q_{0} \not \nvdash_{i_{s}} R \varphi$. On the other hand, we have $T_{1}, q_{0} \models_{i_{s} R} \varphi$.

In order to improve the naive approach one may simply add an epistemic link between states 040 and 151. Unfortunately, this does not work either. Such a link indicates that the states 040 and 151 are indistinguishable for $a$; on the other hand, player $a$ can distinguish the histories which lead to these states. This contradicts the conceptual idea in which states are associated with histories. Moreover, it is easy to construct a concrete counterexample.

To make the observation in Example 7 more formal, suppose $h q$ is some node in the $i_{o} R$ tree unfolding $T_{o}\left(\mathfrak{M}, q_{0}\right)$ and that in this node a formula $\langle\langle a\rangle\rangle \gamma$ is evaluated. Then, $\langle\langle a\rangle\rangle \gamma$ holds iff agent $a$ has a successful $i R$-strategy not only for all paths starting from $h q$, but also for paths starting from nodes $h^{\prime} q^{\prime}$ such that $q \sim \underset{a}{\mathfrak{M}} q^{\prime}$. In the $i_{o} R$-tree unfolding, however, these nodes are usually not linked via an epistemic transition. On the other hand, we cannot simply introduce the link $h q \sim_{a}^{T_{o}\left(\mathfrak{M}, q_{0}\right)} h^{\prime} q^{\prime}$ as we would loose soundness of the construction (in general, $h$ and $h^{\prime}$ do not need to be indistinguishable). This observation makes it necessary to introduce a more sophisticated construction for the subjective epistemic tree-like unfoldings under perfect recall, or $i_{S} R$-pando unfoldings in short. ${ }^{9}$

Firstly, we discuss when an iCGS should be considered $i_{S} R$-pando-like. The idea of a set of connected $i_{o} R$-tree-like models (like in Example 7) seems to come close. However, we should also account for the "forgetting" of the history of the play when a nested strategic operator is evaluated. This is because if a state subformula (like $\langle\langle a\rangle\rangle \gamma$ ) is evaluated in a history $h$, only the last state of $h$ is relevant. The rest of $h$ is "lost" as it does not influence the truth of $\langle\langle a\rangle\rangle \gamma$ in $h$. We deal with it by adding appropriate "hanging" trees with roots $q$ that are indistinguishable from last $(h)$ in the original models. The new trees are connected

\footnotetext{
9 We thank an anonymous reviewer of JAAMAS for the excellent terminological suggestion. An $i_{S} R$ unfolding is not a tree, as it usually consists of several transition trees. On the other hand, it is not a typical forest because the trees are not separate - they are intimately connected by epistemic relations. For the biological Pando, see for example http://en.wikipedia.org/wiki/Pando(tree).
} 
to histories in the "basic" tree by appropriate epistemic links. We must also make sure that there are no epistemic links between such trees apart from the ones just explained.

Definition $16\left(i_{S} R\right.$-pando-like, $\left.\rho_{\mathfrak{M}}\left(q, q^{\prime}\right)\right)$ An iCGS

$$
\mathfrak{M}=\left\langle\mathbb{A g t}, \text { St }, \Pi, \pi, \text { Act }, d, o,\left\{\sim_{a}\right\}_{a \in \mathbb{A g t}}\right\rangle
$$

is $i_{S} R$-pando-like iff it consists of submodels

$$
\mathfrak{M}_{i}=\left\langle\mathbb{A g t}, S t_{i}, \Pi, \pi_{i}, A c t, d_{i}, o_{i},\left\{\stackrel{\mathfrak{M}_{i}}{\}_{a \in \mathbb{A g t}}}\right\rangle\right.
$$

for $i \in I$ and some index set $I \subseteq \mathbb{N}$, where:

- each $\mathfrak{M}_{i}$ is an $i_{o} R$-tree-like iCGS,

- $S t=\biguplus_{i \in I} S t_{i}$ (i.e. the states of the $\mathfrak{M}_{i}$ 's form a partition of $\mathfrak{M}$ ),

$-\pi: S t \rightarrow 2^{\Pi}$ with $\pi(q)=\pi_{i}(q)$ for $q \in S t_{i}$,

- $d: S t \times \mathbb{A g t} \rightarrow 2^{A c t}$ with $d(q, a)=d_{i}(q, a)$ for $q \in S t_{i}$,

- o :St $\times A c t^{|\mathrm{Agt}|} \rightarrow S t$ with $o(q, \alpha)=o_{i}(q, \alpha)$ for $q \in S t_{i}$, and

- $\sim_{a} \subseteq S t \times S t$ with $\sim_{a}:=\left(\bigcup_{i \in I} \sim_{a}^{\mathfrak{M}_{i}}\right) \cup \hat{\sim}_{a}$ where each $\hat{\sim}_{a} \subseteq \bigcup_{i \in I} S t_{i} \times \bigcup_{i \in I} S t_{i}$,

and the following conditions are satisfied:

1. the relation $\sim_{a}$ is transitive for every $a \in \mathbb{A g t}$.

2. $\hat{\sim}_{a}$ is a symmetric relation for every $a \in \mathbb{A g t}$.

3. for all $i \in I$ we have $\hat{\sim}_{a} \cap\left(S t_{i} \times S t_{i}\right)=\emptyset$ (the relation does only exist between different $i_{o} R$-tree like models).

4. for $q_{1} \in S t_{i}$ and $q_{2} \in S t_{j}$ with $i, j \in I, i \neq j$, we have that if $q_{1} \hat{\sim}_{a} q_{2}$ then $\rho_{\mathfrak{M}_{i}}\left(q_{1}\right) \hat{\approx}_{a}^{\mathfrak{M}} \rho_{\mathfrak{M}_{j}}\left(q_{2}\right)$ or $\rho_{\mathfrak{M}_{i}}\left(q_{1}\right)=q_{1}$ or $\rho_{\mathfrak{M}_{j}}\left(q_{2}\right)=q_{2}$ where for two histories $h$ and $h^{\prime}$ we have $h \hat{\approx}_{a}^{\mathfrak{M}} h^{\prime}$ iff $|h|=\left|h^{\prime}\right|$ and $h[l] \hat{\sim}_{a} h^{\prime}[l]$ for all $l=0, \ldots,|h|-1$ (indistinguishable nodes in different $i_{o} R$-tree-like models must have indistinguishable histories or at least one of the nodes is a root node).

5. for $q_{1}, q_{2} \in S t_{i}$ and $\sim_{\mathbb{A g t}}=\bigcup_{a \in \mathbb{A g t}} \sim_{a}$, if $q_{1}\left(\sim_{\mathbb{A g t}}\right)^{*} q_{2}$ then $\left|\rho_{\mathfrak{M}_{i}}\left(q_{1}\right)\right|=\left|\rho_{\mathfrak{M}_{i}}\left(q_{2}\right)\right|$ (nodes in the same tree indistinguishable for a group must be on the same level). The idea behind this condition is illustrated in Fig. 7.

Moreover, we define $\rho_{\mathfrak{M}}\left(q_{1}, q_{2}\right)$ as $\rho_{\mathfrak{M}_{i}}\left(q_{1}, q_{2}\right)$ for $q_{1}, q_{2} \in S t_{i}$ and set $\rho_{\mathfrak{M}}\left(q_{1}, q_{2}\right)=\epsilon$ if $q_{1} \in S t_{i}$ and $q_{2} \in S t_{j}$ for $i \neq j$.

Remark 1 We would like to note that it is possible, due to condition 4 of Definition 16, to weaken condition 5 of Definition 16 to the following: Let $q_{1} \in S t_{i}, q_{2} \in S t_{j}, i, j \in I, i \neq j$, $q_{1}\left(\sim_{\mathbb{A g t}}\right)^{*} q_{2}$, and $\rho_{\mathfrak{M}_{j}}\left(q_{2}\right)=q_{2}$ where $\sim_{A}=\bigcup_{a \in A} \sim_{a}$ for $A \subseteq \mathbb{A g t}$. If there is an $q_{1}^{\prime} \in S t_{i}$, with $q_{2}\left(\sim_{\mathbb{A g t}}\right)^{*} q_{1}^{\prime}$; then, $\left|\rho_{\mathfrak{M}_{i}}\left(q_{1}\right)\right|=\left|\rho_{\mathfrak{M}_{i}}\left(q_{1}^{\prime}\right)\right|$.

Before we give an intuitive example we show that the concept of $i_{S} R$-pando-like iCGS is well-defined.

Proposition 6 Let $\mathfrak{M}$ be an $i_{s} R$-pando like $\boldsymbol{i C G S}$ as defined in Definition 16. Each relation $\sim_{a}$ is an equivalence relation for $a \in \mathbb{A g t}$.

Proof By definition, each $\sim_{a}$ is transitive. Symmetry follows from the symmetry of $\hat{\sim}_{a}$ and of $\sim_{a}^{\mathfrak{M}_{i}}$. Reflexivity of $\sim_{a}^{\mathfrak{M}_{i}}$ does also imply reflexivity of $\sim_{a}$. 
Fig. 7 Condition 5 of Definition 16

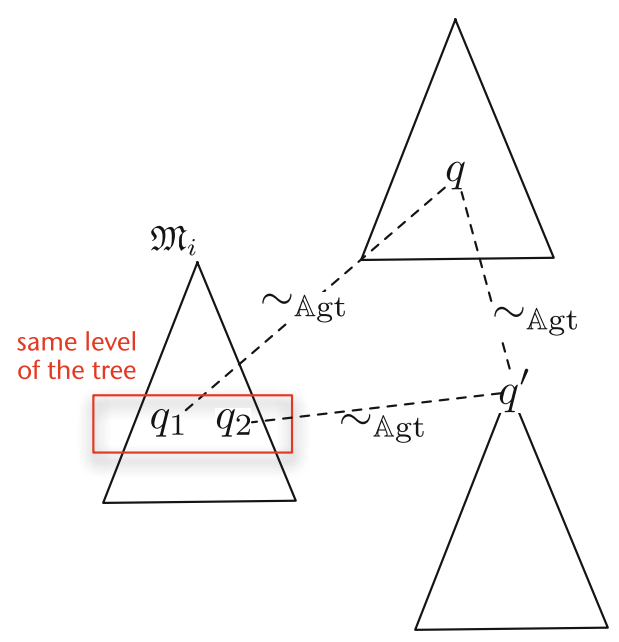

Example 8 Figure 8 depicts an $i_{S} R$-pando-like iCGS. In fact, the model shows a suitable unfolding of the pointed iCGS $\left(M_{1}, q_{0}\right)$ from Fig. 5 . We will formally introduce $i_{S} R$-pando unfoldings in Definition 17.

In the spirit of Propositions 2 and 4 we have that memory is not needed in $i_{s} R$-pandolike models. The proof for the left-to-right direction is obvious. The sophisticated step is to construct an $i_{S} r$-strategy from an $i_{S} R$-strategy. For the sake of readability we have moved the technical part in the appendix (Lemma 3).

Proposition 7 (Recall invariance for $i_{S} R$-pando-like models) For every $i_{S} R$-pando-like $i$ CGS $\mathfrak{M}$, state $q$ in $\mathfrak{M}$, and $\boldsymbol{A T L}{ }^{*}$-formula $\varphi$, we have that $\mathfrak{M}, q \models_{i_{s} r} \varphi$ iff $\mathfrak{M}, q \models_{i_{s} R} \varphi$.

Proof Firstly, we recall that all the "subpandos" which form an $i_{o} R$-tree-like iCGS are not interconnected by transitions and thus the path to each state is unique. The proof is done analogously to Proposition 4; we only consider the important base case where $\varphi \equiv\langle\langle A\rangle\rangle$ and $\gamma$ contains no strategic modalities. The left-to-right direction is obvious.

For the right-to-left direction suppose that $\mathfrak{M}, q \models_{i_{s} R}\langle\langle A\rangle\rangle$ and let $s_{A}$ be a collective $i R$-strategy for $A$ such that for all $\lambda \in o u t^{i_{s}}\left(q, s_{A}\right)$ it holds that $\mathfrak{M}, \lambda \models_{i_{s} R} \gamma$. Let $q^{\prime} \in S t_{j}$ be a state reachable from $\hat{q} \in S t_{j}$ with $\hat{q} \sim \underset{a}{\mathfrak{M}} q$ for some $a \in A$. Then, we define the memoryless strategy $t_{a}$ as follows: $t_{a}\left(q^{\prime}\right)=s_{a}\left(\rho_{\mathfrak{M}_{j}}\left(\hat{q}, q^{\prime}\right)\right)$. We proceed like this for all states $q^{\prime}, \hat{q}$ and define the strategies $t_{a}$ arbitrarily but in a uniform way for all other states in $\mathfrak{M}$. (Note, that these are all states which are not reachable from any epistemic alternative of $q$ for some agent in A.) Firstly, we observe that each $t_{a}$ is well-defined as each $\mathfrak{M}_{j}$ is $i_{o} R$-tree-like and thus the path $\rho_{\mathfrak{M}_{j}}\left(\hat{q}, q^{\prime}\right)$ to a state $q^{\prime}$ is unique.

In order to show that each $t_{a}$ is uniform and that $o u t^{i_{s}}\left(q, t_{A}\right)=o u t^{i_{s}}\left(q, s_{A}\right)$ we have to prove that for any two states $q_{1}$ reachable from $\hat{q}_{1}$, and $q_{2}$ reachable from $\hat{q}_{2}$ with $q_{1} \sim \underset{a}{\mathfrak{M}} q_{2}$ and $\hat{q}_{1}, \hat{q}_{2} \in\left\{q^{\prime} \in S t \mid q \sim_{A} q^{\prime}\right\}$ (i.e. $\hat{q}_{1} \sim_{b}^{\mathfrak{M}} q \sim_{c}^{\mathfrak{M}} \hat{q}_{2}$ for some $b, c \in A$ ) we also have $\rho\left(\hat{q}_{1}, q_{1}\right) \approx_{a}^{\mathfrak{M}} \rho\left(\hat{q}_{2}, q_{2}\right)$. This part is shown in Lemma 3 in the Appendix.

The basic idea of the subjective epistemic pando unfolding under perfect recall $\left(i_{s} R\right.$ pando unfolding in short) is to create copies of the tree starting in $q^{\prime}$, one for each epistemic alternative. Then, we can link $h q$ with these new root nodes $q^{\prime}$ of the "copies" of the trees 


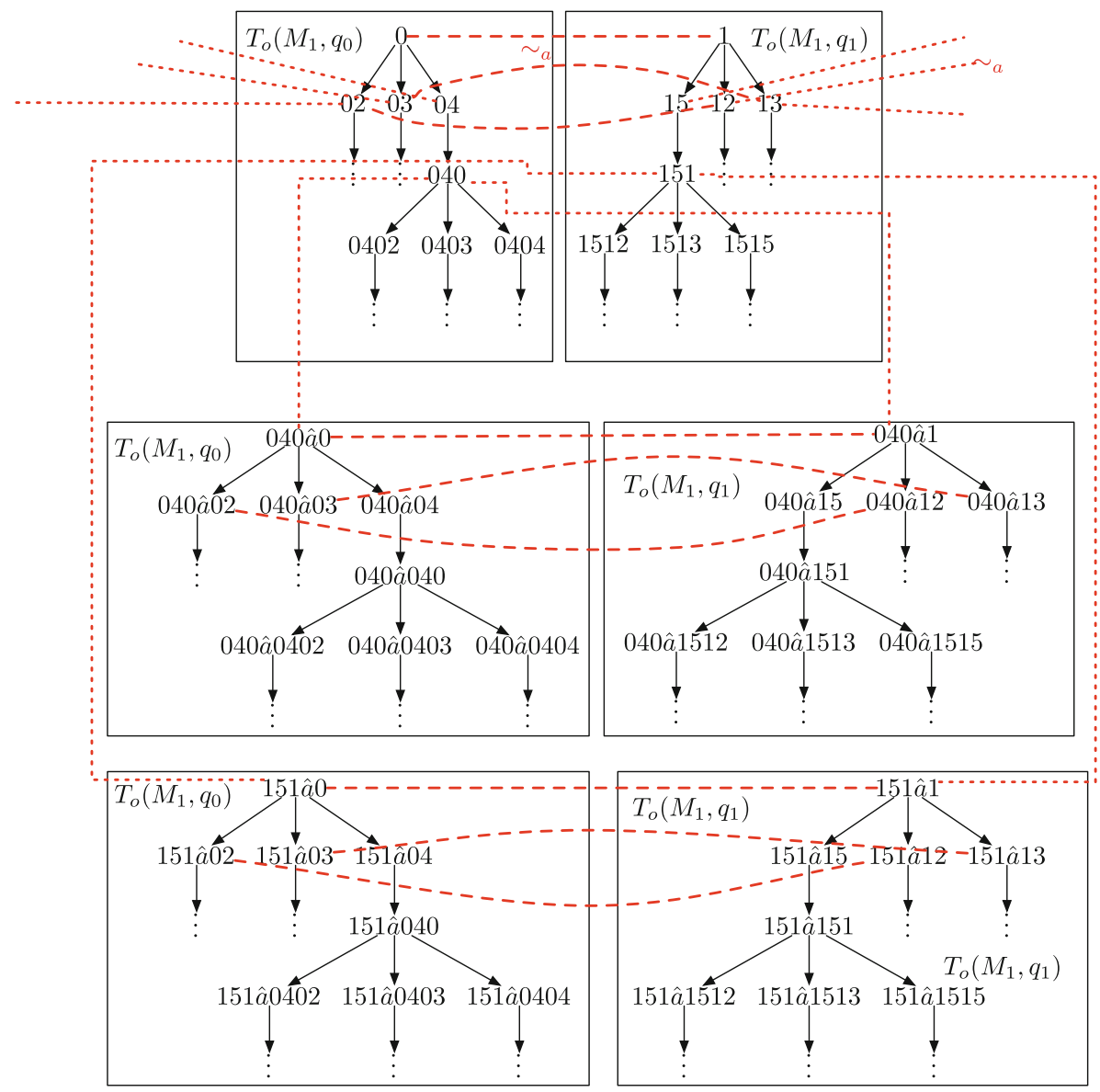

Fig. 8 The figure shows the $i_{s} R$-pando unfolding of $\left(M_{1}, q_{0}\right)$ from Fig. 5. All dotted and dashed lines denote the indistinguishability relation of agent $a$. Dashed links encode indistinguishability between nodes in trees that have roots on the same level; dotted links connect nodes in trees from different levels. Again, for the sake of readability, reflexive and transitive connections are omitted

starting in $q^{\prime}$ (cf. Fig. 8 and take e.g. $h=04, q=0$, and $q^{\prime}=040 \hat{a} 1$; the new node is named $040 \hat{a} 1$ to ensure that the name is unique as explained below). It is easy to see that these "new" subtrees can only be reached if a formula $\langle\langle a\rangle\rangle \gamma$ is evaluated in $h q$ (or some other state $h^{\prime \prime} q^{\prime \prime}$ with $h q \sim_{a}^{T_{S}\left(\mathfrak{M}, q_{0}\right)} h^{\prime \prime} q^{\prime \prime}$ by transitivity). As mentioned above all nodes in these new subtrees must have unique names. This is the reason why we have to prefix each node $h^{\prime \prime}$ in the new tree by $h q \hat{a}$ where $h q$ is the history in the "current tree" and $\hat{a}$ encodes that we have used $a$ 's indistinguishability relation to reach the "new" tree.

Before we formally define the $i_{S} R$-pando unfolding, we introduce some additional notation. In the following, we consider words over $D:=(S t \cup S t \circ\{\hat{a} \mid a \in \mathbb{A g t}\} \circ S t)^{+}$. Thus, $D$ consists of finite sequences of states, possibly interleaved by references to some agents. We use elements from $D$ to give names to nodes of the pando unfolding. Essentially, the name of a node shows how the node is reached from a root by following temporal paths and "jumping" between different trees by use of epistemic links (cf. Fig. 8). 
We also define auxiliary functions rel $: D \rightarrow S t^{+}$, ref $: D \rightarrow D$, lastr $: D \rightarrow S t$ and jump : $D \rightarrow \mathbb{A g t} \cup\{\epsilon\}$ as follows:

$$
\begin{aligned}
& \operatorname{rel}(h)= \begin{cases}h, & \text { for } h \in S t^{+} ; \\
h^{\prime \prime}, & \text { for } h=h^{\prime} \hat{a} h^{\prime \prime} \text { and } h^{\prime \prime} \in S t^{+} \text {and } a \in \mathbb{A g t} ;\end{cases} \\
& \operatorname{ref}(h)= \begin{cases}h & \text { if } h \in S t^{+} \\
\hat{h} & \text { if } h=\hat{h} \hat{a r e l}(h)\end{cases} \\
& \operatorname{lastr}(h)=\operatorname{last}(\operatorname{rel}(h)) \text {; } \\
& \operatorname{jump}(h)= \begin{cases}\epsilon & \text { if } h \in S t^{+} \\
a & \text { if } h=\hat{h} \hat{a r e l}(h) .\end{cases}
\end{aligned}
$$

The intuition for these functions is as follows. Given an element $h \in D, \operatorname{rel}(h)$ returns the "relevant" part of $h$, i.e., the subhistory at the end of $h$ of maximal length that does not contain any $\hat{a}$ symbol for any $a \in \mathbb{A g t}$. On the other hand, $r$ ef $(h)$ returns the "reference" node in the higher-level tree which was used to obtain $h$. Finally, jump $(h)$ returns the agent whose epistemic link was used to "jump" between the two trees. For example, $\operatorname{rel}\left(q_{1} \hat{a} q_{2} q_{3} \hat{b} q_{4}\right)=q_{4}$, $\operatorname{ref}\left(q_{1} \hat{a} q_{2} q_{3} \hat{b} q_{4}\right)=q_{1} \hat{a} q_{2} q_{3}, \operatorname{lastr}\left(\operatorname{ref}\left(q_{1} \hat{a} q_{2} q_{3} \hat{b} q_{4}\right)\right)=q_{3}$ and jump $\left(q_{1} \hat{a} q_{2} q_{3} \hat{b} q_{4}\right)=b$.

Let $\mathfrak{M}=\left(\mathbb{A g t}, S t, \Pi, \pi,\left\{\sim_{a}\right\}_{a \in \mathbb{A g t}}\right.$, Act $\left., d, o\right)$ be an iCGS and $q \in$ St. We recursively define sets $\Delta_{\mathfrak{M}}^{i} \subseteq D$ which contain the nodes of the $i_{S} R$-pando unfolding:

$$
\begin{aligned}
\Delta_{\mathfrak{M}}^{0}(q):= & \bigcup_{q^{\prime} \sim \mathfrak{M} q} \Lambda_{\mathfrak{M}}^{f i n}\left(q^{\prime}\right), \\
\Delta_{\mathfrak{M}}^{i+1}(q):= & \left\{h \hat{a ̂} h^{\prime}\left|h \in \Delta_{\mathfrak{M}}^{i}(q),\right| \operatorname{rel}(h) \mid \geq 2, a \in \mathbb{A g t}, \text { and } h^{\prime} \in \Lambda_{\mathfrak{M}}^{f i n}\left(q^{\prime}\right)\right. \\
& \text { for some } \left.q^{\prime} \underset{a}{\sim \mathfrak{M}} \operatorname{lastr}(h)\right\} .
\end{aligned}
$$

We write $\Delta_{\mathfrak{M}}^{i}$ for $\Delta_{\mathfrak{M}}^{i}(q)$ if state $q$ is clear from context. Note that each $h \in \Delta_{\mathfrak{M}}^{i}$ contains exactly $i$ symbols of type $\hat{a}_{j}$ for $a_{j} \in \mathbb{A g t}$ and $j=1, \ldots, i$. Intuitively, $\hat{a}$ denotes that we took $\mathrm{a} \sim \sim_{a}$-relation step between different trees. Note also that, for instance, $q_{0} q_{1} \hat{a} q_{2} \in \Delta^{1}\left(q_{0}\right)$ but $q_{0} \hat{a} q_{2} \notin \Delta^{1}\left(q_{0}\right)$. This is because if a link to a new tree model is taken histories have to be "forgotten" and in cases in which the history consists of a single state (e.g. $q_{0}$ in $q_{0} \hat{a} q_{2}$ ) such a link is not necessary and also not desired due to technical reasons. Now, we are ready to define the $i_{S} R$-pando unfolding.

Definition 17 ( $i_{S} R$-pando unfolding) Let $\mathfrak{M}=\left(\mathbb{A g t}, S t, \Pi, \pi,\left\{\sim_{a}\right\}_{a \in \mathbb{A g t}}, A c t, d, o\right)$ be an iCGS and $q \in S t$. The $i_{s} R$-pando unfolding of $(\mathfrak{M}, q)$, denoted $T_{S}(\mathfrak{M}, q)$, is defined as $T_{s}:=T_{s}(\mathfrak{M}, q)=\left(\mathbb{A g t}, S t^{\prime}, \Pi, \pi^{\prime},\left\{\sim_{a}^{\prime}\right\}_{a \in \mathbb{A g t}}, A c t, d^{\prime}, o^{\prime}\right)$ where $d^{\prime}(a, h), o^{\prime}(h, \boldsymbol{\alpha})$, and $\pi^{\prime}(h)$ are given as in Definition 12 and 15 where function "last" is replaced with "lastr" and (note that $\sim_{a}^{T_{s}}$ refers to relation $\sim_{a}^{\prime}$ ):

1. $S t^{\prime}:=\bigcup_{i=0}^{\infty} \Delta_{\mathfrak{M}}^{i}(q)$;

2. for all $a \in \mathbb{A g t}, \sim_{a}^{T_{S}} \subseteq S t_{\mathfrak{M}} \times S t_{\mathfrak{M}}$ is the smallest reflexive relation such that $h \sim_{a}^{T_{s}} h^{\prime}$ if:

(a) $\operatorname{rel}(h) \underset{a}{\mathfrak{M}} \operatorname{rel}\left(h^{\prime}\right)$, for $h, h^{\prime} \in \Delta_{\mathfrak{M}}^{0}(q)$, or

(b) $\operatorname{rel}(h) \underset{a}{\mathfrak{M}} \operatorname{rel}\left(h^{\prime}\right)$ and

i. $\operatorname{ref}(h) \sim_{a}^{T_{s}} \operatorname{ref}\left(h^{\prime}\right)$ and jump $(h)=a=j u m p\left(h^{\prime}\right)$, and $h, h^{\prime} \in \Delta_{\mathfrak{M}}^{i}(q), i>0$, or

ii. jump $(h)=b=j \operatorname{jump}\left(h^{\prime}\right)$ with $a \neq b$, and $h, h^{\prime} \in \Delta_{\mathfrak{M}}^{i}(q), i>0$, or 


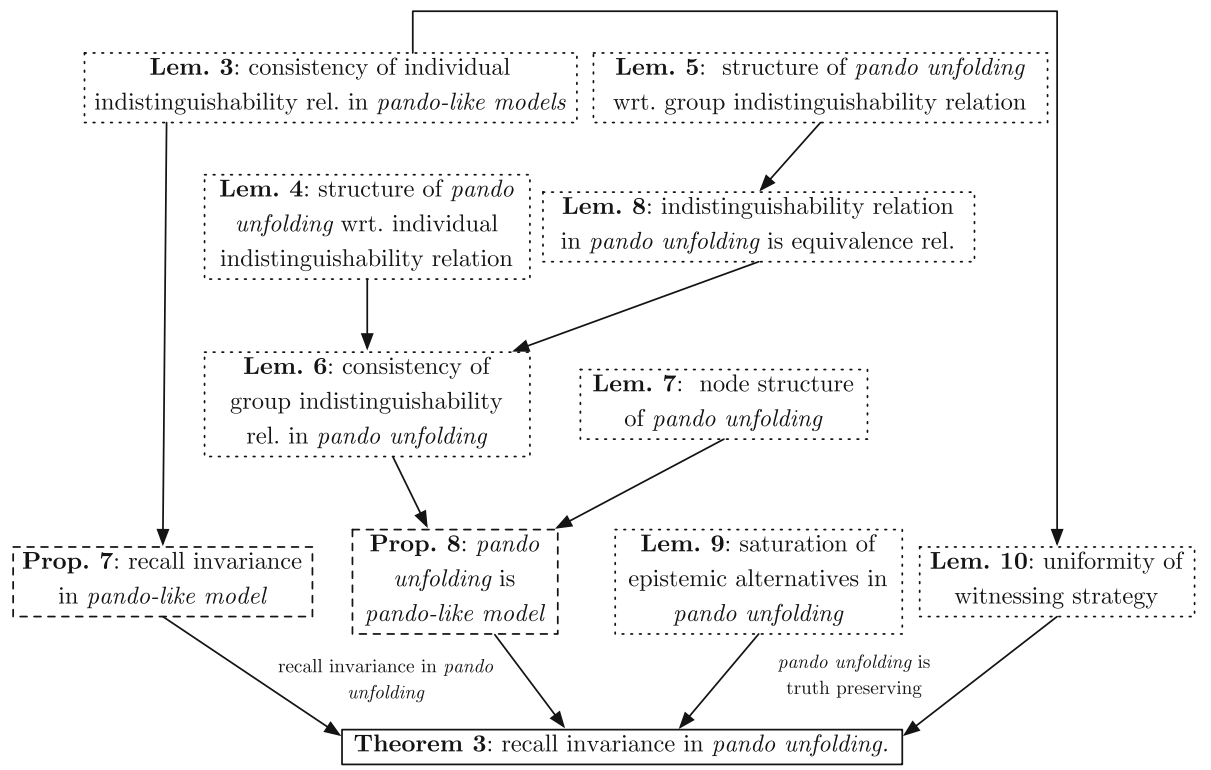

Fig. 9 Structure of the proofs of Propositions 7 and 8 and of Theorem 3. Full proofs of all results are given in Appendix 7.2

(c) $h \in \Delta_{\mathfrak{M}}^{i}, h^{\prime} \in \Delta_{\mathfrak{M}}^{i+1}, j u m p\left(h^{\prime}\right)=a, \operatorname{ref}\left(h^{\prime}\right) \sim_{a}^{T_{s}} h$, lastr $\left(\operatorname{ref}\left(h^{\prime}\right)\right) \sim_{a}^{\mathfrak{M}} \operatorname{rel}\left(h^{\prime}\right)$ or vice versa with the roles of $h$ and $h^{\prime}$ switched.

We note that this means that $h^{\prime}=\hat{h} \hat{a} q, \operatorname{lastr}(\hat{h}) \underset{a}{\sim \mathfrak{M}} q$, and $\hat{h} \sim_{a}^{T_{S}} h$ for some $q \in S t_{\mathfrak{M}}$ and $\hat{h} \in \Delta_{\mathfrak{M}}^{i}(q)$.

Remark 2 ( $i_{s} R$-pando unfolding) We motivate points 2(a), 2(b), and 2(c) in Definition 17. Items 2(a) and (b) define indistinguishability between nodes of trees from the same set $\Delta^{i}$. In this case, the "jump" must be obtained by the same epistemic relation and the final parts of the corresponding histories in the current trees (the "relevant" parts) must be indistinguishable; moreover, the "reference" nodes (in the trees one level up) must be indistinguishable for the "jump" agent (point 2(b)i) in case we are concerned with epistemic alternatives of this very agent. This is needed to obtain transitivity of the epistemic relation in the resulting forest. Note that, in particular, the length of the relevant subhistories must be the same.

Item 2(c) defines the only way how nodes $h$ and $h^{\prime}$ from different sets $\Delta^{i}$ and $\Delta^{j}, i \neq j$, can be linked via an epistemic link. Firstly, it must be the case that $j=i+1$. Secondly, the relevant part of $h^{\prime} \in \Delta^{i+1}$ must be a single state which is indistinguishable from the last state of the reference part of $h^{\prime} \in \Delta^{i}$; moreover, the reference part of $h^{\prime}$ must also be linked to $h$. Note, that the relevant parts of $h$ and $h^{\prime}$ do not have to have the same length. This models the "forgetting" if a new state-subformula is evaluated in $h$.

Example 9 ( $i_{s} R$-pando unfolding) The $i_{s} R$-pando unfolding of model $\left(M_{1}, q_{0}\right)$ from Fig. 5 is shown in Fig. 8.

Similarly to Sect. 4.2 we can show that an $i_{S} R$-pando unfolding is $i_{S} R$-pando-like as expected. For example, it has to be shown that all nodes are disjunct, in order to obtain a tree-like structure, and that the epistemic relation $\sim_{a}^{T_{s}(\mathfrak{M}, q)}$ is an equivalence relation for each agent $a \in \mathbb{A g t}$. The proof of the following result is rather technical and is formally proven 
in the Appendix. The structure of the proof of this proposition and also of our main result, Theorem 3, is outlined in Fig. 9.

Proposition 8 The $i_{S} R$-pando unfolding of a pointed $\mathbf{i C G S}$ is $i_{S} R$-pando-like.

Then, thanks to Proposition 7, we obtain that $i_{s} R$-pando unfoldings are truth-invariant under recall. Now we can state our main result for $i_{s} R$-pando unfoldings.

Theorem 3 For every iCGS $\mathfrak{M}$, state $q$ in $\mathfrak{M}$, and $\mathbf{A T L}^{*}$-formula $\varphi$, it holds that

$$
\mathfrak{M}, q \models_{i_{S} R} \varphi \text { iff } T_{S}(\mathfrak{M}, q), q \models_{i_{s} R} \varphi \text { iff } T_{S}(\mathfrak{M}, q), q \models_{i_{s} r} \varphi .
$$

Again, the proof is moved to the Appendix.

\section{Comparing validities for variants of ATL}

In this section we present a formal comparison of the semantic variants defined in Sects. 2 and 3. As stated in the introduction, we compare the variants on the level of their validity sets (or, equivalently, satisfiable sentences). In most cases, they turn out to be different. Also, we can usually show that one variant is a refinement of the other in the sense that its set of validities strictly subsumes the validities induced by the other variant.

In what follows, we write $\operatorname{Val}\left(\mathbf{A T L} \mathbf{L}_{\text {sem }}\right)$ to denote the set of $\boldsymbol{A T L}$ validities, or the theory of $\boldsymbol{A T L}$, under semantics sem. Likewise, we write Sat $\left(\mathbf{A T L} \mathbf{L}_{\text {sem }}\right)$ for the set of ATL formulae satisfiable in the semantics sem. Note that validity and satisfiability of formulae in all cases considered in this paper is defined over the same class of models, namely the class of imperfect information concurrent game structures. The conceptual reading of $\operatorname{Val}\left(\mathbf{A T L}_{\text {sem }_{1}}\right) \subsetneq \operatorname{Val}\left(\mathbf{A} \mathbf{T L} \mathbf{s e m}_{2}\right)$ can be as follows: for "game boards" given by iCGS's, we have that the "game rules" in the $\mathbf{A} \mathbf{T L} \mathbf{s e m}_{1}$ variant strictly refine the rules in $\mathbf{A} \mathbf{T L} \mathbf{s e m}_{2}$. Note also that $\operatorname{Val}\left(\mathbf{A} \mathbf{T} \mathbf{L}_{\text {sem }_{1}}\right) \subsetneq \operatorname{Val}\left(\mathbf{A T L} \mathbf{s e m}_{2}\right)$ is equivalent to $\operatorname{Sat}\left(\mathbf{A} \mathbf{T L} \mathbf{s e m}_{2}\right) \subsetneq \operatorname{Sat}\left(\mathbf{A T L} \mathbf{s e m}_{1}\right)$. Thus, an alternative reading is " $\mathbf{A} \mathbf{T L}_{\text {sem }_{1}}$ admits reasoning about a larger variety of games than $\mathbf{A T L}_{\text {sem }_{2}}$ ".

We will always prove inclusion results for the broadest possible language (usually ATL ${ }^{*}$ ) and non-inclusion results for the narrowest one (usually ATL). Clearly, for languages $\mathcal{L} \subseteq \mathcal{L}^{\prime}$, we have that $\operatorname{Val}\left(\mathcal{L}_{\text {sem }}^{\prime}\right) \subseteq \operatorname{Val}\left(\mathcal{L}_{\text {sem }}^{\prime}\right)$ implies $\operatorname{Val}\left(\mathcal{L}_{\text {sem }_{1}}\right) \subseteq \operatorname{Val}\left(\mathcal{L}_{\text {sem }_{2}}\right)$, and $\operatorname{Val}\left(\mathcal{L}_{\text {sem }_{1}}\right) \not \mathbb{E}$ $\operatorname{Val}\left(\mathcal{L}_{\text {sem }_{2}}\right)$ implies $\operatorname{Val}\left(\mathcal{L}_{\text {sem }}^{\prime}\right) \not \operatorname{Val}\left(\mathcal{L}_{\text {sem }}^{\prime}\right)$

Summary of the results Figure 10 gives an overview of the results of Sects. 5.3-5.6. We show that almost all the semantic variants discussed here are different on the level of validities, and that they show a strong pattern: perfect information is a special case of imperfect information, perfect recall games are special case of memoryless games, and properties of objective and subjective abilities of agents are incomparable. Moreover, the type of information has more impact on the validities than the type of recall in the more restricted language of ATL. Interestingly, for the richer languages of $\mathbf{A T L}^{+}$and $\mathbf{A T L}{ }^{*}$ this is not the case anymore.

Note that if we reverse the subsumption signs in Fig. 10 then the graphs describe the hierarchy of satisfiable sentences in different semantics of ATL/ATL*.

Remark 3 It is important to observe that comparing validities is not the same as comparing abilities. For example, subjective ability to enforce $\gamma$ always implies objective ability to enforce $\gamma$. Yet, as we show in Sect. 5.6, the set of validities for objective ability does not subsume the one for subjective ability. It is tempting to think that it should, because for every validity $\langle\langle A\rangle\rangle \gamma$ in the subjective semantics, $\langle\langle A\rangle\rangle \gamma$ must be also valid in the objective 
(A)

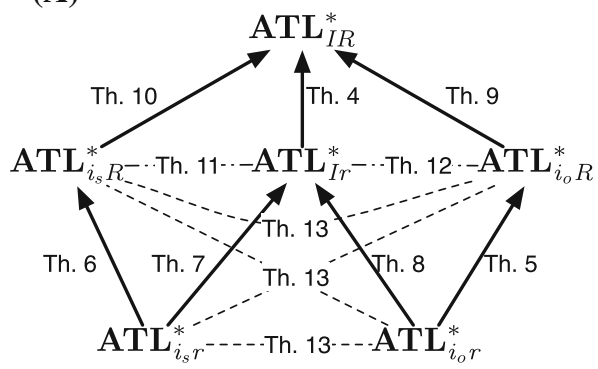

(B)

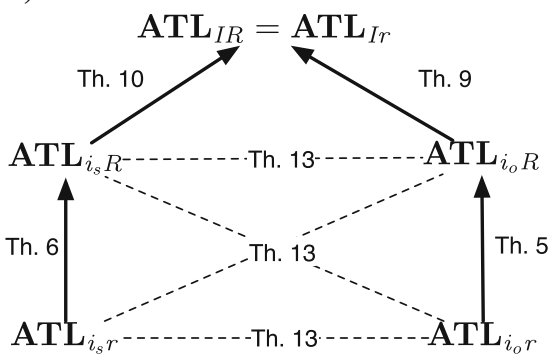

Fig. 10 Comparison of validity sets induced by various semantics of a ATL*, and b ATL. Arrows depict strict subsumption of validity sets, e.g., "ATL $\mathbf{I r}^{*} \rightarrow \mathbf{A T L} \mathbf{I R}_{I R}^{*}$ means that $\operatorname{Val}\left(\mathbf{A} \mathbf{T L}_{I r}^{*}\right) \subsetneq \operatorname{Val}\left(\mathbf{A T L} \mathbf{I R}^{*}\right)$. Dotted lines connect semantic variants with incomparable validity sets. We do not include links that follow from transitivity of the subsumption relation. The hierarchy for $\mathbf{A T L} \mathbf{L}^{+}$is exactly the same as for $\mathbf{A T L} \mathbf{L}^{*}$

semantics. On the other hand, what about validities stating inability, i.e., $\neg\langle\langle A\rangle\rangle \gamma$ ? Should they adhere to the reverse subsumption? Either way, this line of reasoning is totally misleading.

The reason for that is simple. Almost no formulae of type $\langle\langle A\rangle\rangle \gamma$ or $\neg\langle\langle A\rangle\rangle \gamma$ are validities of ATL in any semantics that we study. There are only two exceptions: $\langle\langle A\rangle\rangle T$ and $\neg\langle\langle A\rangle \perp$. Or, to be more precise, all formulae $\langle\langle A\rangle\rangle \gamma$ where $\gamma$ is tautologically true (i.e., holds on all paths that can occur in any CGS) and $\neg\langle\langle A\rangle\rangle \gamma$ where $\gamma$ is tautologically false (i.e., fails on all paths in all CGS's). For a nontrivial ability (that is, one which refers to a temporal property that can, but does not have to be true), a valid formula can only connect it to another kind of ability. For example, $\langle\langle A\rangle\rangle p \rightarrow\langle\langle A \cup B\rangle\rangle p$ is valid in all the semantics considered in this paper.

\subsection{Perfect recall versus memoryless play under perfect information (IR vs. Ir)}

We first proceed to examine the impact of recall on the general strategic properties of agent systems under prefect information. The inclusion results follow naturally from the invariance theorems for tree-like unfoldings presented in Sect. 4. Non-inclusion will be demonstrated by appropriate formulae (that are valid in one semantics and not valid in another). We have already mentioned that, in ATL, the $I r$ - and $I R$-semantics coincide (Proposition 1). As a consequence, they induce the same validities: $\operatorname{Val}\left(\mathbf{A T L}_{I r}\right)=\operatorname{Val}\left(\mathbf{A T L}_{I R}\right)$. Thus, regardless of the type of their recall, perfect information agents possess the same abilities with respect to winning conditions that can be specified in ATL. An interesting question is: Does it carry over to more general classes of winning conditions, or are there (broader) languages that can discern between the two types of ability? The answer is: no, it doesn't, and yes, there are. The $I r$ - and $I R$-semantics induce different validity sets for $\mathbf{A T L}^{*}$, and in fact the distinction is already present in $\mathbf{A} \mathbf{T L}{ }^{+}$. Moreover, it turns out that perfect recall can be seen as a special case of memoryless play in the sense of their general properties.

Proposition $9 \operatorname{Val}\left(\boldsymbol{A T L} L_{I r}^{*}\right) \subseteq \operatorname{Val}\left(\boldsymbol{A T L} L_{I R}^{*}\right)$

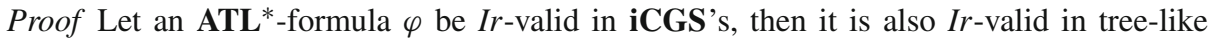
CGS's, and by Proposition 2 also $I R$-valid in tree-like CGS's. Thus, by Theorem 1, it is $I R$ valid in arbitrary CGS's. Since indistinguishability relations do not influence the semantic relation $\models_{I R}$, we get that $\varphi$ is $I R$-valid in iCGS's.

In particular, the subsumption holds for formulae of $\mathbf{A T L}^{+}$. Moreover: 
Fig. 11 Single-agent model $M_{5}$ : robot with multiple tasks

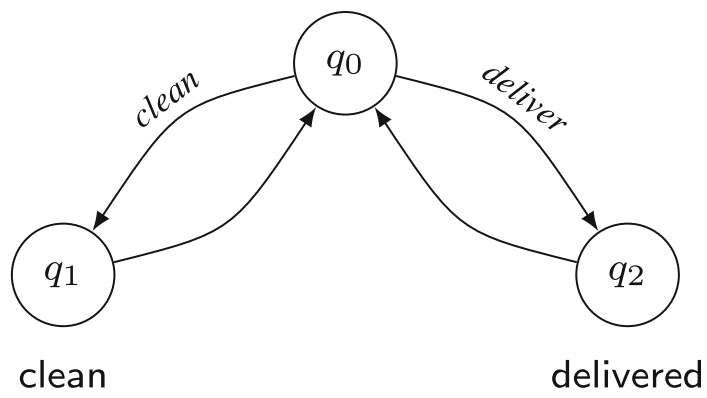

Proposition $10 \operatorname{Val}\left(\boldsymbol{A T L} L_{I R}^{+}\right) \nsubseteq \operatorname{Val}\left(\boldsymbol{A T L}_{I r}^{+}\right)$.

Proof Consider formula

$$
\Phi_{3} \equiv\langle\langle a\rangle\rangle\left(\diamond \mathrm{p}_{1} \wedge \diamond \mathrm{p}_{2}\right) \leftrightarrow\langle\langle a\rangle\rangle \diamond\left(\mathrm { p } _ { 1 } \wedge \langle \langle a \rangle \rangle \left\langle\mathrm{p}_{2} \vee \mathrm{p}_{2} \wedge\langle\langle a\rangle\rangle\left\langle\mathrm{p}_{1}\right) .\right.\right.
$$

The formula is valid in $\mathbf{A T L}_{I R}^{+}$[33]. On the other hand, its right-to-left part is not valid in $\mathbf{A T L}_{I r}^{+}$. To see this, we take the single-agent CGS $M_{5}$ from Fig. 11 where agent $a$ (the robot) can do the cleaning or deliver a package. Then, for $\mathrm{p}_{1} \equiv$ clean, $\mathrm{p}_{2} \equiv$ delivered, we have $M_{5}, q_{0} \models_{I r}\langle\langle a\rangle\rangle \diamond\left(\mathrm{p}_{1} \wedge\langle\langle a\rangle\rangle \diamond \mathrm{p}_{2} \vee \mathrm{p}_{2} \wedge\langle\langle a\rangle\rangle \diamond \mathrm{p}_{1}\right)$ but also $M_{5}, q_{0} \forall_{I r}\langle\langle a\rangle\rangle\left(\diamond \mathrm{p}_{1} \wedge\left\langle\mathrm{p}_{2}\right)\right.$.

Theorem $4 \operatorname{Val}\left(\boldsymbol{A T L} L_{I r}\right)=\operatorname{Val}\left(\boldsymbol{A T L} L_{I R}\right)$. However, $\operatorname{Val}\left(\boldsymbol{A T L} L_{I r}^{+}\right) \subsetneq \operatorname{Val}\left(\boldsymbol{A T L}+L_{I R}\right)$ and $\operatorname{Val}\left(\boldsymbol{A T L} \boldsymbol{L}_{I r}^{*}\right) \subsetneq \operatorname{Val}\left(\boldsymbol{A T L} \boldsymbol{L}_{I R}^{*}\right)$.

Proof From Proposition 1 it follows that $\operatorname{Val}\left(\mathbf{A} \mathbf{A L}_{I r}\right)=\operatorname{Val}\left(\mathbf{A} \mathbf{A L}_{I R}\right)$. From Proposition 9 we know that $\operatorname{Val}\left(\mathbf{A T L} \mathbf{I r}_{I r}^{*}\right) \subseteq \operatorname{Val}\left(\mathbf{A T L} \mathbf{L}_{I R}^{*}\right)$ and can also deduce that $\operatorname{Val}\left(\mathbf{A T L} \mathbf{I I}_{I r}^{+}\right) \subseteq \operatorname{Val}\left(\mathbf{A T L} \mathbf{T L}_{I R}^{+}\right)$ because the language of $\mathbf{A T L}^{+}$is just a syntactic restriction of the one of $\mathbf{A T L}^{*}$. Finally, Proposition 10 proves that $\operatorname{Val}\left(\mathbf{A T L} \mathbf{L}_{I r}^{+}\right) \subsetneq \operatorname{Val}\left(\mathbf{A} \mathbf{T L}{ }_{I R}^{+}\right)$and also that $\operatorname{Val}\left(\mathbf{A T L} \mathbf{L}_{I r}^{*}\right) \subsetneq$ $\operatorname{Val}\left(\mathbf{A T L}_{I R}^{*}\right)$ because the formula given in the proof of the very proposition is in particular also an ATL*-formula.

\subsection{Perfect recall versus memoryless play under imperfect information ( $i R$ vs. $i r)$}

Now we compare the memoryless and perfect recall semantics under uncertainty. We treat the case of objective and subjective ability separately.

\subsubsection{Imperfect information: objective ability}

Proposition $11 \operatorname{Val}\left(\boldsymbol{A T L} \boldsymbol{i}_{i_{r}}^{*}\right) \subseteq \operatorname{Val}\left(\boldsymbol{A T L} L_{i_{o} R}^{*}\right)$.

Proof We prove that $\operatorname{Sat}\left(\mathbf{A T L} \mathbf{i}_{i_{0} R}^{*}\right) \subseteq \operatorname{Sat}\left(\mathbf{A T L}_{i_{o} r}^{*}\right)$. Let $\varphi \in \operatorname{Sat}\left(\mathbf{A T L}_{i_{o} R}^{*}\right)$. Then, there must be a pointed iCGS $(\mathfrak{M}, q)$ such that $\mathfrak{M}, q \models_{i_{o} R} \varphi$. By Theorem 2, $T_{o}(\mathfrak{M}, q), q \models_{i_{o} R}$ $\varphi$. But on $i_{o} R$-tree unfoldings, $i R$ - and $i r$-strategies coincide (Theorem 2), so we get that $T_{o}(\mathfrak{M}, q), q \models_{i_{o} r} \varphi$, and as a consequence $\varphi \in \operatorname{Sat}\left(\mathbf{A T L}_{i_{o} r}^{*}\right)$.

The converse does not hold:

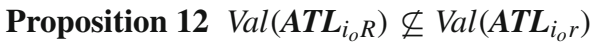


Fig. 12 Model $M_{6}$ with $\mathbb{A g t}=\{a\}$ : dangers of marital life

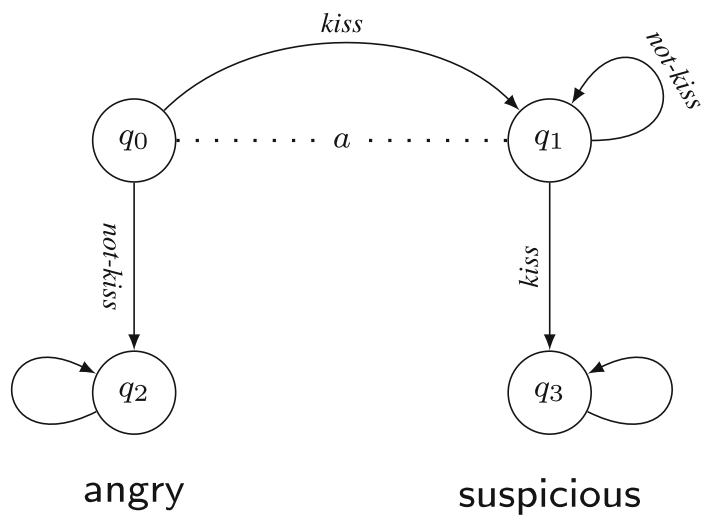

Proof To show this, we take the ATL embedding of the CTL duality between combinators $\mathrm{E} \square$ and $\mathrm{A} \diamond$ (see Sect. 2.5). In fact, only one direction of the equivalence is important here:

$$
\Phi_{4} \equiv \neg\langle\langle\emptyset\rangle\rangle \neg \mathrm{p} \rightarrow\langle\langle\mathbb{A g t}\rangle\rangle \square \mathrm{p}
$$

(note that the other direction is valid for all the semantics considered in this paper, and actually for all the reasonable semantics of strategic ability that one can come up with).

First, we observe that: (i) $\neg\langle\langle\emptyset\rangle\rangle \diamond \neg p$ expresses (regardless of the actual type of ability being considered) that there is a path in the system on which $p$ always holds; (ii) in the "objective" semantics the set $\operatorname{out}\left(q, s_{\mathrm{Agt}}\right)$ always consists of exactly one path; (iii) for every path $\lambda$ starting from $q$, there is an $i_{o} R$-strategy $s_{\mathbb{A g t}} \operatorname{such}$ that out $\left(q, s_{\mathbb{A g t}}\right)=\{\lambda\}$. From these, it is easy to see that $\Phi_{4}$ is valid in $\mathbf{A T L} \mathbf{L}_{i_{0} R}$.

Second, we consider model $M_{6}$ in Fig. $12 .{ }^{10}$ Let us take $\mathrm{p} \equiv \neg$ angry $\wedge \neg$ suspicious. Then, we have $M_{6}, q_{0} \models_{i_{o} r} \neg\langle\langle\emptyset\rangle\rangle \diamond \neg \mathrm{p}$ but also $M_{6}, q_{0} \nvdash_{i_{o} r}\langle\langle\mathrm{Agt}\rangle\rangle \square \mathrm{p}$, which demonstrates that $\Phi_{4}$ is not valid in $\mathbf{A T L}_{i_{o} r}$.

Theorem $5 \operatorname{Val}\left(\boldsymbol{A T L} \boldsymbol{i}_{i}\right) \subsetneq \operatorname{Val}\left(\boldsymbol{A} \boldsymbol{T L} \boldsymbol{i}_{i_{0}}\right)$, and similarly for $\boldsymbol{A T L}^{+}$and $\boldsymbol{A T L} \mathbf{L}^{*}$.

\subsubsection{Imperfect information: subjective ability}

Proposition $13 \operatorname{Val}\left(\boldsymbol{A T L} L_{i_{s} r}^{*}\right) \subseteq \operatorname{Val}\left(\boldsymbol{A T L}_{i_{s} R}^{*}\right)$.

Proof Analogous to Proposition 11.

Proposition $14 \operatorname{Val}\left(\boldsymbol{A T L}_{i_{s} R}\right) \nsubseteq \operatorname{Val}\left(\boldsymbol{A T L} L_{i_{s}}\right)$.

Proof We take the formula $\Phi_{5}$ which is a consequence of the fixpoint equivalence for $\langle\langle a\rangle\rangle\langle\mathrm{p}$ :

$$
\Phi_{5} \equiv\langle\langle a\rangle\rangle \bigcirc\langle\langle a\rangle\rangle \vee \mathrm{p} \rightarrow\langle\langle a\rangle\rangle \vee \mathrm{p} .
$$

The formula states that if $a$ has an opening move and a follow-up strategy to achieve $p$ eventually, then these can be integrated into a single strategy achieving $p$ already from the

10 The example depicts some simple traps that await a married man if he happens to be absent-minded. If he doesn't kiss his wife in the morning, he is likely to make her angry. However, if he kisses her more than once, she might get suspicious. It is easy to see that the absent-minded (i.e., memoryless) husband does not have a strategy to survive safely through the morning, though a safe path through the model does exist $\left(\lambda=q_{0} q_{1} q_{1} q_{1} \ldots\right)$. 
initial state. It is easy to see that $\Phi_{5}$ is valid in $\mathbf{A T L}_{i_{s} R}$, and that the single strategy is just a concatenation of the two strategies that we get on the left hand side of the implication. On the other hand, for the "poor duck model" $M_{1}$ and $\mathrm{p} \equiv$ shot, we get that $M_{1}, q_{0} \models_{i_{s} r}$ $\langle\langle a\rangle\rangle \bigcirc\langle\langle a\rangle\rangle \vee \mathrm{p}$ but also $M_{1}, q_{0} \not \models_{i_{s} r}\langle\langle a\rangle\rangle \vee \mathrm{p}$, so $\Phi_{5}$ is not valid in $\mathbf{A T L} \mathbf{L}_{i_{s} r}$.

Theorem $6 \operatorname{Val}\left(\boldsymbol{A T L _ { i _ { s } } )} \subsetneq \operatorname{Val}\left(\boldsymbol{A} \boldsymbol{T L} \boldsymbol{i}_{s} R\right)\right.$, and similarly for $\boldsymbol{A T L}^{+}$and $\boldsymbol{A T L} \mathbf{L}^{*}$.

\subsection{Perfect versus imperfect information under memoryless play (Ir vs. ir)}

We continue by comparing perfect and imperfect information scenarios. That is, in the first class $(I)$, agents recognize the current global state of the system by definition. In the latter $\left(i_{s} / i_{o}\right)$, uncertainty of agents about states constrains their choices. Firstly, we observe that perfect information can be seen as a special case of imperfect information.

Proposition $15 \operatorname{Val}\left(\boldsymbol{A T L} L_{i_{s} r}^{*}\right) \subseteq \operatorname{Val}\left(\boldsymbol{A T L} L_{I r}^{*}\right)$ and $\operatorname{Val}\left(\boldsymbol{A T L} L_{i_{o} r}^{*}\right) \subseteq \operatorname{Val}\left(\boldsymbol{A T L} L_{I r}^{*}\right)$.

Proof Since perfect information of agents can be explicitly represented in iCGS by fixing all relations $\sim_{a}$ as the minimal reflexive relations $\left(q \sim_{a} q^{\prime}\right.$ iff $\left.q=q^{\prime}\right)$, we have that $\varphi \in$ $\operatorname{Sat}\left(\mathbf{A T L} \mathbf{I}_{I r}^{*}\right)$ implies $\varphi \in \operatorname{Sat}\left(\mathbf{A T L} \mathbf{i}_{s}^{*}\right)$ and $\varphi \in \operatorname{Sat}\left(\mathbf{A T L} i_{i_{o} r}^{*}\right)$. Thus, dually, $\operatorname{Val}\left(\mathbf{A T L} \mathbf{L}_{i_{r} r}^{*}\right) \subseteq$ $\operatorname{Val}\left(\mathbf{A T L} \mathbf{I r}^{*}\right)$ and $\operatorname{Val}\left(\mathbf{A} \mathbf{T L} i_{i_{o} r}^{*}\right) \subseteq \operatorname{Val}\left(\mathbf{A T L} \mathbf{I r}_{I r}^{*}\right)$.

Proposition $16 \operatorname{Val}\left(\boldsymbol{A T L} L_{I r}\right) \nsubseteq \operatorname{Val}\left(\boldsymbol{A T L} \boldsymbol{i}_{i_{r}}\right)$.

Proof We show this by presenting a validity for $\mathbf{A T L} \mathbf{L}_{I r}$ which is not valid in $\mathbf{A T L} \mathbf{L}_{i_{s}}$. Consider the formula that captures the right-to-left direction in the fixpoint characterization of $\langle\langle A\rangle\rangle \diamond \varphi$ for single-agent teams and atomic propositions:

$$
\Phi_{1} \equiv(\mathrm{p} \vee\langle\langle a\rangle\rangle \bigcirc\langle\langle a\rangle\rangle \vee \mathrm{p}) \rightarrow\langle\langle a\rangle\rangle \vee \mathrm{p}
$$

$\Phi_{1}$ is $I r$-valid (cf. Sect. 2.5). To see its invalidity in the $i_{S} r$-semantics, consider model $M_{1}$ from Fig. 5. We recall that the story behind the model is as follows. A man wants to shoot down a yellow rubber duck in a shooting gallery. The man knows that the duck is in one of the two cells in front of him, but he does not know in which one. Moreover, this has been a long party, and he is very tired, so he is only capable of using memoryless strategies at the moment. Does he have a memoryless strategy which he knows will achieve the goal? No. He can either decide to shoot to the left, or to the right, or reach out to the cells and look what is in (note also that the cells close in the moment after being opened). In each of these cases the man risks that he will fail (at least from his subjective point of view). Can he identify an opening strategy that will guarantee his knowing how to shoot the duck in the next moment? Yes. The opening strategy is to look; if the system proceeds to $q_{4}$ then the second strategy is to shoot to the left, otherwise the second strategy is to shoot to the right.

Indeed, for $\mathrm{p} \equiv$ shot, we get $M_{1}, q_{0} \models_{i_{s} r} \mathrm{p} \vee\langle\langle a\rangle\rangle \bigcirc\langle\langle a\rangle\rangle\left\langle\mathrm{p}\right.$ and $M_{1}, q_{0} \nvdash_{i_{s} r}\langle\langle a\rangle\rangle\langle\mathrm{p}$, which formally concludes our proof.

\section{Proposition $17 \operatorname{Val}\left(\boldsymbol{A T L} \boldsymbol{L}_{I r}\right) \nsubseteq \operatorname{Val}\left(\boldsymbol{A T L _ { i _ { o } } )}\right)$}

Proof It is sufficient to show that $\Phi_{1} \equiv(\mathrm{p} \vee\langle\langle a\rangle\rangle \bigcirc\langle\langle a\rangle\rangle\langle\mathrm{p}) \rightarrow\langle\langle a\rangle\rangle \diamond \mathrm{p}$ is invalid in the $i_{o} r$-semantics. Take model $M_{2}$ in Fig. 13 and $\mathrm{p} \equiv$ shot. Now we have that $M_{2}, q_{0}^{\prime} \models_{i_{o} r}$ $\mathrm{p} \vee\langle\langle a\rangle\rangle \bigcirc\langle\langle a\rangle\rangle\left\langle\mathrm{p}\right.$ because $a$ has a uniform strategy that objectively achieves $\diamond \mathrm{p}$ in $q_{0}$ $\left(s_{a}(q)=\operatorname{shoot}_{L}\right.$ for every $\left.q\right)$ and another uniform strategy in $q_{1}\left(s_{a}^{\prime}(q)=\operatorname{shoot}_{R}\right.$ for every $q$ ). However, $s_{a}$ and $s_{a}^{\prime}$ cannot be merged into a single uniform strategy, and indeed $M_{2}, q_{0}^{\prime} \not{ }_{i_{o} r}\langle\langle a\rangle\rangle \diamond \mathrm{p}$, which concludes the proof. 


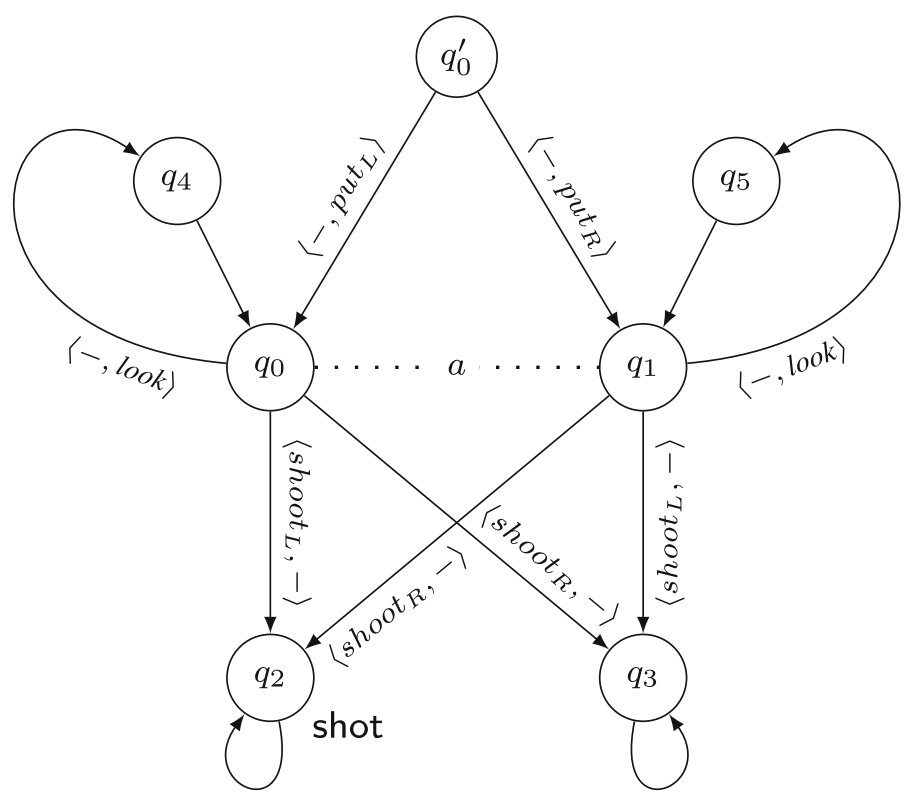

Fig. 13 Modified "poor duck" model $M_{2}$ with two agents $a, b$. This time, we explicitly represent the agent (b) who puts the duck in one of the cells

Note that, for $\mathbf{A} \mathbf{T} \mathbf{L}_{i_{o} r}$, formula $\Phi_{1}$ is valid in single-agent models, so we really needed to add another agent to the picture.

The following theorems are straightforward consequences.

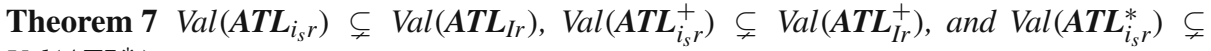
$\operatorname{Val}\left(\boldsymbol{A} \boldsymbol{T L} \boldsymbol{L}_{I r}^{*}\right)$.

Theorem $8 \operatorname{Val}\left(\boldsymbol{A T L} L_{i_{o}}\right) \subsetneq \operatorname{Val}\left(\boldsymbol{A T L} L_{I r}\right)$, and similarly for $\boldsymbol{A T L}^{+}$and $\boldsymbol{A T L}{ }^{*}$.

By Proposition 1 and Theorems 4, 7, and 8, we get the following corollary:

Corollary $1 \operatorname{Val}\left(\boldsymbol{A T L} L_{i_{s} r}\right) \subsetneq \operatorname{Val}\left(\boldsymbol{A T L} L_{I R}\right)$ and $\operatorname{Val}\left(\boldsymbol{A T L} L_{i_{0} r}\right) \subsetneq \operatorname{Val}\left(\boldsymbol{A T L} L_{I R}\right)$, and similarly for $\mathbf{A T L}^{+}$and ATL*.

5.4 Perfect versus imperfect information under perfect recall play (IR vs. $i R$ )

First, we observe that for $\mathbf{A T L}_{i_{0} R}$ versus $\mathbf{A T L} L_{I R}$ we can employ the same reasoning as for $\mathbf{A T L}_{i_{o} r}$ versus $\mathbf{A} \mathbf{T L}_{I r}$. Abilities under perfect information can be still seen as a special case of imperfect information abilities, and we can use the same model $M_{2}$ to invalidate the same formula $\Phi_{1}$ in $\mathbf{A T L}_{i_{0} R}$. Thus, analogously to Theorem 8 we get:

Theorem $9 \operatorname{Val}\left(\boldsymbol{A T L _ { i _ { 0 } } )} \subsetneq \operatorname{Val}\left(\boldsymbol{A T L} \boldsymbol{L}_{I R}\right)\right.$, and similarly for $\boldsymbol{A T L}^{+}$and $\boldsymbol{A T L} \boldsymbol{T H}^{*}$.

By the same reasoning as above, $\operatorname{Val}\left(\mathbf{A T L} i_{i_{s}}\right) \subseteq \operatorname{Val}\left(\mathbf{A T L} \mathbf{L}_{I R}\right)$. To settle the other direction, we need to use another counterexample, though.

Proposition $18 \operatorname{Val}\left(\boldsymbol{A T L} L_{I R}\right) \nsubseteq \operatorname{Val}\left(\boldsymbol{A T L}_{i_{S} R}\right)$.

Proof This time we consider the other direction of the fixpoint characterization for $\langle\langle a\rangle\rangle \mathrm{p}$ : 
Fig. 14 Variant of "poor duck" after a particularly long party (model $M_{3}$ )

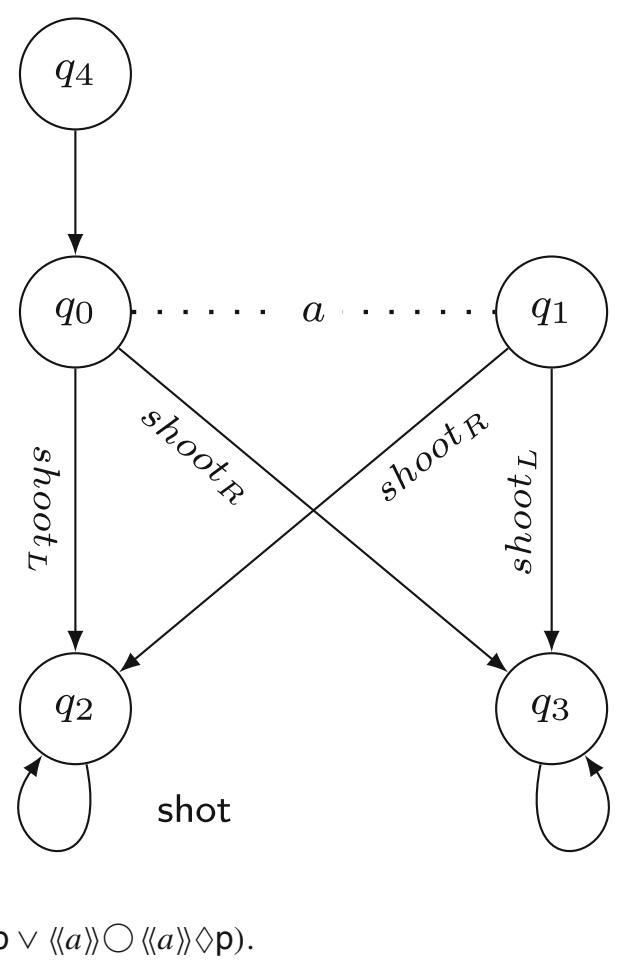

$\Phi_{2}$ is $I R$-valid, but it is not valid in $i_{S} R$. Consider a modification of the "poor duck model" in Fig. 14 (the party goes on, and the man is not even able to reach out and look anymore; the cells are open initially but they will close in a moment). Take $p \equiv$ shot. We have that $M_{3}, q_{4} \models_{i_{s} R}\langle\langle a\rangle\rangle \diamond \mathrm{p}$, but $M_{3}, q_{4} \models_{i_{s} R} \mathrm{p} \vee\langle\langle a\rangle\rangle \bigcirc\langle\langle a\rangle\rangle \diamond \mathrm{p}$, which concludes the proof.

Theorem $10 \operatorname{Val}\left(\boldsymbol{A T L} \boldsymbol{i}_{s}\right) \subsetneq \operatorname{Val}\left(\boldsymbol{A T L} L_{I R}\right)$, and similarly for $\boldsymbol{A T L}^{+}$and $\boldsymbol{A T L} \boldsymbol{L}^{*}$.

5.5 Mixed setting: information versus memory ( $I r$ vs. $i R$ )

In this section we compare abilities if both dimensions change. For ATL we already know the complete picture because $\mathbf{A T L}_{I r}$ and $\mathbf{A T L} \mathbf{L}_{I R}$ are the same logics, cf. Fig. 10b. For ATL* it remains to compare $\mathbf{A} \mathbf{T L} \mathbf{L}_{I r}^{*}, \mathbf{A} \mathbf{T L}_{i_{o} R}^{*}$, and $\mathbf{A} \mathbf{T L}_{i_{s} r}^{*}$.

To facilitate proofs, we define an additional temporal operator $\mathrm{N}$ ("now") as $\mathrm{N} \varphi \equiv \varphi \mathcal{U} \varphi$. Note that $\mathfrak{M}, \lambda \models \mathrm{N} \varphi$ iff $\mathfrak{M}, \lambda \models \varphi$ in the semantics of $\mathbf{C T L}^{*}$ and any ATL-semantics that we have discussed in this paper. Moreover, we note that the formula $\langle\langle A\rangle\rangle \mathrm{N} \varphi$ expresses $E_{A} \varphi$ (everybody in $A$ knows that $\varphi$ ) if $\langle\langle A\rangle$ is interpreted according to the subjective semantics for imperfect information (i.e., according to $\models_{i_{s} R}$ and $\models_{i_{s} r}$ ).

Theorem 11 The sets $\operatorname{Val}\left(\boldsymbol{A T L} L_{i_{s} R}^{*}\right)$ and $\operatorname{Val}\left(\boldsymbol{A T L} L_{I r}^{*}\right)$ are incomparable, and similarly for $\mathrm{ATL}^{+}$.

Proof We prove incomparability for $\mathbf{A T L}^{+}$. From this, incomparability for $\mathbf{A T L}^{*}$ follows immediately.

1. $\operatorname{Val}\left(\mathbf{A} \mathbf{T L} \mathbf{L}_{I r}^{*}\right) \nsubseteq \operatorname{Val}\left(\mathbf{A} \mathbf{T L}_{i_{s} R}^{*}\right)$. Suppose that $\operatorname{Val}\left(\mathbf{A} \mathbf{T L} L_{I r}^{*}\right) \subseteq \operatorname{Val}\left(\mathbf{A} \mathbf{T L} i_{i_{S} R}^{*}\right)$. This implies that $\operatorname{Val}\left(\mathbf{A T L} \mathbf{L}_{I r}\right) \subseteq \operatorname{Val}\left(\mathbf{A T L} i_{i_{s} R}\right)$ and by Theorem $4 \operatorname{Val}\left(\mathbf{A T L} \mathbf{L}_{I R}\right) \subseteq \operatorname{Val}\left(\mathbf{A T L} \mathbf{i}_{i_{S} R}\right)$. But this contradicts Theorem 10. 
2. $\operatorname{Val}\left(\mathbf{A} \mathbf{T L}_{i_{s} R}^{*}\right) \nsubseteq \operatorname{Val}\left(\mathbf{A} \mathbf{T L}_{I r}^{*}\right)$. For this case we consider the $\mathbf{A} \mathbf{T L}^{*}$-formula

$$
\Phi_{6}=\langle\langle\mathbb{A g t}\rangle\rangle \diamond\langle\langle\mathrm{Agt}\rangle\rangle \mathrm{N}\left(\mathrm{p}_{1} \wedge\langle\langle\mathbb{A g t}\rangle\rangle \mathrm{p}_{2}\right) \rightarrow\langle\langle\mathbb{A g t}\rangle\rangle\left(\diamond \mathrm{p}_{1} \wedge \diamond \mathrm{p}_{2}\right)
$$

which is a validity of $\mathbf{A} \mathbf{T L}_{i_{s} R}^{+}$but not of $\mathbf{A} \mathbf{T L}_{I r}^{+}$. The latter fact can be shown by the same counterexample as used in the proof of Proposition 10 (we have $M_{5}, q_{0} \not \nvdash_{I r} \Phi_{6}$ ).

It remains to show that $\Phi_{6} \in \operatorname{Val}\left(\mathbf{A T L}_{i_{s} R}^{+}\right)$. Suppose that $\mathfrak{M}, q \models_{i_{s} R}\langle\langle\mathbb{A g t}\rangle\rangle\left\langle\langle\langle\mathbb{A g t}\rangle\rangle \mathrm{N}\left(\mathrm{p}_{1} \wedge\right.\right.$ $\langle\langle\mathbb{A g t}\rangle\rangle\left\langle\mathrm{p}_{2}\right)$. That is, there is an $i R$-strategy $s_{\mathbb{A g t}}$ such that for all $\lambda \in$ out $t^{i_{s}}\left(q, s_{\mathbb{A g t}}\right)$ there is an $i \geq 0$ and an $i R$-strategy $s_{\mathbb{A g t}}^{\prime}$ such that for all $\lambda^{\prime} \in$ out $i_{s}\left(\lambda[i], s_{\mathbb{A g t}}^{\prime}\right)$ we have that $\lambda^{\prime}[0] \models \mathrm{p}_{1}$ and there is an $i R$-strategy $s_{\mathbb{A g t}}^{\prime \prime}$ such that for all $\lambda^{\prime \prime} \in$ out $t^{i_{s}}\left(\lambda^{\prime}[0], s_{\mathbb{A g t}}^{\prime \prime}\right)$ it holds that $\mathfrak{M}, \lambda^{\prime \prime} \models \diamond p_{2}$.

Because we have that $\left\{q^{\prime} \mid q^{\prime} \sim_{\mathbb{A g t}} \lambda[i]\right\}=\left\{q^{\prime} \mid q^{\prime} \sim_{\mathbb{A g t}} \lambda^{\prime}[0]\right\}$, we can take $s_{\mathbb{A g t}}^{\prime}$ as $s_{\mathbb{A g t}}^{\prime \prime}$. Then, we have that $\mathfrak{M}, q \models_{i_{S} R}\langle\langle\mathbb{A g t}\rangle\rangle \diamond\langle\langle\mathbb{A g t}\rangle\rangle \mathrm{N}\left(\mathrm{p}_{1} \wedge\langle\langle\mathbb{A g t}\rangle\rangle\left\langle\mathrm{p}_{2}\right)\right.$ iff there is an $i R$-strategy $s_{\mathbb{A g t}}$ such that for all $\lambda \in o u t^{i_{s}}\left(q, s_{\mathbb{A g t}}\right)$ there is an $i \geq 0$ and an $i R$-strategy $s_{\mathbb{A g t}}^{\prime}$ such that for all $\lambda^{\prime} \in$ out $i_{s}^{i_{s}}\left(\lambda[i], s_{\mathbb{A g t}}^{\prime}\right)$ we have that $\lambda^{\prime}[0] \models \mathrm{p}_{1}$ and $\mathfrak{M}, \lambda^{\prime} \models \diamond \mathrm{p}_{2}$.

Now, it is easy to see that we can combine $s_{\mathbb{A g t}}$ and each $s_{\mathbb{A g t}}^{\prime}$ to a single strategy $\hat{s}_{\mathbb{A g}}$ such that for all $\lambda \in \operatorname{out}^{i_{s}}\left(q, \hat{s}_{\text {Agt }}\right)$ it holds that $\mathfrak{M}, \lambda \models \Delta \mathrm{p}_{1} \wedge \Delta \mathrm{p}_{2}$. This shows that $\mathfrak{M}, q \models\left\langle\langle\mathbb{A g t}\rangle\left(\diamond \mathrm{p}_{1} \wedge \Delta \mathrm{p}_{2}\right)\right.$.

Apart from minor modifications, the next theorem, considering objective ability, is proven along the same lines.

Theorem 12 The sets $\operatorname{Val}\left(\boldsymbol{A T L _ { i _ { 0 } R } ^ { * }}\right)$ and $\operatorname{Val}\left(\boldsymbol{A T L}_{I r}^{*}\right)$ are incomparable, and similarly for $\boldsymbol{A T L}^{+}$.

Proof Again, we prove incomparability for $\mathbf{A T L}^{+}$. From this, incomparability for $\mathbf{A T L}^{*}$ follows immediately.

1. $\operatorname{Val}\left(\mathbf{A} \mathbf{T L} L_{I r}^{*}\right) \nsubseteq \operatorname{Val}\left(\mathbf{A} \mathbf{T L} \mathbf{i}_{i}^{*}\right)$. Suppose that $\operatorname{Val}\left(\mathbf{A} \mathbf{T L} L_{I r}^{*}\right) \subseteq \operatorname{Val}\left(\mathbf{A} \mathbf{T L}_{i_{0} R}^{*}\right)$. This implies that $\operatorname{Val}\left(\mathbf{A T L} \mathbf{L}_{I r}\right) \subseteq \operatorname{Val}\left(\mathbf{A} \mathbf{T L} i_{i_{0} R}\right)$ and by Theorem $4 \operatorname{Val}\left(\mathbf{A T L} \mathbf{L}_{I R}\right) \subseteq \operatorname{Val}\left(\mathbf{A} \mathbf{T L}_{i_{o} R}\right)$. But this contradicts Theorem 9.

2. $\operatorname{Val}\left(\mathbf{A} \mathbf{T L} \mathbf{i}_{i_{R}}^{*}\right) \nsubseteq \operatorname{Val}\left(\mathbf{A} \mathbf{T L} L_{I r}^{*}\right)$. For this case we consider the $\mathbf{A} \mathbf{T L}{ }^{*}$-formula

$$
\varphi=\langle\langle\mathbb{A g t}\rangle\rangle \diamond\left(\mathrm{p}_{1} \wedge\langle\langle\mathrm{Agt}\rangle\rangle\left\langle\mathrm{p}_{2}\right) \rightarrow\langle\langle\mathrm{Agt}\rangle\rangle\left(\diamond \mathrm{p}_{1} \wedge \diamond \mathrm{p}_{2}\right)\right.
$$

which is a validity of $\mathbf{A} \mathbf{T L}_{i_{o} R}^{*}$ but not of $\mathbf{A} \mathbf{T L}_{I r}^{*}$. The latter is shown by the same counterexample as used in the proof of Proposition 10 (we have $M_{5}, q_{0} \not \nvdash_{I r} \varphi$ ).

Finally, it remains to show that $\varphi \in \operatorname{Val}\left(\mathbf{A T L}_{i_{0} R}^{*}\right)$. This part is proven following the same idea as in the proof of Theorem 11 . We observe that every strategy $s_{\mathbb{A g}}$ of the grand coalition generates a unique path wrt. objective ability (because, in the objective semantics, possible paths starting from epistemic alternatives are not considered). This also means that uniformity of a strategy does not matter: there is no need to ever consider epistemic alternatives along a path. Hence, the two strategies witnessing $\langle\langle\mathbb{A g t}\rangle\rangle \diamond\left(\mathrm{p}_{1} \wedge\right.$ $\langle\langle\mathbb{A g t}\rangle\rangle\left\langle\mathrm{p}_{2}\right)$ can be combined to a single strategy witnessing $\langle\langle\mathbb{A g t}\rangle\rangle\left(\diamond \mathrm{p}_{1} \wedge \diamond \mathrm{p}_{2}\right)$.

5.6 Between subjective and objective ability for imperfect information $\left(i_{s}\right.$ vs. $\left.i_{o}\right)$

Finally, we compare validity sets for the semantic variants of ATL that differ on the outcome paths which are taken into account, i.e., whether only the paths representing the "objectively" possible courses of action are considered, or all the executions that are "subjectively" possible from the agents' perspective. 
Proposition 19 Formula $\Phi_{2} \equiv\langle\langle a\rangle\rangle \diamond \mathrm{p} \rightarrow \mathrm{p} \vee\langle\langle a\rangle\rangle \bigcirc\langle\langle a\rangle\rangle \diamond \mathrm{p}$ is valid in $\boldsymbol{A T L}_{i_{o} R}$ and $\boldsymbol{A T L}_{i_{o}}$,

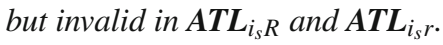

Proof We first prove validity of $\Phi_{2}$ in $\mathbf{A} \mathbf{T L}_{i_{o} r}$, which implies also validity in $\mathbf{A} \mathbf{T} \mathbf{L}_{i_{o} R}$ by Proposition 11. Suppose that $\mathfrak{M}, q \models_{i_{o} r}\langle\langle a\rangle\rangle \diamond \mathrm{p}$, then there must be an ir-strategy $s_{A}$ that enforces $\diamond p$ for every execution starting from $q$. But then, if $\mathrm{p}$ is not the case right at the beginning, $s_{A}$ must lead to a next state from which it enforces $\diamond \mathrm{p}$.

For the second part, invalidity of $\Phi_{2}$ in $\mathbf{A T L}_{i_{s} R}$ was already proved in Proposition 18. Thus, by Proposition 13, $\Phi_{2}$ is not valid in $\mathbf{A T L}_{i_{s} r}$, too.

In the next result we make use of the operator $\mathrm{N}$ introduced in Sect. 5.5.

\section{Proposition 20 Formula}

$$
\Phi_{8} \equiv\langle\langle a\rangle\rangle \mathrm{N}\langle\langle c\rangle\rangle \bigcirc\langle\langle a\rangle\rangle \mathrm{p} \rightarrow\langle\langle a, c\rangle\rangle \diamond \mathrm{p}
$$

is valid in $\boldsymbol{A T L}_{i_{s} R}$ and $\boldsymbol{A} \boldsymbol{T} \boldsymbol{L}_{i_{s} r}$, but invalid in $\boldsymbol{A} \boldsymbol{T} \boldsymbol{L}_{i_{o} R}$ and $\boldsymbol{A} \boldsymbol{T} \boldsymbol{L}_{i_{o} r}$.

Proof Analogously to Proposition 19, we prove the validity of $\Phi_{8}$ in $\mathbf{A} \mathbf{T} \mathbf{L}_{i_{s} r}$, and its invalidity in $\mathbf{A T L}_{i_{o} R}$.

First, let $\mathfrak{M}, q \models_{i_{s} r}\langle\langle a\rangle\rangle \mathrm{N}\langle\langle c\rangle\rangle \bigcirc\langle\langle a\rangle\rangle \bigcirc \mathrm{p}$. Then, for every state $q^{\prime} \in[q]_{\sim_{a}}, c$ has an irstrategy $s_{c}^{q^{\prime}}$ that enforces $\bigcirc\left\langle\langle a\rangle \bigcirc \mathrm{p}\right.$ from $\left[q^{\prime}\right] \sim_{c}$. By combining all these strategies into an $i r$-strategy $s_{c}$ (we can do it since the strategies $s_{c}^{q^{\prime}}$ are successful for whole indistinguishability classes of $c$ ), we have that $s_{c}$ enforces $\bigcirc\langle\langle a\rangle\rangle \bigcirc \mathrm{p}$ from every state in $[q]_{\sim_{\{a, c\}}}$, regardless of what the other players do (in particular, regardless of what $a$ does). But then, for every

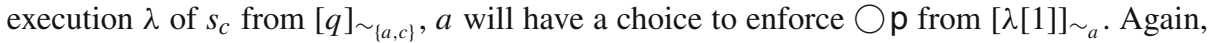
collecting these choices together yields an $i r$-strategy $s_{a}$ (we can fix the remaining choices arbitrarily). By taking $s_{\{a, c\}}=\left(s_{a}, s_{c}\right)$, we get a strategy for $\{a, c\}$ that enforces that $\mathrm{p}$ will be true in two steps, from every state in $[q]_{\sim_{\{a, c\}}}$. Hence, also $\mathfrak{M}, q \models_{i_{s} r}\langle\langle a, c\rangle\rangle \diamond \mathbf{p}$.

For the invalidity, consider the modified poor duck model $M_{2}$ from Fig. 13 augmented with additional agent $c$ that has no choice (i.e., at each state, it has only a single irrelevant action wait available). Let us denote the new iCGS by $M_{3}^{\prime}$, and let $\mathrm{p} \equiv$ shot. It is easy to see that $M_{2}^{\prime}, q_{0}^{\prime} \models_{i_{0} R}\langle\langle c\rangle\rangle \bigcirc\langle\langle a\rangle\rangle \bigcirc \mathrm{p}$, and hence also $M_{2}^{\prime}, q_{0}^{\prime} \models_{i_{0} R}\langle\langle a\rangle\rangle \mathrm{N}\langle\langle c\rangle\rangle \bigcirc\langle\langle a\rangle\rangle \bigcirc \mathrm{p}$. On the other hand, $M_{2}^{\prime}, q_{0}^{\prime} \nvdash_{i_{0} R}\langle\langle a, c\rangle\rangle \diamond \mathrm{p}$, which concludes the proof.

The following is an immediate consequence.

Theorem 13 For every $y, z \in\{R, r\}$, the sets $\operatorname{Val}\left(\boldsymbol{A T L} \boldsymbol{L}_{i_{s} y}\right)$ and $\operatorname{Val}\left(\boldsymbol{A T L} \boldsymbol{L}_{i_{o} z}\right)$ are incomparable, and similarly for $\boldsymbol{A T L}^{+}$and $\boldsymbol{A T L}^{*}$.

\section{Conclusions}

In this paper, we compare validity sets for different semantic variants of alternating-time temporal logic. In other words, we compare the general properties of games induced by different notions of ability. It is clear that changing the notions of strategy and success in a game leads to a different game. The issue considered here is whether, given a class of games, such a change leads to a different class of games, too. And, if so, what is the precise relationship between the two classes.

A summary of the results is presented in Fig. 10. The first and most important conclusion is that almost all the semantic variants discussed here are indeed different on the level of 
general properties they induce; before our study, it was by no means obvious. Moreover, our results show a very strong pattern: perfect information is a special case of imperfect information, perfect recall games are special case of memoryless games, and properties of objective and subjective abilities of agents are incomparable.

The relationships seem very natural, but they were surprisingly nontrivial to prove. This is best witnessed by Sect. 4 which comprises a third part of the paper only to construct appropriate tree-like unfoldings, and prove their equivalence to the original models. While embedding of perfect information in imperfect information is straightforward, the same cannot be said about embedding perfect recall in memoryless semantics—except when we disallow nested modalities. Consider e.g. the truth of formula $\langle\langle a\rangle\rangle \square\langle\langle a, b\rangle\rangle \diamond \mathrm{p}$ in a pointed iCGS $(\mathfrak{M}, q)$. Let $s_{a}$ be $a$ 's strategy that enforces $\langle\langle a, b\rangle\rangle \mathrm{p}$ to be always the case (suppose that such a strategy exists). After a history $h$, agent $a$ has different information when executing $s_{a}$ (because the agent has collected observations along $h$ from the root until now) than when we evaluate $\langle\langle a, b\rangle\rangle \diamond \mathrm{p}$ in the last state of $h$ (here, the collecting of observations starts anew). In consequence, the "straightforward" unfolding of $(\mathfrak{M}, q)$ endows agents with too much information when nested strategic formulas are evaluated, and the correctness of the construction is not automatic. For objective abilities, we prove that the standard unfolding still works because path formulae of ATL* (that can be seen as "winning conditions" in the corresponding game) do not discern between the two epistemic positions. For subjective abilities, the unfolding does not work, but it can be recovered by a technical construction with "hanging" subtrees added to the basic tree. This construction is among the main contributions of this paper.

Technical subtleties aside, the most interesting contribution lies possibly in our noninclusion results. First, they show that the language of ATL is sufficiently expressive to distinguish between the main notions of ability. Moreover, non-inclusion is demonstrated on formulae encoding intuitive and well known properties, like fixpoint characterizations of strategic/temporal modalities and the duality between necessary and obtainable outcomes. It is important to see in which semantics the formulae hold, and in which they do not hold. Finally, although the proofs of non-inclusion are very comprehensive (since they are based on counterexamples), finding the counterexamples required expertise and was not straightforward either.

Another interesting outcome of the study is that the type of information has strictly more impact on validities than the type of recall in the language of ATL but not in $\boldsymbol{A T L}^{*}$. In particular the validity sets of $\mathbf{A} \mathbf{T L}_{I r}^{*}$ and $\mathbf{A T L}_{i R}^{*}$ are incomparable. This suggests that $\mathbf{A T L} \mathbf{T}^{*}$ allows to specify significantly subtler properties of strategic play than the more restricted language of ATL.

Acknowledgments The authors would like to express their thanks to Jan Broersen for the argument that triggered this study, Valentin Goranko for discussions on (non-) validity of fixpoint properties in various semantics of ATL, and anonymous reviewers of EUMAS'10, IJCAI'11 and JAAMAS for their valuable remarks. Wojciech Jamroga acknowledges the support of the FNR (National Research Fund) Luxembourg under projects S-GAMES - C08/IS/03 and GALOT_INTER/DFG/12/06.

\section{Appendix: Proofs}

\subsection{Proofs of Sect. 4.2}

The following Lemma is obvious by the definition of $i_{o} R$-tree unfoldings. It states that that nodes group indistinguishable in the tree unfolding are also group indistinguishable in the model if interpreted as histories. 
Lemma 1 Let $\mathfrak{M}$ be an $i$ CGS, $h_{1}$ and $h_{2}$ two nodes in its $i_{o} R$-tree unfolding, and $A \subseteq \mathbb{A g t}$ a group of agents. If $h_{1} \sim_{A}^{T_{o}(\mathfrak{M}, q)} h_{2}$ then $h_{1} \approx_{A}^{\mathfrak{M}} h_{2}$.

Moreover, we have that all histories indistinguishable in the model are also indistinguishable in the tree if only states reachable from the current state are considered.

Lemma 2 Let $\mathfrak{M}$ be an $i$ CGS, $A \subseteq \mathbb{A g t}$ and $h$ a node in $T_{o}(\mathfrak{M}, q)$. Then, for all $h_{1}, h_{2} \in$ $\Lambda_{\mathfrak{M}}($ last $(h))$ we have that

$$
\text { if } h_{1} \approx_{A}^{\mathfrak{M}} h_{2} \text { then } h\left(h_{1}[1, \infty]\right) \sim_{A}^{T_{o}(\mathfrak{M}, q)} h\left(h_{2}[1, \infty]\right) .
$$

Theorem 2 For every $\boldsymbol{i C G S} \mathfrak{M}$, state $q$ in $\mathfrak{M}$, and $\boldsymbol{A T L}{ }^{*}$-formula $\varphi$ we have:

$$
\mathfrak{M}, q \models_{i_{o} R} \varphi \text { iff } T_{o}(\mathfrak{M}, q), q \models_{i_{o} R} \varphi \text { iff } T_{o}(\mathfrak{M}, q), q \models_{i_{o} r} \varphi .
$$

Proof We show that, for every node $h$ in $T_{o}(\mathfrak{M}, q)$, it holds that $\mathfrak{M}$, last $(h) \models_{i_{o} R} \varphi$ iff $T_{o}(\mathfrak{M}, q), h \models_{i_{o} r} \varphi$. Then, the claim follows by Propositions 4 and 5 and for $h=q$. The proof is done by induction on the structure of $\varphi$.

Base cases:

Propositional case: Straightforward.

Case: $\varphi \equiv\langle\langle A\rangle\rangle \gamma$ where $\gamma$ contains no nested strategic modalities.

“ $\Rightarrow$ ": Suppose that $\mathfrak{M}$, last $(h) \models_{i_{o} R}\langle\langle A\rangle\rangle \gamma$. So, there is an $i_{o} R$-strategy $s_{A}$ such that

$$
(\star) \forall \lambda \in \operatorname{out}_{\mathfrak{M}}\left(\operatorname{last}(h), s_{A}\right): \mathfrak{M}, \lambda \models_{i_{o} R} \gamma .
$$

We construct the memoryless strategy $s_{A}^{\prime}$ in $T_{o}(\mathfrak{M}, q)$ as follows: $s_{a}^{\prime}\left(\hat{h} h^{\prime}\right)=s_{a}\left(\right.$ last $\left.(h) h^{\prime}\right)$ for every $a \in A$ and $\hat{h}$ such that $h \sim_{A}^{T_{o}(\mathfrak{M}, q)} \hat{h}$. For all other histories $h^{\prime \prime}$ (which do not have the form $\left.\hat{h} h^{\prime}\right)$ we define $s_{a}^{\prime}\left(h^{\prime \prime}\right)$ arbitrarily but in a uniform way. It is easy to see that $s_{A}^{\prime}$ is uniform: For two histories $h_{1}=\hat{h}^{\prime} h^{\prime}$ and $h_{2}=\hat{h}^{\prime \prime} h^{\prime \prime}$ with $\hat{h}^{\prime} \sim_{A}^{T_{o}(\mathfrak{M}, q)} h$ and $\hat{h}^{\prime \prime} \sim_{A}^{T_{o}(\mathfrak{M}, q)} h$ and $h_{1} \sim_{A}^{T_{o}(\mathfrak{M}, q)} h_{2}$ we have $s_{A}^{\prime}\left(h_{1}\right)=s_{A}^{\prime}\left(h_{2}\right)$; for, $h_{1} \sim_{A}^{T_{o}(\mathfrak{M}, q)} h_{2}$ implies $h_{1} \approx_{A}^{\mathfrak{M}} h_{2}$ (by Lemma 1 ) and thus $s_{A}\left(\right.$ last $\left.(h) h^{\prime}\right)=s_{A}\left(\right.$ last $\left.(h) h^{\prime \prime}\right)$.

By construction of $s_{A}^{\prime}$ we have that

$$
\text { last }(h) q_{1} q_{2} \cdots \in \operatorname{out}_{\mathfrak{M}}\left(\operatorname{last}(h), s_{A}\right) \text { iff }(h)\left(h q_{1}\right)\left(h q_{1} q_{2}\right) \cdots \in \operatorname{out}_{T_{o}(\mathfrak{M}, q)}\left(h, s_{A}^{\prime}\right) .
$$

Since the valuation of propositions does only depend on the final state of a history and by $(\star)$ we have $T_{o}(\mathfrak{M}, q), h \models i_{i_{o}}\langle\langle A\rangle\rangle \gamma$.

$\Leftarrow$ : Suppose we have $T_{o}(\mathfrak{M}, q), h \models_{i_{o} r}\langle\langle A\rangle\rangle$. So there is an $i_{o} r$-strategy $s_{A}$ such that

$$
(\star) \forall \lambda \in \operatorname{out}_{T_{o}(\mathfrak{M}, q)}\left(h, s_{A}\right): T_{o}(\mathfrak{M}, q), \lambda \models i_{o} r \gamma .
$$

We construct a witnessing $i_{o} R$-strategy $s_{A}^{\prime}$ in $\mathfrak{M}$ as follows: $s_{a}^{\prime}(\hat{h})=s_{a}\left(h h^{\prime}\right)$ for every $a \in A$ and $\hat{h}$ such that last $(h) h^{\prime} \approx_{a}^{\mathfrak{M}} \hat{h}$ and last $(h) h^{\prime} \in \Lambda_{\mathfrak{M}}^{\text {fin }}(\operatorname{last}(h))$. We define $s_{a}^{\prime}$ arbitrarily for all other histories with the condition to assign the same actions to indistinguishable histories in $\mathfrak{M}$. The definition of $s_{a}^{\prime}$ does only take into account the subtree starting at $h$. Then, by Lemma 2 we have that strategy $s_{A}^{\prime}$ is uniform by construction. Note, that it may differ from $s_{A}$ but only for histories which are not realizable given that the initial state is last $(h)$.

By construction of $s_{A}^{\prime}$, we also have

$$
(h)\left(h q_{1}\right)\left(h q_{1} q_{2}\right) \cdots \in \operatorname{out}_{T_{o}(\mathfrak{M}, q)}\left(h, s_{A}\right) \text { iff last }(h) q_{1} q_{2} \cdots \in \operatorname{out}_{\mathfrak{M}}\left(\operatorname{last}(h), s_{A}^{\prime}\right) \text {. }
$$

Since the valuation of propositions does only depend on the final state of a history and by $(\star)$ we have $\mathfrak{M}$, last $(h) \models_{i_{o} R}\langle\langle A\rangle \gamma$. 
Fig. 15 General setting of the proof of Proposition 7

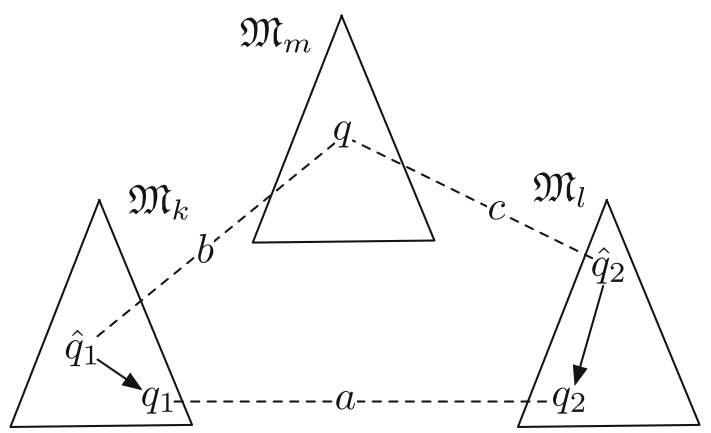

Induction step:

Case: $\varphi \equiv \psi_{1} \wedge \psi_{2}$. Straightforward.

Case: $\varphi \equiv \neg \psi$. M, last $(h) \models_{i_{o} R} \neg \psi$ iff not $\mathfrak{M}$, last $(h) \models_{i_{o} R} \psi$ iff (by induction hypothesis) not $T_{o}(\mathfrak{M}, q), h \models_{i_{o} R} \psi$ iff $T_{o}(\mathfrak{M}, q), h \models_{i_{o} R} \neg \psi$.

Case: $\varphi \equiv\left\langle\langle A\rangle \gamma\right.$. By induction hypothesis we have for each history $h$ in $T_{o}(\mathfrak{M}, q)$ and each strict state-subformula $\varphi^{\prime}$ of $\gamma$ that $\mathfrak{M}$, last $(h) \models_{i_{o} R} \varphi^{\prime}$ iff $T_{o}(\mathfrak{M}, q), h \models_{i_{o} r} \varphi^{\prime}$. For any maximal strict subformula $\varphi^{\prime}$ of $\varphi$ we label all states $h$ in $T_{o}(\mathfrak{M}, q)$ and states last $(h)$ in $\mathfrak{M}$ with a new proposition $\mathrm{p}_{\varphi^{\prime}}$ iff $\varphi^{\prime}$ holds in this very state. Then, we replace each $\varphi^{\prime}$ in $\varphi$ with proposition $\mathbf{p}_{\varphi^{\prime}}$. This yields a formula without nested modalities and the claim follows by induction.

\subsection{Proofs of Sect. 4.3}

In this section we give all details needed to prove Theorem 3. The structure of the proof is shown in Fig. 9.

The following lemma is essential to show that truth in $i_{S} R$-pando-like models is insensitive to the type of available strategies (memoryless vs. perfect recall). The lemma is needed to show that a uniform perfect recall strategy in the pando-like model gives rise to a uniform memoryless strategy. Therefore, we have to show that two states which are indistinguishable in the model give rise to indistinguishable histories.

Lemma 3 Let $\mathfrak{M}$ be an $i_{s} R$-pando like $i$ CGS and $q, q_{1}, \hat{q}_{1}, q_{2}, \hat{q}_{2} \in$ St where $q_{i}$ is reachable from $\hat{q}_{i}$, i.e. $\rho\left(\hat{q}_{i}, q_{i}\right) \neq \epsilon$, for $i=1$, 2. Moreover, let $\hat{q}_{1} \sim_{b}^{\mathfrak{M}} q \sim_{c}^{\mathfrak{M}} \hat{q}_{2}$ for some $b, c \in A$ and $q_{1} \sim \underset{a}{\mathfrak{M}} q_{2}$. Then, we have that $\rho\left(\hat{q}_{1}, q_{1}\right) \approx_{a}^{\mathfrak{M}} \rho\left(\hat{q}_{2}, q_{2}\right)$.

Proof The setting is illustrated in Fig. 15. In the following we consider all possibilities how $q, \hat{q}_{1}, \hat{q}_{2}, q_{1}$, and $q_{2}$ can be located. We recall that $\rho_{\mathfrak{M}_{k}}\left(q^{\prime}\right)=q^{\prime}$ means that $q^{\prime}$ is the root node of model $\mathfrak{M}_{k}$. We assume that $k, l, m \in I$ where $I$ is the index set from Definition 16.

Case 1: $q_{1} \hat{\sim}_{a} q_{2}$. Let $q_{1} \in \mathfrak{M}_{k}$ and $q_{2} \in \mathfrak{M}_{l}, k \neq l$.

Case 1.1: $\rho_{\mathfrak{M}_{k}}\left(q_{1}\right)=q_{1}$. That is, $q_{1}$ is the root node of $\mathfrak{M}_{k}$. We have $\hat{q}_{1}=q_{1} \sim \stackrel{\mathfrak{M}}{b}$. Then, by Definition $16.5\left|\rho\left(\hat{q}_{2}\right)\right|=\left|\rho\left(q_{2}\right)\right|$ which implies $\hat{q}_{2}=q_{2}$. Hence, we have $\rho_{\mathfrak{M}_{k}}\left(\hat{q}_{1}, q_{1}\right)=q_{1} \approx_{a}^{\mathfrak{M}} \rho_{\mathfrak{M}_{l}}\left(\hat{q}_{2}, q_{2}\right)=q_{2}$ and are done.

Case $1.2 \rho_{\mathfrak{M}_{k}}\left(q_{2}\right)=q_{2}$. Analogously to Case 1.1.

Case $1.3 \rho_{\mathfrak{M}_{k}}\left(q_{1}\right) \hat{\approx}_{a}^{\mathfrak{M}} \rho_{\mathfrak{M}_{l}}\left(q_{2}\right)$ and both $q_{1}$ and $q_{2}$ are not root nodes.

Case 1.3.1 $\hat{q}_{1} \hat{\sim}_{b}^{\mathfrak{M}} q$. Let $q \in S t_{m}$ with $m \neq k$. 
Case 1.3.1.1 $\rho_{\mathfrak{M}_{k}}\left(\hat{q}_{1}\right)=\hat{q}_{1}$. Then, $\hat{q}_{1} \hat{\sim}_{a}^{\mathfrak{M}} q^{\prime}$ where $q^{\prime} \in S t_{l}$ is the root node of $\mathfrak{M}_{l}$. But then, by Definition 16.5 we have $\left|\rho\left(q^{\prime}\right)\right|=\left|\rho\left(\hat{q}_{2}\right)\right|$ and thus $\rho_{\mathfrak{M}_{l}}\left(\hat{q}_{2}\right)=\hat{q}_{2}$. This proves that $\rho_{\mathfrak{M}_{k}}\left(\hat{q}_{1}, q_{1}\right) \approx_{a}^{\mathfrak{M}} \rho_{\mathfrak{M}_{l}}\left(\hat{q}_{2}, q_{2}\right)$.

Case 1.3.1.2 $\rho_{\mathfrak{M}_{m}}(q)=q$. Again, by Definition 16.5 following the same reasoning as in Case 1.3.1.1 we obtain $\rho_{\mathfrak{M}_{l}}\left(\hat{q}_{2}\right)=\hat{q}_{2}$. Showing that $\rho_{\mathfrak{M}_{k}}\left(\hat{q}_{1}, q_{1}\right) \approx_{a}^{\mathfrak{M}} \rho_{\mathfrak{M}_{l}}\left(\hat{q}_{2}, q_{2}\right)$.

Case 1.3.1.3 $\rho_{\mathfrak{M}_{k}}\left(\hat{q}_{1}\right) \hat{\approx}_{a}^{\mathfrak{M}} \rho_{\mathfrak{M}_{m}}(q)$ and neither $\hat{q}_{1}$ nor $q$ are root nodes. Then, we either have that $l=m$ and $\rho_{\mathfrak{M}_{l}}\left(\hat{q}_{2}\right) \approx{ }_{a}^{\mathfrak{M}} \rho_{\mathfrak{M}_{l}}(q)$ which implies that $\rho_{\mathfrak{M}_{k}}\left(\hat{q}_{1}, q_{1}\right) \hat{\approx}_{a}^{\mathfrak{M}} \rho_{\mathfrak{M}_{l}}\left(\hat{q}_{2}, q_{2}\right)$. Or, $l \neq m$ and we have to distinguish again two cases. If $\hat{q}_{2}$ is the root node; then, it is connected with the root $q^{\prime} \in S t_{k}$ of $\mathfrak{M}_{k}$ by $\sim \mathfrak{a}$. We have $q^{\prime} \sim \underset{a}{\mathfrak{M}} \hat{q}_{2} \sim \underset{c}{\mathfrak{M}} q \sim_{b}^{\mathfrak{M}} \hat{q}_{1}$ and by Definition 16.5 it must be the case that $\left|\rho\left(q^{\prime}\right)\right|=\left|\rho\left(\hat{q}_{1}\right)\right|$. Contradiction. Hence, we can safely assume that $\hat{q}_{2}$ is not a root. Then, $\rho_{\mathfrak{M}_{l}}\left(\hat{q}_{2}\right) \approx_{c}^{\mathfrak{M}}$ $\rho_{\mathfrak{M}_{m}}(q) \approx_{b}^{\mathfrak{M}} \rho_{\mathfrak{M}_{k}}\left(\hat{q}_{1}\right)$. So, all these states are on the same height level which implies that $\rho_{\mathfrak{M}_{k}}\left(\hat{q}_{1}, q_{1}\right) \approx_{a}^{\mathfrak{M}} \rho_{\mathfrak{M}_{l}}\left(\hat{q}_{2}, q_{2}\right)$.

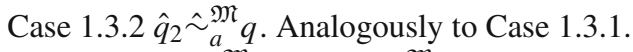

Case 1.3.3 $\hat{q}_{1} \sim \sim_{a}^{\mathfrak{M}_{k}} q$ or $\hat{q}_{2} \sim \sim_{a}^{\mathfrak{M}_{l}} q$. In each of these cases it means that either $q \notin S t_{k}$ or $q \notin S t_{l}$ as $k \neq l$. Case 1.3.1 or Case 1.3.2 applies.

Case 2: $q_{1} \sim_{a}^{\mathfrak{M}_{k}} q_{2}$. Then, by definition $k=l$ and $\rho_{\mathfrak{M}_{k}}\left(q_{1}\right) \approx_{a}^{\mathfrak{M}} \rho_{\mathfrak{M}_{k}}\left(q_{2}\right)$.

Case 2.1: $q \in S t_{k}$. We have $\left|\rho_{\mathfrak{M}_{k}}\left(\hat{q}_{1}\right)\right|=\left|\rho_{\mathfrak{M}_{k}}(q)\right|=\left|\rho_{\mathfrak{M}_{k}}\left(\hat{q}_{2}\right)\right|$ which follows from the assumption $\hat{q}_{1} \sim_{b}^{\mathfrak{M}_{k}} q \sim_{c}^{\mathfrak{M}_{k}} \hat{q}_{2}$; hence also, $\rho_{\mathfrak{M}_{k}}\left(\hat{q}_{1}, q_{1}\right) \hat{\approx}_{a}^{\mathfrak{M}} \rho_{\mathfrak{M}_{k}}\left(\hat{q}_{2}, q_{2}\right)$.

Case 2.2: $q \in S t_{m}, m \neq k$. Then, we have $\hat{q}_{1} \hat{\sim}_{b}^{\mathfrak{M}} q \hat{\sim}_{c}^{\mathfrak{M}} \hat{q}_{2}$. Again we have to distinguish the different cases how $q$ is connected to $\hat{q}_{1}$ and $\hat{q}_{2}$ respectively.

Case 2.1.1 $\rho_{\mathfrak{M}_{m}}(q)=q$. That is, we assume that $q$ is a root node. By Definition 16.5 we have $\left|\rho\left(\hat{q}_{1}\right)\right|=\left|\rho\left(\hat{q}_{2}\right)\right|$ and $\rho\left(\hat{q}_{1}, q_{1}\right) \approx_{a}^{\mathfrak{M}} \rho\left(\hat{q}_{2}, q_{2}\right)$ follows.

Case 2.1.2 $\rho_{\mathfrak{M}_{m}}(q) \neq q$. We have $\rho_{\mathfrak{M}_{k}}\left(\hat{q}_{1}\right) \hat{\approx}_{b}^{\mathfrak{M}} \rho_{\mathfrak{M}_{l}}(q)$ and $\rho_{\mathfrak{M}_{k}}\left(\hat{q}_{2}\right) \hat{\approx}_{c}^{\mathfrak{M}} \rho_{\mathfrak{M}_{l}}(q)$ which implies $\left|\rho_{\mathfrak{M}_{k}}\left(\hat{q}_{1}\right)\right|=\left|\rho_{\mathfrak{M}_{m}}(q)\right|=\left|\rho_{\mathfrak{M}_{k}}\left(\hat{q}_{2}\right)\right|$ and hence $\rho_{\mathfrak{M}_{k}}\left(\hat{q}_{1}, q_{1}\right) \approx_{a}^{\mathfrak{M}}$ $\rho_{\mathfrak{M}_{k}}\left(\hat{q}_{2}, q_{2}\right)$.

The next lemma analyses the structure of two indistinguishable nodes from subsequent tree levels.

Lemma 4 Let $\mathfrak{M}$ be an iCGS, $q$ a state in it, $h_{1} \in \Delta_{\mathfrak{M}}^{i}(q), h_{2} \in \Delta_{\mathfrak{M}}^{i+1}(q)$, and $h_{1} \sim_{a}^{T_{s}}(\mathfrak{M}, q)$ $h_{2}$ for some $i \in \mathbb{N}_{0}$. Then, we have that lastr $\left(h_{1}\right) \underset{a}{\sim} \underset{\mathfrak{M}}{\operatorname{rel}}\left(h_{2}\right)$.

Proof By definition, we have that $\operatorname{ref}\left(h_{2}\right) \sim_{a}^{T_{s}(\mathfrak{M}, q)} h_{1}$ and lastr $\left(\operatorname{ref}\left(h_{2}\right)\right) \sim_{a}^{\mathfrak{M}} \operatorname{rel}\left(h_{2}\right)$. Because $\operatorname{ref}\left(h_{2}\right) \in \Delta_{\mathfrak{M}}^{i}(q)$ we also have $\operatorname{rel}\left(h_{1}\right) \approx_{a}^{\mathfrak{M}} \operatorname{rel}\left(\operatorname{ref}\left(h_{2}\right)\right)$, and hence $\operatorname{lastr}\left(h_{1}\right) \sim \mathfrak{M}$ $\operatorname{lastr}\left(\operatorname{ref}\left(h_{2}\right)\right)$. The claim follows because lastr $\left(\operatorname{ref}\left(h_{2}\right)\right) \sim \underset{a}{\mathfrak{M}} \operatorname{rel}\left(h_{2}\right)$ and by the transitivity of $\underset{a}{\sim}$.

The next lemma states that nodes which are indistinguishable for a group of agents must be located on subsequent or the same level of the pando unfolding; moreover, it characterizes the structure of these nodes.

Lemma 5 Let $\mathfrak{M}$ be an $i$ CGS, $q$ a state in it, and $A \subseteq \mathbb{A g t}$ be a group of agents. Then, for all $h \in S t_{T_{s}(\mathfrak{M}, q)}$ there is an $i \in \mathbb{N}_{0}$ such that for all $h^{\prime} \in S t_{T_{s}(\mathfrak{M}, q)}$ with $h\left(\sim_{A}^{T_{s}(\mathfrak{M}, q)}\right)^{*} h^{\prime}$ we have that $h, h^{\prime} \in \Delta_{\mathfrak{M}}^{i}(q) \cup \Delta_{\mathfrak{M}}^{i+1}(q)$; moreover, if $h^{\prime} \in \Delta_{\mathfrak{M}}^{i+1}(q) h\left(\sim_{A}^{T_{s}(\mathfrak{M}, q)}\right)^{*} h^{\prime}$ and there is an $h^{\prime \prime} \in \Delta_{\mathfrak{M}}^{i}(q)$ with $h\left(\sim_{A}^{T_{s}(\mathfrak{M}, q)}\right)^{*} h^{\prime \prime}$ then $\operatorname{rel}\left(h^{\prime}\right) \in \operatorname{St} t_{\mathfrak{M}}$ and jump $\left(h^{\prime}\right) \in A$. 
Proof We write $T_{s}$ for $T_{S}(\mathfrak{M}, q)$ and $\Delta^{i}$ for $\Delta_{\mathfrak{M}}^{i}(q)$ and so on. We proceed by induction on the length of the epistemic path $h^{\prime}=h_{1} \sim_{a_{1}} \cdots \sim_{a_{l+1}} h_{l+1}$. We show the following: (i) if $h_{j} \in \Delta^{i}$ for all $j=1, \ldots, l$ then $h_{l+1} \in \Delta^{i-1} \cup \Delta^{i} \cup \Delta^{i+1}$; if this is not the case then (ii) if $h_{j} \in \Delta^{i} \cup \Delta^{i+1}$ for all $j=1, \ldots, l$ then $h_{l+1} \in \Delta^{i} \cup \Delta^{i+1}$ and for each $h_{j} \in \Delta^{i+1}$ we have that $\operatorname{rel}\left(h_{j}\right) \in \operatorname{St}, \operatorname{jump}\left(h_{j}\right) \in A$, and $\operatorname{ref}\left(h_{j}\right) \in \Delta^{i}$.

Base case The case for $h \sim_{a} h^{\prime}$ is clear by definition.

Induction step Suppose we have $h^{\prime}=h_{1} \sim_{a_{1}} \cdots \sim_{a_{l}} h_{l}$ satisfying the assumption and assume that $h_{l} \sim_{a_{l+1}} h_{l+1}$. Firstly, assume that case (i) applies; that is, that all $h_{j} \in \Delta^{i}$ for $j=1, \ldots, l$. By definition $h_{l+1} \in \Delta^{i-1} \cup \Delta^{i} \cup \Delta^{i+1}$. Moreover, if $h_{l+1} \in \Delta^{i+1}$ then it must have the required form $h^{\prime \prime} \hat{a} q^{\prime}$ by Definition 17(c).

We consider case (ii). Firstly, suppose that $h_{l} \in \Delta^{i+1}$ and $h_{l}=h^{\prime} \hat{a}_{l} q^{\prime}$. We consider $h_{l} \sim_{a_{l+1}} h_{l+1}$. By definition $h_{l+1} \in \Delta^{i} \cup \Delta^{i+1} \cup \Delta^{i+2}$. If $h_{l+1} \in \Delta^{i+1}$ it also has the required form. For the sake of contradiction, suppose that $h_{l+1} \in \Delta^{i+2}$. Then, $h_{l+1}=h^{\prime \prime} \hat{a}_{l+1} q^{\prime \prime}$ for some $h^{\prime \prime} \in \Delta^{i+1}$ with $h^{\prime \prime} \sim_{a_{l+1}} h_{l}$. In this case, $h^{\prime \prime}$ does also have the form $h^{\prime \prime}=h^{\prime \prime \prime} \hat{a}_{l} q^{\prime \prime \prime}$ and therewith $h_{l+1}=h^{\prime \prime \prime} \hat{a}_{l} q^{\prime \prime \prime} \hat{a}_{l+1} q^{\prime \prime}$ contradicting $h_{l+1} \in \Delta^{i+2}$ by definition of the sets $\Delta^{j}$.

Secondly, if $h_{l} \in \Delta^{i}$ we have that $h_{l+1} \in \Delta^{i-1} \Delta^{i} \cup \Delta^{i+1}$ and that $h_{l+1}$ has the required form if $h_{l+1} \in \Delta^{i+1}$ following the very same reasoning as in case (i). Moreover, it cannot be the case that $h_{l+1} \in \Delta^{i-1}$. To see this, we observe that there is some $h_{u}$ with $1 \leq u<l+1$, $h_{u} \in \Delta^{i+1}$ and $h_{l+1}\left(\sim_{A}^{T_{s}}\right)^{*} h_{u}$. Now the reasoning of the previous case can be applied to obtain a contradiction.

The next lemma states that the relevant parts (i.e. histories) of two states of the pando unfolding are group indistinguishable in the model if the states are group indistinguishable in the pando unfolding and are located within the same tree (i.e. share the same root node).

Lemma 6 Let $h_{1}\left(\sim_{A}^{T_{s}}\right)^{*} h_{2}$. If there is an $i \in \mathbb{N}_{0}$ with $h_{1}, h_{2} \in \Delta^{i}$ then $\operatorname{rel}\left(h_{1}\right)\left(\approx_{A}^{\mathfrak{M}}\right)^{*} \operatorname{rel}\left(h_{2}\right)$.

Proof The poof is done by induction on the number of epistemic steps between $h_{1}$ and $h_{2}$. More precisely, we show that for all $h^{\prime}$ with $h_{1}\left(\sim_{A}^{T_{S}}\right)^{*} h^{\prime}$ we have that

(i) $\operatorname{rel}\left(h_{1}\right)\left(\approx_{A}^{\mathfrak{M}}\right)^{*} \operatorname{rel}\left(h^{\prime}\right)$ if $h^{\prime} \in \Delta^{i}$;

(ii) $\operatorname{ref}\left(h^{\prime}\right)\left(\sim_{A}^{T_{S}}\right)^{*} h_{1}$ and $\operatorname{lastr}\left(h_{1}\right)\left(\sim_{A}^{\mathfrak{M}}\right)^{*} \operatorname{lastr}\left(\operatorname{ref}\left(h^{\prime}\right)\right)$ if $h^{\prime} \in \Delta^{i+1}$;

(iii) and $\operatorname{rel}\left(h_{1}\right)\left(\sim_{A}^{\mathfrak{M}}\right)^{*} \operatorname{lastr}\left(h^{\prime}\right)$ if $h^{\prime} \in \Delta^{i-1}$ (and $i>0$ ).

The base cases are clear by definition. We assume that $h_{1}\left(\sim_{A}^{T_{S}}\right)^{*} h^{\prime}, i>0$ and we show that $h_{2}$ with $h_{1}\left(\sim_{A}^{T_{s}}\right)^{*} h^{\prime} \sim_{a}^{T_{s}} h_{2}$ for $a \in A$ satisfies the property of the lemma.

Case: $h^{\prime} \in \Delta^{i}$ and $h_{2} \in \Delta^{i}$. By definition $\operatorname{rel}\left(h^{\prime}\right) \approx_{a}^{\mathfrak{M}} \operatorname{rel}\left(h_{2}\right)$ and by induction $\operatorname{rel}\left(h_{1}\right)\left(\approx_{A}^{\mathfrak{M}}\right) * \operatorname{rel}\left(h^{\prime}\right)$; hence, $\operatorname{rel}\left(h_{1}\right)\left(\approx_{A}^{\mathfrak{M}}\right) * \operatorname{rel}\left(h_{2}\right)$.

Case: $h^{\prime} \in \Delta^{i}$ and $h_{2} \in \Delta^{i+1}$. By induction, $\operatorname{rel}\left(h_{1}\right)\left(\approx_{A}^{\mathfrak{M}}\right)^{*} \operatorname{rel}\left(h^{\prime}\right)$ and in particular, lastr $\left(h_{1}\right) \sim_{A}^{*} \operatorname{lastr}\left(h^{\prime}\right)$. By Lemma $4 \operatorname{lastr}\left(h^{\prime}\right) \sim_{a}^{\mathfrak{M}} \operatorname{rel}\left(h_{2}\right)$. This shows that, $\operatorname{lastr}\left(h_{1}\right)\left(\sim_{A}^{\mathfrak{M}}\right) * \operatorname{rel}\left(h_{2}\right) \sim \underset{a}{\mathfrak{M}} \operatorname{lastr}\left(\operatorname{ref}\left(h_{2}\right)\right)$.

Case: $h^{\prime} \in \Delta^{i}$ and $h_{2} \in \Delta^{i-1}$. By definition $h_{2} \sim_{A}^{T_{S}} \operatorname{ref}\left(h^{\prime}\right)$ and $\operatorname{rel}\left(h^{\prime}\right) \sim_{A}^{\mathfrak{M}} \operatorname{lastr}\left(h_{2}\right)$; hence, $\operatorname{rel}\left(h^{\prime}\right) \in S t_{\mathfrak{M}}$. Thus, by induction $\operatorname{rel}\left(h_{1}\right)\left(\sim_{A}^{\mathfrak{M}}\right)^{*} \operatorname{rel}\left(h^{\prime}\right)$ and by Lemma 4 $\operatorname{rel}\left(h^{\prime}\right) \sim \underset{a}{\mathfrak{M}} \operatorname{lastr}\left(h_{2}\right)$. This shows that $\operatorname{rel}\left(h_{1}\right)\left(\sim_{A}^{\mathfrak{M}}\right) * \operatorname{lastr}\left(h_{2}\right)$.

Case: $h^{\prime} \in \Delta^{i-1}$ and $h_{2} \in \Delta^{i-1}$. Follows immediately.

Case: $h^{\prime} \in \Delta^{i-1}$ and $h_{2} \in \Delta^{i}$. We have $\operatorname{rel}\left(h_{1}\right)(\sim \underset{A}{\mathfrak{M}})^{*} \operatorname{lastr}\left(h^{\prime}\right)$. By Lemma $4 \operatorname{rel}\left(h_{2}\right) \sim_{a}^{\mathfrak{M}}$ $\operatorname{lastr}\left(h^{\prime}\right)$ and hence $\operatorname{rel}\left(h_{1}\right)\left(\sim_{A}^{\mathfrak{M}}\right)^{*} \operatorname{rel}\left(h_{2}\right)$. The claim follows as $\operatorname{rel}\left(h_{1}\right), \operatorname{rel}\left(h_{2}\right) \in S t_{\mathfrak{M}}$.

Cases where $h^{\prime} \in \Delta^{j}$ and $h_{2} \in \Delta^{k}$ with $|j-k|>1$ are not possible due to Lemma 5 . 
Lemma 7 For all $q$ in $\mathfrak{M}$ and all $i, j \in \mathbb{N}_{0}$ with $i \neq j$ we have that $\Delta_{\mathfrak{M}}^{i}(q) \cap \Delta_{\mathfrak{M}}^{j}(q)=\emptyset$.

Lemma 8 Let $\mathfrak{M}$ be an $i$ CGS, $q$ a state in it, and $a \in \mathbb{A g t}$. Every relation $\sim_{a}^{T_{s}(\mathfrak{M}, q)}$ is an equivalence relation.

Proof We write $T_{s}$ for $T_{S}(\mathfrak{M}, q)$. Reflexivity and symmetry of epistemic relations in $T_{S}$ are clear from the definition of $i_{s} R$-pando unfoldings, but we need to prove transitivity. Suppose that $h_{1} \sim_{a}^{T_{s}} h_{2}$ and $h_{2} \sim \sim_{a}^{T_{s}} h_{3}$. We have to show that $h_{1} \sim_{a}^{T_{s}} h_{3}$. The proof is done by induction on the level $\Delta^{i}$. The base case for $\Delta^{0}$ is clear from the transitivity of the standard indistinguishability relation $\approx_{a}^{\mathfrak{M}}$. By Lemma 5 (and the symmetry of $\sim^{T_{s}}$ ) it is sufficient to consider the following cases (we assume that $i>0$ ):

$h_{1}, h_{2}, h_{3} \in \Delta^{i}:$ Follows by the transitivity of $\approx_{a}^{\mathfrak{M}}$ (induction hypothesis).

$h_{1}, h_{2} \in \Delta^{i}, h_{3} \in \Delta^{i+1}:$ From $h_{1} \sim_{a}^{T_{s}} h_{2}$ it follows that $\operatorname{rel}\left(h_{1}\right) \approx_{a}^{\mathfrak{M}} \operatorname{rel}\left(h_{2}\right)$; and from $h_{2} \sim_{a}^{T_{s}} h_{3}$ that $\operatorname{ref}\left(h_{3}\right) \sim_{a}^{T_{s}} h_{2}$ and lastr $\left(\operatorname{ref}\left(h_{3}\right)\right) \sim \sim_{a}^{\mathfrak{M}} \operatorname{rel}\left(h_{3}\right)$. Furthermore, because $r e f\left(h_{3}\right) \in \Delta^{i}$ we can deduce from the transitivity of $\sim_{a}^{T_{s}}$ (and by induction) that $\operatorname{ref}\left(h_{3}\right) \sim_{a}^{T_{s}} h_{1}$ and hence $h_{3} \sim_{a}^{T_{s}} h_{1}$ by definition.

$h_{1} \in \Delta^{i}, h_{2}, h_{3} \in \Delta^{i+1}$ : We have that $\operatorname{ref}\left(h_{2}\right) \sim_{a}^{T_{s}} h_{1}$ and $\operatorname{lastr}\left(\operatorname{ref}\left(h_{2}\right)\right) \sim_{a}^{T_{s}} \operatorname{rel}\left(h_{2}\right)$ and jump $\left(h_{2}\right)=a$. Then, we also have that $\operatorname{rel}\left(h_{2}\right) \approx_{a}^{T_{s}} \operatorname{rel}\left(h_{3}\right)$ and jump $\left(h_{3}\right)=$ jump $\left(h_{2}\right)=a$ and $\operatorname{ref}\left(h_{2}\right) \sim_{a}^{T_{s}}$ ref $\left(h_{3}\right)$ proving that also $h_{1} \sim_{a}^{T_{s}} h_{3}$ by induction.

$h_{1}, h_{3} \in \Delta^{i}, h_{2} \in \Delta^{i+1}$ : We have that $\operatorname{ref}\left(h_{2}\right) \sim_{a}^{T_{s}} h_{1}$ and $\operatorname{lastr}\left(\operatorname{ref}\left(h_{2}\right)\right) \sim_{a}^{T_{s}} \operatorname{rel}\left(h_{2}\right)$ and jump $\left(h_{2}\right)=a$ and $\operatorname{ref}\left(h_{2}\right) \sim_{a}^{T_{s}} h_{3}$ and thus $h_{1} \sim_{a}^{T_{s}} h_{3}$ (by induction).

$h_{1}, h_{3} \in \Delta^{i+1}, h_{2} \in \Delta^{i}$ : We have that $\operatorname{ref}\left(h_{1}\right) \sim_{a}^{T_{s}} h_{2}$ and $\operatorname{lastr}\left(\operatorname{ref}\left(h_{1}\right)\right) \sim_{a}^{T_{s}} \operatorname{rel}\left(h_{1}\right)$ and jump $\left(h_{1}\right)=a$ and $\operatorname{ref}\left(h_{3}\right) \sim_{a}^{T_{s}} h_{2} \operatorname{lastr}\left(\operatorname{ref}\left(h_{3}\right)\right) \sim_{a}^{T_{s}} \operatorname{rel}\left(h_{3}\right)$ and jump $\left(h_{3}\right)=a$. But then by induction $\operatorname{ref}\left(h_{1}\right) \sim_{a}^{T_{s}} \operatorname{ref}\left(h_{3}\right)$ and $\operatorname{rel}\left(h_{1}\right) \underset{a}{\mathfrak{M}} \operatorname{rel}\left(h_{3}\right)$ which shows that $h_{1} \sim{ }_{a}^{T_{s}} h_{3}$.

Proposition 8 The $i_{S} R$-pando unfolding of a pointed $\boldsymbol{i C G S}$ is $i_{S} R$-pando-like.

Proof Let $\mathfrak{M}$ be an iCGS and $T_{S}$ its $i_{S} R$-pando unfolding. For each $h \in S t^{\prime}$ with $|\operatorname{rel}(h)|=1$ we define $S t_{h}$ as the set of states/histories in $S t^{\prime}$ reachable from $h$, i.e. $S t_{h}=\left\{h^{\prime} \in S t^{\prime} \mid\right.$ $\left.\rho_{T_{s}}\left(h, h^{\prime}\right) \neq \epsilon\right\}$. Let $\mathfrak{M}_{h}$ denote the submodel of $T_{s}$ which does only consist of states $S t_{h}$ and in which the domain of all elements is restricted to $S t_{h}$. Moreover, we take $I=\{h \in S t \mid$ $|\operatorname{rel}(h)|=1\}$.

Claim: We have that $S t^{\prime}=\biguplus_{h \in I} S t_{h}$ and each $\mathfrak{M}_{h}$ is $i_{o} R$-tree-like.

Proof of claim: Clearly, all sets $S t_{h}$ are mutually disjoint and each $h \in S t$ has to occur in some $S t_{h}$. It is also obvious that each $\mathfrak{M}_{h}$ has tree-structure. Now suppose $h_{1}, h_{2} \in S t_{h}$ with $h_{1} \sim{ }_{a}^{T_{s}} h_{2}$; then, by definition also $h_{1} \approx_{a}^{\mathfrak{M}} h_{2}$.

We proceed with the main proof and define $\hat{\sim}_{a}$ as the subset of $\sim_{a}^{T_{s}}$ which exists between sets $S t_{h}$ and $S t_{h^{\prime}}$ with $h \neq h^{\prime}$. From Lemma 8 it follows that $\sim_{a}^{T_{s}}$ is transitive and that $\hat{\sim}_{a}$ is symmetric. Moreover, by definition $\hat{\sim}_{a} \cap\left(S t_{h} \times S t_{h}\right)=\emptyset$ for all $h \in S t^{\prime}$.

The fourth condition of Definition 16 is obvious from the definition of the $i_{S} R$-pando unfolding. It remains to show the fifth condition of Definition 16. Suppose $h_{1}, h_{1}^{\prime} \in S t_{\bar{h}_{1}}$ and $h_{1}\left(\sim_{\mathbb{A g t}}\right)^{*} h_{1}^{\prime}$. Then, also $h_{1}, h_{1}^{\prime} \in \Delta_{i}$ for some $i$. From Lemma 6 we obtain that $\operatorname{rel}\left(h_{1}\right)\left(\approx_{\mathbb{A g t}}^{\mathfrak{M}}\right.$ )$^{*} \operatorname{rel}\left(h_{1}^{\prime}\right)$, i.e. that both nodes reside on the same level.

The following two lemmata are needed to prove Theorem 3. The first lemma states that the set of epistemic alternatives to any state is the same in the model and in the pando unfolding. 
Lemma 9 Let $\mathfrak{M}$ be an $\boldsymbol{i}$ CGS and $q_{0}$ a state in it. Then, the following property holds: For all $A \subseteq \mathbb{A g t}$ and all nodes $h$ in $T_{s}\left(\mathfrak{M}, q_{0}\right)$ we have that $\left\{q \mid \operatorname{lastr}(h) \sim \sim_{A}^{\mathfrak{M}} q, q \in S t_{\mathfrak{M}}\right\}=$ $\left\{\operatorname{lastr}\left(h^{\prime}\right) \mid h^{\prime} \sim_{A}^{T_{s}\left(\mathfrak{M}, q_{0}\right)} h, h^{\prime} \in \operatorname{St}_{T_{s}\left(\mathfrak{M}, q_{0}\right)}\right\}$.

Proof " $\subseteq$ ": Suppose $h=h^{\prime} q^{\prime} \in \Delta_{\mathfrak{M}}^{i}\left(q_{0}\right)$ and $q^{\prime} \sim \sim_{A}^{\mathfrak{M}} q$ and $h^{\prime} \neq \epsilon$ (the case for $h^{\prime}=\epsilon$ is clear). Then, there is some $a \in A$ with $q^{\prime} \sim_{a}^{\mathfrak{M}} q$ and thus $h^{\prime \prime}:=\hat{h} \hat{a} q \in \Delta_{\mathfrak{M}}^{i+1}\left(q_{0}\right) \subseteq$ $S t_{T_{s}\left(\mathfrak{M}, q_{0}\right)}$ with $\hat{h} \sim_{a}^{T_{s}} h$ by definition of the $\Delta_{\mathfrak{M}}^{j}$ 's. By Definition 17 also $h^{\prime \prime} \sim_{A}^{T_{s}\left(\mathfrak{M}, q_{0}\right)} h$. The claim follows as lastr $\left(h^{\prime \prime}\right)=q$.

“ $\supseteq$ ”: Suppose $h^{\prime} \sim_{A}^{T_{s}\left(\mathfrak{M}, q_{0}\right)} h$ and $h^{\prime} \in S t_{T_{s}\left(\mathfrak{M}, q_{0}\right)}$. The claim is clear if $h, h^{\prime} \in \Delta_{\mathfrak{M}}^{i}\left(q_{0}\right)$. According to Definition 17 the remaining case is when $h \in \Delta_{\mathfrak{M}}^{i}(q)$ and $h^{\prime} \in \Delta_{\mathfrak{M}}^{i+1}(q)$, or the roles of $h$ and $h^{\prime}$ switched. Then, $h^{\prime}=\hat{h} \hat{a} q, \hat{h} \sim_{a}^{T_{s}} h$ for some $a \in \mathbb{A g t}$ and $\operatorname{lastr}(\hat{h}) \sim_{a}^{\mathfrak{M}} q$. The claim follows as lastr $\left(h^{\prime}\right)=q$ and $\operatorname{lastr}(h) \underset{A}{\mathfrak{M}} q$.

The next lemma is needed to show that the witnessing strategy which we shall construct in the invariance Theorem 3 is uniform.

Lemma 10 Let $\mathfrak{M}$ be an iCGS, $q$ a state in it, $T_{s}=T_{s}(\mathfrak{M}, q), h, \hat{h}_{1}, \hat{h}_{2} \in S t_{T_{s}}, A \subseteq \mathbb{A g t}$, and $a \in \mathbb{A g t}$. If $\hat{h}_{1} \sim_{A}^{T_{s}} h \sim_{A}^{T_{s}} \hat{h}_{2}$ and $h_{1}=\hat{h}_{1} h_{1}^{F} \sim_{a} \hat{h}_{2} h_{2}^{F}=h_{2}$ with $h_{1}^{F}, h_{2}^{F} \in \Lambda^{\text {fin }}$; then, $\left(\operatorname{rel}\left(h_{1}\right) \approx_{a}^{\mathfrak{M}} \operatorname{rel}\left(h_{2}\right), \rho\left(\hat{h}_{1}, h_{1}\right) \approx_{a}^{\mathfrak{M}} \rho\left(\hat{h}_{2}, h_{2}\right)\right.$ and $\left.\left|h_{1}^{F}\right|=\left|h_{2}^{F}\right|\right)$, or $h_{1}^{F}=h_{2}^{F}=\epsilon$.

Proof Suppose $\hat{h}_{1} \sim_{b}^{T_{s}} h \sim_{c}^{T_{s}} \hat{h}_{2}$ with $b, c \in A$. From Lemma 3 we obtain $\rho\left(\hat{h}_{1}, h_{1}\right) \approx_{a}^{\mathfrak{M}}$ $\rho\left(\hat{h}_{2}, h_{2}\right)$ by taking $\hat{q}_{i}=\hat{h}_{i}$ and $q_{i}=h_{i}$ and $q=h$ for $i=1,2$.

To prove the lemma, we firstly assume that $h_{1}, h_{2} \in \Delta^{i}$ for some $i \in \mathbb{N}_{0}$. Then, by definition $\operatorname{rel}\left(h_{1}\right) \underset{a}{\mathfrak{M}} \operatorname{rel}\left(h_{2}\right)$ and the claim follows.

Secondly, suppose w.l.o.g. $h_{1} \in \Delta^{i}$ and $h_{2} \in \Delta^{i+1}$ for some $i \in \mathbb{N}_{0}$. In this case $\operatorname{rel}\left(h_{2}\right) \in$ St and thus $h_{2}^{F}=\epsilon$ and $\rho\left(\hat{h}_{2}, h_{2}\right)=\operatorname{rel}\left(h_{2}\right) \approx_{a}^{\mathfrak{M}} \rho\left(\hat{h}_{1}, h_{1}\right)$. The latter implies that $\left|\rho\left(\hat{h}_{1}, h_{1}\right)\right|=1$ and hence $h_{1}^{F}=\epsilon$.

Theorem 3 For every $i$ CGS $\mathfrak{M}$, state $q$ in $\mathfrak{M}$, and $\mathbf{A T L}^{*}$-formula $\varphi$, it holds that

$$
\mathfrak{M}, q \models_{i_{s} R} \varphi \text { iff } T_{s}(\mathfrak{M}, q), q \models_{i_{s} R} \varphi \text { iff } T_{s}(\mathfrak{M}, q), q \models_{i_{s} r} \varphi .
$$

Proof We show that for every node $h$ in $T_{s}:=T_{S}(\mathfrak{M}, q)$ it holds that $\mathfrak{M}$, lastr $(h) \models_{i_{S} R} \varphi$ iff $T_{s}(\mathfrak{M}, q), h \models_{i_{s} r} \varphi$. Then, the claim follows from Propositions 7 and 8 for $h=q$. The proof is done by induction on the structure of $\varphi$ and is similar to the proof given for Theorem 2 .

Base cases:

Propositional case: Straightforward.

Case: $\varphi \equiv\langle\langle A\rangle\rangle \gamma$ where $\gamma$ contains no nested strategic modalities.

" $\Rightarrow$ ": Suppose we have $\mathfrak{M}$, lastr $(h) \models_{i_{S} R}\left\langle\langle A\rangle \gamma\right.$ and let $s_{A}$ be an $i R$-strategy with

$$
(\star) \forall \lambda \in \operatorname{out}_{\mathfrak{M}}^{i_{s}}\left(\operatorname{lastr}(h), s_{A}\right): \mathfrak{M}, \lambda \models_{i_{S} R} \gamma .
$$

We construct the $i r$-strategy $s_{A}^{\prime}$ in $T_{S}(\mathfrak{M}, q)$ as follows: for all $\hat{h} \in S t_{T_{S}}$ with $h \sim_{A}^{T_{s}} \hat{h}$ and all $h^{F} \in \Lambda_{\mathfrak{M}}^{\text {fin }}(\operatorname{lastr}(\hat{h}))$ we set

$$
s_{a}^{\prime}\left(\hat{h}\left(h^{F}[1, \infty]\right)\right):=s_{a}\left(h^{F}\right) .
$$

We note that we have to exclude the first state in $h^{F}$ because it is already contained in $\hat{h}$. For all other histories $h^{\prime \prime}$ (which do not have the prescribed form) we define $s_{a}^{\prime}\left(h^{\prime \prime}\right)$ arbitrarily but in a uniform way. The setting is illustrated in Fig. 16. 
Fig. 16 Setting of the proof of Theorem 3

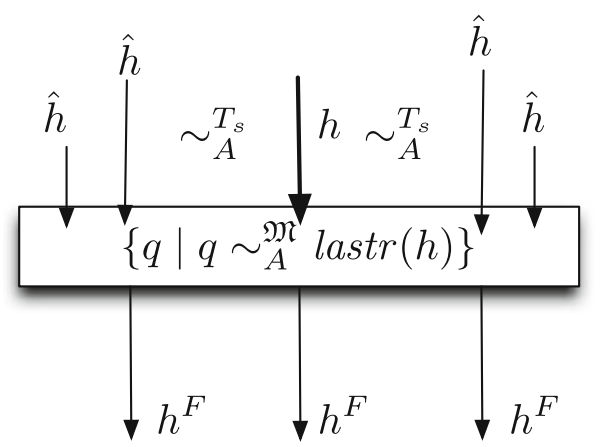

Clearly, we have that each $\hat{h}\left(h^{F}[1, \infty]\right)$ is a valid state in $T_{s}$ and by Lemma $10, s_{a}^{\prime}$ is well-defined: Suppose, there are $h_{1}=\hat{h}_{1} h_{1}^{F}$ and $h_{2}=\hat{h}_{2} h_{2}^{F}$ with $h_{1}=h_{2}$. Then, also $h_{1} \sim_{a}^{T_{s}} h_{2}$ and by Lemma $10 h_{1}^{F}=h_{2}^{F}$ which shows that $s_{a}^{\prime}\left(h_{1}\right)=s_{a}^{\prime}\left(h_{2}\right)$.

In the following we show that $s_{a}^{\prime}$ is uniform. Let $h_{1}$ and $h_{2}$ be two histories with $h_{1} \sim_{a}^{T_{s}} h_{2}$.

1. Assume that both nodes have the form from above, i.e. $h_{1}=\hat{h}_{1} h_{1}^{F}, h_{1}=\hat{h}_{1} h_{1}^{F}$ and $h_{1} \sim_{A}^{T_{s}} h \sim_{A}^{T_{s}} h_{2}$. Then, uniformity follows from Lemma 10.

2. Choices for two histories $h_{1}$ and $h_{2}$ where at least one does not have the required form can be defined in a uniform way by definition because $\sim_{a}^{T_{s}}$ is an equivalence relation.

Finally, we show that the sets of outcome paths are isomorphic wrt. both strategies. By Lemma 9 we have that for all states $q_{0}$ in $\mathfrak{M}$ the following holds $q_{0} \sim_{A}^{\mathfrak{M}} \operatorname{lastr}(h)$ iff there is a history $h^{\prime}$ with $h^{\prime} \sim_{A}^{T_{S}} h$ and $\operatorname{lastr}\left(h^{\prime}\right)=q_{0}$. We denote one of these histories $h^{\prime}$ by $h\left(q_{0}\right)$. Then, by construction of $s_{A}^{\prime}$ we have that

$$
\begin{aligned}
& q_{0} q_{1} q_{2} \cdots \in \operatorname{out}_{\mathfrak{M}}^{i_{s}}\left(\operatorname{last}(h), s_{A}\right) \\
& \quad \text { iff }\left(h^{\prime}\right)\left(h^{\prime} q_{1}\right)\left(h^{\prime} q_{1} q_{2}\right) \cdots \in \operatorname{out}_{T_{s}(\mathfrak{M}, q)}^{i_{s}}\left(h, s_{A}^{\prime}\right) \\
& \quad \text { for some } h^{\prime}=h\left(q_{0}\right) .
\end{aligned}
$$

Since the valuation of propositions does only depend on the final state of a history and by $(\star)$ we have $T_{s}(\mathfrak{M}, q), h \models_{i_{s} r}\langle\langle A\rangle\rangle \gamma$.

$\Leftarrow$ : For the other direction, suppose we have $T_{S}(\mathfrak{M}, q), h \models_{i_{s} r}\langle\langle A\rangle\rangle \gamma$. So, there is an $i r$-strategy $s_{A}$ such that

$$
(\star) \forall \lambda \in \operatorname{out}_{T_{s}(\mathfrak{M}, q)}^{i_{s}}\left(h, s_{A}\right): T_{s}(\mathfrak{M}, q), \lambda \models i_{s} r \gamma .
$$

We construct a witnessing $i R$-strategy $s_{A}^{\prime}$ in $\mathfrak{M}$ as follows: $s_{a}^{\prime}\left(h^{F}\right)=s_{a}\left(\hat{h} \hat{a} h^{F}\right)$ for every $a \in A, \hat{h} \sim_{A}^{T_{S}} h$ for $h^{F} \in \Lambda^{f i n}\left(q^{\prime}\right)$ with $q^{\prime} \sim_{A}^{\mathfrak{M}} \operatorname{lastr}(h)$ and arbitrary but in a uniform way for all other histories. It is easy to verify that each strategy $s_{a}^{\prime}$ is uniform and welldefined. Moreover, $s_{A}^{\prime}$ yields an equivalent (apart from the notational differences) set of outcome paths as above. We have $\mathfrak{M}, \operatorname{lastr}(h) \models_{i_{s} R}\langle\langle A\rangle \gamma$.

Induction step:

Case: $\varphi \equiv \psi_{1} \wedge \psi_{2}$. Straightforward.

Case: $\varphi \equiv \neg \psi$. M , lastr $(h) \models_{i_{s} R} \neg \psi$ iff not $\mathfrak{M}$, lastr $(h) \models_{i_{S} R} \psi$ iff (by induction hypothesis) not $T_{S}(\mathfrak{M}, q), h \models_{i_{s} R} \psi$ iff $T_{S}(\mathfrak{M}, q), h \models_{i_{S} R} \neg \psi$. 
Case: $\varphi \equiv\left\langle\langle A\rangle \gamma\right.$. By induction hypothesis we have for each history $h$ in $T_{s}(\mathfrak{M}, q)$ and each strict state-subformula $\varphi^{\prime}$ of $\gamma$ that $\mathfrak{M}, \operatorname{lastr}(h) \models_{i_{s} R} \varphi^{\prime}$ iff $T_{s}(\mathfrak{M}, q), h \models_{i_{s} r} \varphi^{\prime}$. For any maximal strict state-subformula $\varphi^{\prime}$ we label all states $h$ in $T_{s}(\mathfrak{M}, q)$ and states lastr $(h)$ in $\mathfrak{M}$ with a new proposition $\mathbf{p}_{\varphi^{\prime}}$ iff $\varphi^{\prime}$ holds in this very state. Then, we replace each $\varphi^{\prime}$ in $\varphi$ with proposition $\mathbf{p}_{\varphi^{\prime}}$ and the claim follows by induction.

\section{References}

1. Ågotnes, T. (2004). A note on syntactic characterization of incomplete information in ATEL. In Proceedings of the workshop on knowledge and games, Liverpool (pp. 34-42).

2. Ågotnes, T. (2006). Action and knowledge in alternating-time temporal logic. Synthese, 149(2), 377-409.

3. Ågotnes, T., Goranko, V., \& Jamroga, W. (2007). Alternating-time temporal logics with irrevocable strategies. In D. Samet (Ed.), Proceedings of TARK XI (pp. 15-24).

4. Ågotnes, T., \& Walther, D. (2009). A logic of strategic ability under bounded memory. Journal of Logic, Language and Information, 18(1), 55-77.

5. Alechina, N., Logan, B., Nga, N. H., \& Rakib, A. (2009). A logic for coalitions with bounded resources. In Proceedings of IJCAI (pp. 659-664).

6. Alechina, N., Logan, B., Nga, N. H., \& Rakib, A. (2010). Resource-bounded alternating-time temporal logic. In Proceedings of AAMAS (pp. 481-488).

7. Alur, R., Henzinger, T. A., \& Kupferman, O. (1997). Alternating-time temporal logic. In Proceedings of the 38th annual symposium on foundations of computer science (FOCS) (pp. 100-109). Los Alamitos: IEEE Computer Society Press.

8. Alur, R., Henzinger, T. A., \& Kupferman, O. (1998). Alternating-time temporal logic. Lecture Notes in Computer Science, 1536, 23-60.

9. Alur, R., Henzinger, T. A., \& Kupferman, O. (2002). Alternating-time temporal logic. Journal of the ACM, 49, 672-713.

10. Alur, R., Henzinger, T. A., Kupferman, O., \& Vardi, M. Y. (1998). Alternating refinement relations. In Proceedings of CONCUR, volume 1466 of LNCS (pp. 163-178).

11. Brihaye, T., Da Costa Lopes, A., Laroussinie, F., \& Markey, N. (2009). ATL with strategy contexts and bounded memory. In Proceedings of LFCS, volume 5407 of Lecture notes in computer science (pp. 92-106). New York: Springer.

12. Bulling, N., \& Dix, J. (2010). Modelling and verifying coalitions using argumentation and ATL. Inteligencia Artificial, Revista Iberoamericana de Inteligencia Artificial, 14(46), 45-73.

13. Bulling, N., Dix, J., \& Jamroga, W. (2010). Model checking logics of strategic ability: Complexity. In M. Dastani, K. Hindriks, \& J.-J. Meyer (Eds.), Specification and verification of multi-agent systems (pp. $125-159)$. New York: Springer.

14. Bulling, N., \& Farwer, B. (2009). Expressing properties of resource-bounded systems: The logics RTL* and RTL. In Proceedings of CLIMA (pp. 22-45).

15. Bulling, N., \& Farwer, B. (2010). On the (un-)decidability of model checking resource-bounded agents. In Proceedings of ECAI, volume 215 of Frontiers in artificial intelligence and applications (pp. 567-572). Amsterdam: IOS Press.

16. Bulling, N., \& Jamroga, W. (2009). What agents can probably enforce. Fundamenta Informaticae, 93(13), 81-96.

17. Bulling, N., \& Jamroga, W. (2010). Verifying agents with memory is harder than it seemed. AI Соттиnications, 23, 380-403.

18. Bulling, N., \& Jamroga, W. (2011). Alternating epistemic mu-calculus. In Proceedings of IJCAI-11 (pp. 109-114).

19. Bulling, N., Jamroga, W., \& Dix, J. (2008). Reasoning about temporal properties of rational play. Annals of Mathematics and Artificial Intelligence, 53(1-4), 51-114.

20. Chatterjee, K., Henzinger, T. A., \& Piterman, N. (2007). Strategy logic. In Proceedings of CONCUR (pp. 59-73).

21. Clarke, E. M., \& Emerson, E. A. (1981). Design and synthesis of synchronization skeletons using branching time temporal logic. In Proceedings of logics of programs workshop, volume 131 of Lecture notes in computer science (pp. 52-71).

22. Davis, E. (1994). Knowledge preconditions for plans. Journal of Logic and Computation, 4(5), 721-766. 
23. Dima, C., Enea, C., \& Guelev, D. P. (2010). Model-checking an alternating-time temporal logic with knowledge, imperfect information, perfect recall and communicating coalitions. In Proceedings of GANDALF (pp. 103-117).

24. Dima, C., \& Tiplea, F. L. (2011). Model-checking atl under imperfect information and perfect recall semantics is undecidable. CoRR, abs/1102.4225.

25. Ghaderi, H., Levesque, H., \& Lespérance, Y. (2007). A logical theory of coordination and joint ability. In Proceedings of AAAI'07 (pp. 421-426). Menlo Park, CA: AAAI Press.

26. Goranko, V. (2001). Coalition games and alternating temporal logics. In J. van Benthem (Ed.), Proceedings of TARK VIII (pp. 259-272). San Francisco: Morgan Kaufmann.

27. Goranko, V., \& Jamroga, W. (2004). Comparing semantics of logics for multi-agent systems. Synthese, 139(2), 241-280.

28. Goranko, V., Jamroga, W., \& Turrini, P. (2011). Strategic games and truly playable effectivity functions. In Proceedings of AAMAS2011 (pp. 727-734).

29. Goranko, V., Jamroga, W., \& Turrini, P. (2013). Strategic games and truly playable effectivity functions. Journal of Autonomous Agents and Multi-Agent systems, 26(2), 288-314.

30. Goranko, V., \& Shkatov, D. (2009). Tableau-based decision procedures for logics of strategic ability in multiagent systems. ACM Transactions on Computational Logic, 11(1), 1-48.

31. Goranko, V., \& van Drimmelen, G. (2006). Complete axiomatization and decidability of alternating-time temporal logic. Theoretical Computer Science, 353(1), 93-117.

32. Guelev, D. P., Dima, C., \& Enea, C. (2011). An alternating-time temporal logic with knowledge, perfect recall and past: Axiomatisation and model-checking. Journal of Applied Non-Classical Logics, 21(1), 93-131.

33. Harding, A., Ryan, M., \& Schobbens, P.-Y. (2002). Approximating ATL* in ATL. In VMCAI '02: Revised papers from the third international workshop on verification, model checking, and abstract interpretation (pp. 289-301). New York: Springer.

34. Hawke, P. (2010). Coordination, almost perfect information and strategic ability. In Proceedings of LAMAS.

35. Jamroga, W. (2003). Some remarks on alternating temporal epistemic logic. In B. Dunin-Keplicz \& R. Verbrugge (Eds.), Proceedings offormal approaches to multi-agent systems (FAMAS 2003) (pp. 133-140).

36. Jamroga, W. (2006). On the relationship between playing rationally and knowing how to play: A logical account. In C. Freksa, M. Kohlhase, \& K. Schill (Eds.), Proceedings of KI 2006, volume 4314 of Lecture notes in artificial intelligence (pp. 419-433). New York: Springer.

37. Jamroga, W. (2008). A temporal logic for stochastic multi-agent systems. In Proceedings of PRIMA'08, volume 5357 of LNCS (pp. 239-250).

38. Jamroga, W., \& Ågotnes, T. (2006). Modular interpreted systems: A preliminary report. Technical report IfI-06-15, Clausthal University of Technology.

39. Jamroga, W., \& Ågotnes, T. (2007). Constructive knowledge: What agents can achieve under incomplete information. Journal of Applied Non-Classical Logics, 17(4), 423-475.

40. Jamroga, W., \& Bulling, N. (2010). Comparing variants of strategic ability. In Proceedings of EUMAS2010.

41. Jamroga, W., \& Bulling, N. (2011). Comparing variants of strategic ability. In Proceedings of IJCAI-11 (pp. 252-257).

42. Jamroga, W., \& Dix, J. (2008). Model checking abilities of agents: A closer look. Theory of Computing Systems, 42(3), 366-410.

43. Jamroga, W., \& van der Hoek, W. (2004). Agents that know how to play. Fundamenta Informaticae, 63(2-3), 185-219.

44. Jamroga, W., van der Hoek, W., \& Wooldridge, M. (2005). Intentions and strategies in game-like scenarios. In C. Bento, A. Cardoso, \& G. Dias (Eds.), Progress in artificial intelligence: Proceedings of EPIA 2005, volume 3808 of Lecture notes in artificial intelligence (pp. 512-523). New York: Springer.

45. Jonker, G. (2003). Feasible strategies in alternating-time temporal epistemic logic. Master thesis, University of Utrecht.

46. Kacprzak, M., \& Penczek, W. (2004). Unbounded model checking for alternating-time temporal logic. In Proceedings of AAMAS-04.

47. Köster, M., \& Lohmann, P. (2011). Abstraction for model checking modular interpreted systems over ATL. In Proceedings of AAMAS (pp. 1129-1130).

48. Laroussinie, F., Markey, N., \& Oreiby, G. (2008). On the expressiveness and complexity of ATL. Logical Methods in Computer Science, 4, 7.

49. Lesperance, Y., Levesque, H. J., Lin, F., \& Scherl, R. B. (2000). Ability and knowing how in the situation calculus. Studia Logica, 66(1), 165-186. 
50. Mogavero, F., Murano, A., Perelli, G., \& Vardi, M. Y. (2012). What makes ATL* decidable? A decidable fragment of strategy logic. In Proceedings of CONCUR (pp. 193-208).

51. Mogavero, F., Murano, A., \& Vardi, M. Y. (2010). Reasoning about strategies. In Proceedings of FSTTCS (pp. 133-144).

52. Pauly, M. (2001). Logic for social software. PhD thesis, University of Amsterdam.

53. Pauly, M. (2002). A modal logic for coalitional power in games. Journal of Logic and Computation, 12(1), 149-166.

54. Schewe, S. (2008). ATL* satisfiability is 2ExpTime-complete. In Proceedings of ICALP 2008, volume 5126 of Lecture notes in computer science (pp. 373-385). New York: Springer.

55. Schnoor, H. (2010). Deciding epistemic and strategic properties of cryptographic protocols. Technical report tr_1012, Kiel University.

56. Schnoor, H. (2010). Strategic planning for probabilistic games with incomplete information. In Proceedings of AAMAS'10 (pp. 1057-1064).

57. Schobbens, P. Y. (2004). Alternating-time logic with imperfect recall. Electronic Notes in Theoretical Computer Science, 85(2), 82-93.

58. van der Hoek, W., Jamroga, W., \& Wooldridge, M. (2005). A logic for strategic reasoning. In Proceedings of AAMAS'05 (pp. 157-164).

59. van der Hoek, W., Lomuscio, A., \& Wooldridge, M. (2006). On the complexity of practical ATL model checking. In P. Stone \& G. Weiss (Eds.), Proceedings of AAMAS'06 (pp. 201-208).

60. van der Hoek, W., \& Wooldridge, M. (2002). Tractable multiagent planning for epistemic goals. In C. Castelfranchi \& W. L. Johnson (Eds.), Proceedings of the first international joint conference on autonomous agents and multi-agent systems (AAMAS-02) (pp. 1167-1174). New York: ACM Press.

61. van der Hoek, W., \& Wooldridge, M. (2003). Cooperation, knowledge and time: Alternating-time temporal epistemic logic and its applications. Studia Logica, 75(1), 125-157.

62. van Otterloo, S., \& Jonker, G. (2004). On epistemic temporal strategic logic. Electronic Notes in Theoretical Computer Science, XX, 35-45. Proceedings of LCMAS'04.

63. van Otterloo, S., van der Hoek, W., \& Wooldridge, M. (2004). Preferences in game logics. In Proceedings of AAMAS-04 (pp. 152-159).

64. Walther, D., Lutz, C., Wolter, F., \& Wooldridge, M. (2006). ATL satisfiability is indeed EXPTIMEcomplete. Journal of Logic and Computation, 16(6), 765-787.

65. Walther, D., van der Hoek, W., \& Wooldridge, M. (2007). Alternating-time temporal logic with explicit strategies. In D. Samet (Ed.), Proceedings TARK XI (pp. 269-278). Leuven: Presses Universitaires de Louvain.

66. Yadav, N., \& Sardiña, S. (2012). Reasoning about agent programs using ATL-like logics. In Proceedings of JELIA (pp. 437-449). 